\title{
CHARACTERIZATION OF IN-SITU STRESS AND PERMEABILITY IN FRACTURED RESERVOIRS
}

\author{
SEMI-ANNUAL TECHNICAL PROGRESS REPORT
}

for Period 22 May-21 November 2002

\author{
by \\ Daniel R. Burns \\ and \\ M. Nafi Toksöz \\ Earth Resources Laboratory \\ Department of Earth, Atmospheric, and Planetary Sciences \\ Massachusetts Institute of Technology \\ Cambridge, MA 02139
}

December 31, 2002

\author{
Prepared for \\ THE U.S. DEPARTMENT OF ENERGY \\ AWARD NUMBER DE-FC26-02NT15346
}

This technical progress report was prepared with the support of the U.S. Department of Energy, under Award No. DE-FC26-02NT15346. However, any opinions, findings, conclusions, or recommendations expressed herein are those of the authors and do not necessarily reflect the views of the DOE. 


\section{DISCLAIMER}

This report was prepared as an account of work sponsored by an agency of the United States Government. Neither the United States Government nor any agency thereof, nor any of their employees, makes any warranty, express or implied, or assumes any legal liability or responsibility for the accuracy, completeness, or usefulness of any information, apparatus, produce, or process disclosed, or represents that its use would not infringe privately owned rights. Reference herein to any specific commercial product, process, or service by trade name, trademark, manufacturer, or otherwise does not necessarily constitute or imply its endorsement, recommendation, or favoring by the United States Government or any agency thereof. The views and opinions of authors expressed herein do not necessarily state or reflect those of the United States Government or any agency thereof. 


\begin{abstract}
We have extended a three-dimensional finite difference elastic wave propagation model previously developed at the Massachusetts Institute of Technology (MIT) Earth Resources Laboratory (ERL) for modeling and analyzing the effect of fractures on seismic waves. The code has been translated into $\mathrm{C}$ language and parallelized [using message passing interface (MPI)] to allow for larger models to be run on Linux PC computer clusters. We have also obtained another 3-D code from Lawrence Berkeley Laboratory, which we will use for verification of our ERL code results and also to run discrete fracture models. Testing of both codes is underway.

We are working on a new finite difference model of borehole wave propagation for stressed formations. This code includes coordinate stretching to provide stable, variable grid sizes that will allow us to model the thin fluid annulus layers in borehole problems, especially for acoustic logging while drilling (LWD) applications. We are also extending our analysis routines for the inversion of flexural wave dispersion measurements for insitu stress estimates. Initial results on synthetic and limited field data are promising for a method to invert cross dipole data for the rotation angle and stress state simultaneously.

A meeting is being scheduled between MIT and Shell Oil Company scientists to look at data from a fractured carbonate reservoir that may be made available to the project. The Focus/Disco seismic processing system from Paradigm Geophysical has been installed at ERL for field data analysis and as a platform for new analysis modules.

We have begun to evaluate the flow properties of discrete fracture distributions through a simple 2D numerical model. Initial results illustrate how fluid flow pathways are very sensitive to variations in the geometry and apertures of fracture network.
\end{abstract}




\section{TABLE OF CONTENTS}

$\begin{array}{ll}\text { Title Page } & 1\end{array}$

$\begin{array}{ll}\text { Disclaimer } & 2\end{array}$

$\begin{array}{ll}\text { Abstract } & 3\end{array}$

Table of Contents 4

Executive Summary 5

$\begin{array}{ll}\text { Introduction } & 6\end{array}$

$\begin{array}{ll}\text { Results and Discussion } & 7\end{array}$

$\begin{array}{ll}\text { Numerical Modeling } & 7\end{array}$

$\begin{array}{ll}\text { Inversion } & 11\end{array}$

$\begin{array}{lr}\text { Field Data } & 12\end{array}$

$\begin{array}{lr}\text { Fracture Permeability } & 12\end{array}$

$\begin{array}{ll}\text { Conclusions } & 12\end{array}$

$\begin{array}{ll}\text { References } & 13\end{array}$

Appendices

A. A Stretched Grid Finite Difference Time-Domain Scheme Implemented with Anisotropic Perfectly Matched Layer, by Huang, X., Zheng, Y., Burns, D. R., and Toksöz, M. N.

B. Simultaneous Inversion For Fast Azimuth and Dispersion of Borehole Flexure Waves Using Cross-Dipole Data, by Briggs, V., Huang, X., Rao, R., and Burns, D. R.

C. Fluid Flow Simulation in Fractured Reservoirs, by Sarkar, S., Toksöz, M. N., and Burns, D. R. 


\section{EXECUTIVE SUMMARY}

The purpose of this project is to develop and implement large-scale numerical models to quantify the effects of fracture parameter variations on seismic reflection signals and in-situ stress variations on flexural modes in boreholes. These models will be used as the basis of data analysis and inversion routines for estimating the heterogeneous fracture distribution in fractured reservoirs from seismic and borehole field data provided by Shell Oil Company. Fracture property distributions will be used to estimate the permeability tensor in the reservoir for input to reservoir simulators. During the first six months of the project we have focused on the development and testing of numerical models of elastic wave propagation and flow visualization in fracture networks.

1) In the area of seismic modeling, we have extended and developed 3-D finite difference numerical modeling codes to include elastic anisotropy and velocity heterogeneity in order to model the effects of spatial variations in fracture density. Code has been translated into $\mathrm{C}$ and parallelized [using message passing interface (MPI)] to allow for larger models to be run on Linux PC computer clusters. We have also obtained another 3-D code from Lawrence Berkeley Laboratory, which we will use for verification of our Earth Resources Laboratory (ERL) code results and also to run discrete fracture models. Testing of both codes is underway.

2) In the area of borehole acoustic logging modeling and in-situ stress estimation, we have developed a numerical modeling code to predict the effect on propagating modes of stress concentrations around a borehole. This code includes coordinate stretching to provide stable, variable grid sizes that will allow us to model the thin fluid annulus layers in borehole problems, especially for acoustic logging while drilling (LWD) applications. We are also extending our analysis routines for the inversion of flexural wave dispersion measurements for in-situ stress estimates. Initial results on synthetic and limited field data are promising for a method to invert cross dipole data for the rotation angle and stress state simultaneously. 
3) We are having discussions with Shell on selecting an appropriate field data set for the project. We have also selected and set up a seismic data analysis software platform ('Focus' by Paradigm Geophysical), a commercially available software package into which we can build analysis modules for transfer to industry.

4) In the area of fluid flow modeling, we have begun to evaluate the flow properties of discrete fracture distributions through a simple numerical model that provides a means of visualizing the velocity field. Flow pathways are shown to be very sensitive to variations in the geometry and apertures of fracture network.

\section{INTRODUCTION}

The purpose of this project is to develop and implement large-scale numerical models (running on parallel Linux clusters) to quantify the effects of fracture parameter variations on seismic reflection signals and in-situ stress variations on flexural modes in boreholes. These forward modeling efforts will be used to develop data analysis and inversion routines for estimating the heterogeneous fracture distribution in fractured reservoir from seismic and borehole field data. Fracture property distributions will be used to estimate the permeability tensor in the reservoir by using statistical and discrete feature network techniques. Such estimates will then be used as inputs to a reservoir simulator (MORES and ECLIPSE) for reservoir performance prediction. History matching will provide a measure of the sensitivity and, along with well data, an estimate of the accuracy of the methods developed.

During the first six months of the project we have focused on the development and testing of numerical models of elastic wave propagation and flow visualization in fracture networks. 


\section{RESULTS AND DISCUSSION}

\section{Numerical Modeling}

3-D finite difference models of elastic wave propagation

We have extended a 3-D finite difference elastic wave propagation model previously developed at ERL. The code has been translated into $\mathrm{C}$ and parallelized (using MPI) to allow for larger models to be run on Linux PC computer clusters. Testing of the code is continuing. We have also obtained another 3-D code from Lawrence Berkeley Laboratory, which we will use for verification of our ERL code and also to run discrete fracture models. Both codes are able to handle elastic anisotropy, use a staggered grid formulation, and have accuracy of $2^{\text {nd }}$ order in time and $4^{\text {th }}$ order in space.

The ERL code is an extension of the code originally developed in Cheng (1994). Shen et al. (2002) used a version of the code to model fractured reservoir seismic responses. The code represents fractured media by an equivalent anisotropic formation, and also allows for spatial heterogeneity in the elastic properties of the medium. We are adapting the code to include anelasticity.

To enhance the usefulness of the code, a graphic user interface (GUI) has been developed to generate the input files, visualize the geometry of the model and recording array, and test for stability. The package also contains some basic data plotting and processing steps, which will be developed further in the future. With this code, users will be able to build models, including fracture sets, and investigate the validity of various seismic indicators such as amplitude variation with offset (AVO), frequency variation with offset (FVO), and other attenuation and scattering effects. Figure 1 compares traces calculated with the ERL finite difference code to those obtained with the discrete wavenumber method (Bouchon and Aki, 1977) for a simple 2-D problem, illustrating the accuracy of the finite difference result. 


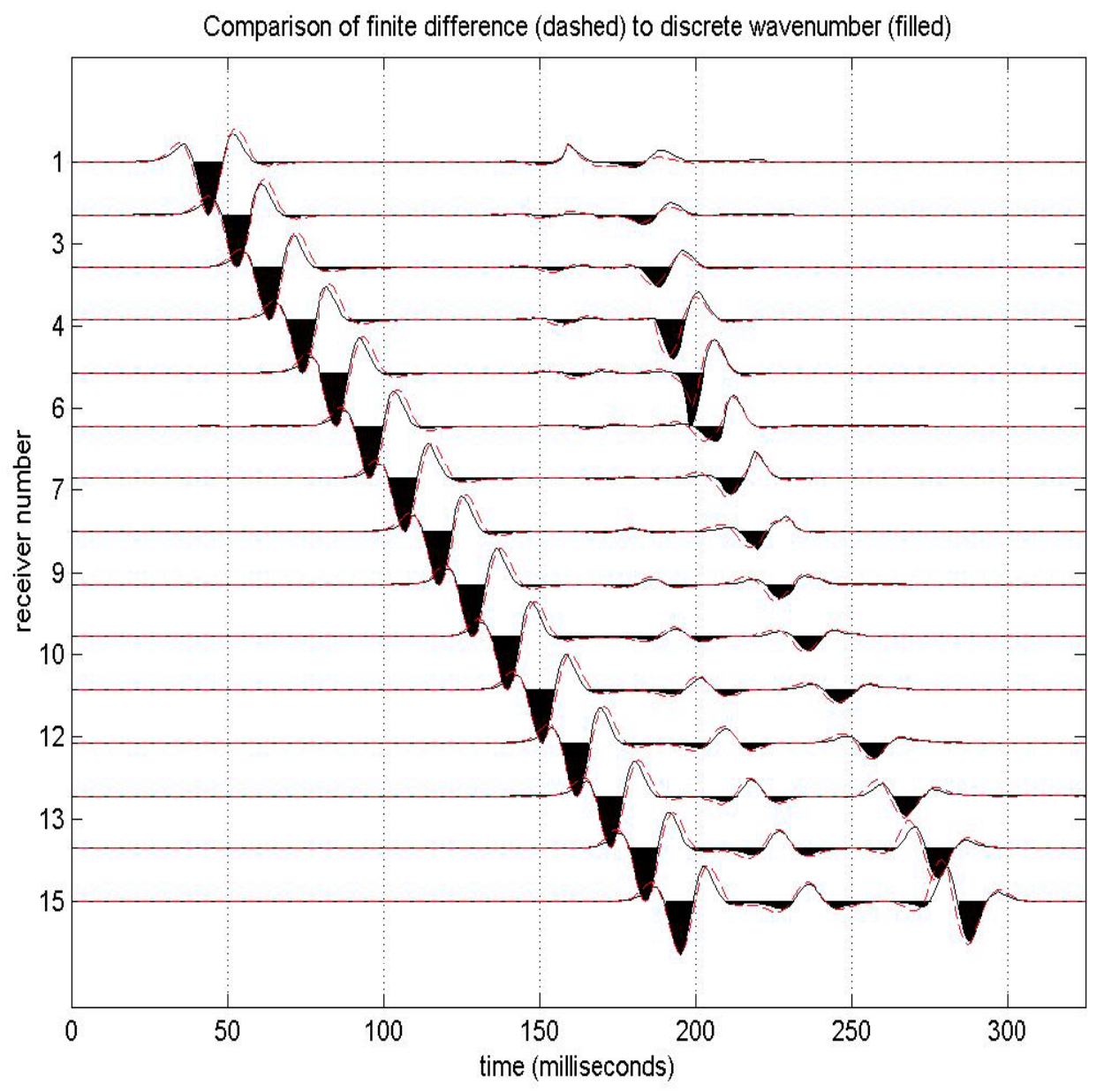

Figure 1. A comparison of finite difference (dashed line) and discrete wavenumber (solid line) numerical modeling results for a 2-D model with layered geometry. The discrete wavenumber method provides and 'exact' representation of the integral solution, so the results can be considered to be the 'exact' solution in this case. This plot provides verification of the accuracy of our finite difference results. The model has a single reflector at 300 meters depth. The source is at a depth of $50 \mathrm{~m}$ with receivers on the surface at 100 $\mathrm{m}$ spacing. The medium properties are: top layer $\mathrm{Vp}=4500 \mathrm{~m} / \mathrm{s}, \mathrm{Vs}=3000 \mathrm{~m} / \mathrm{s}$, and density $=2.2 \mathrm{~g} / \mathrm{cc}$; bottom layer $\mathrm{Vp}=5000 \mathrm{~m} / \mathrm{s}, \mathrm{Vs}=3500 \mathrm{~m} / \mathrm{s}$, and density $=2.3 \mathrm{~g} / \mathrm{cc}$.

The Lawrence Berkeley National Lab code that we recently obtained also allows for heterogeneous, anisotropic fractured reservoir models. In addition, this code allows us to model the seismic response of discrete fractures (as long as the fractures are located along one of the three planes of symmetry) using the Coates and Schoenberg (1995) thin anisotropic grid cell approach. We used this code to simulate the seismic response of two discrete fractures in a homogeneous background medium (Figure 2). The fractures extended in the $y$-direction. Figure 3 shows a snapshot of the horizontal component (Vx) of motion at 
$275 \mathrm{~ms}$ along a slice in the xz-plane at $\mathrm{y}$-coordinate $550 \mathrm{~m}$. A shot gather of the vertical component of motion $(\mathrm{Vz})$, generated using a point source with a $50 \mathrm{~Hz}$ Ricker wavelet and 11 receivers at $60 \mathrm{~m}$ interval, is shown in Figure 4. In both the snapshot and the shot gather the $\mathrm{P}$ to $\mathrm{S}$ wave conversion that occurs at the fractures is visible. We will conduct further modeling of the effects of discrete fractures to see if these seismic signatures contain information about the fracture properties, and also to see if the amplitudes are large enough to be utilized in field data.

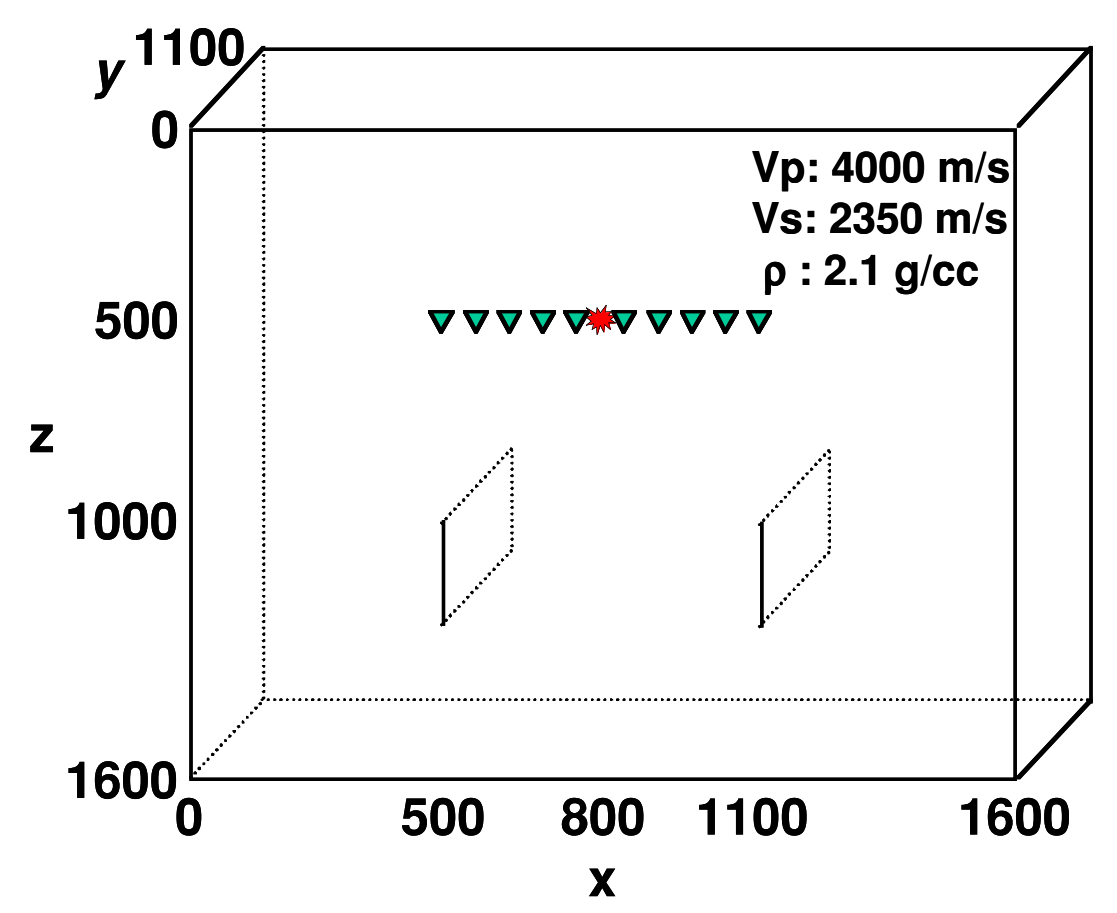

Figure 2. Geometry of the 3D finite difference model for two discrete fractures in a homogeneous background medium. 


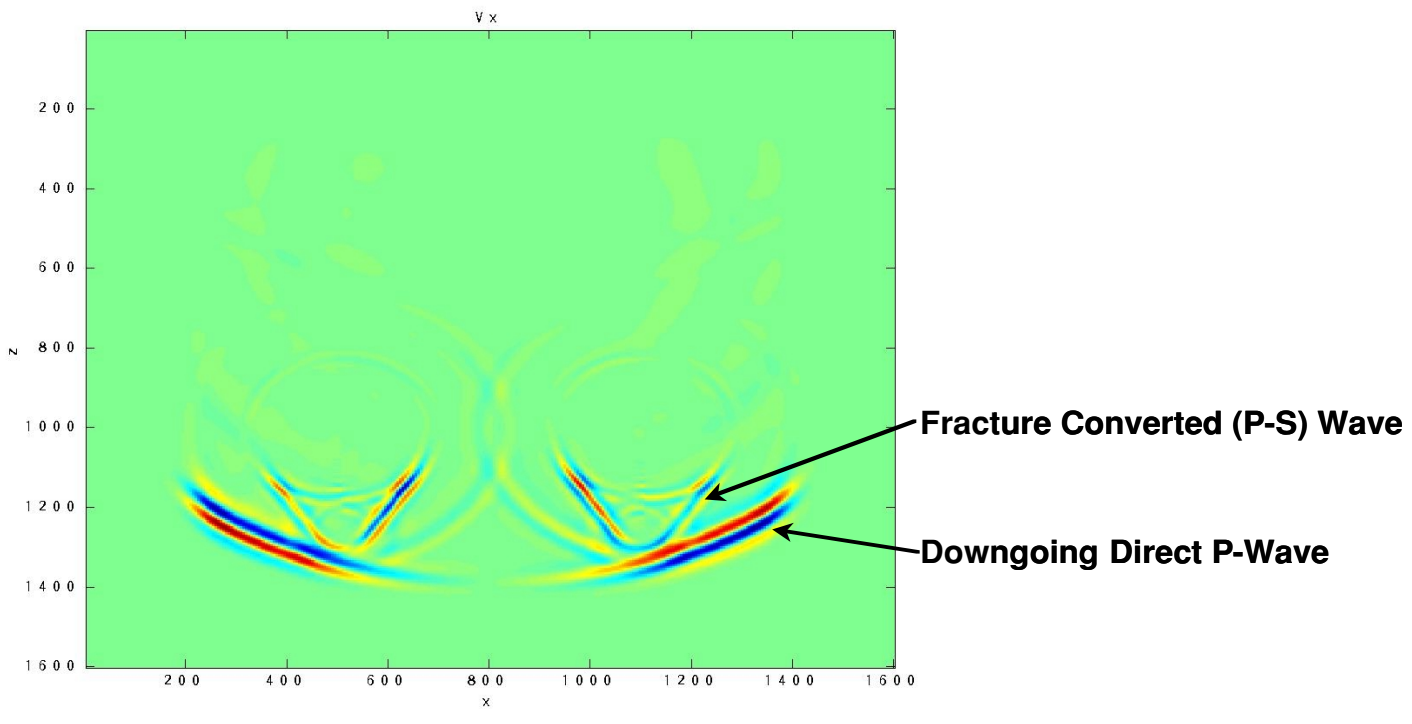

Figure 3. A snapshot of the wavefield at $\mathrm{t}=275 \mathrm{msec}$ for the horizontal motion (Vx component). The output is shown in the $\mathrm{XZ}$ plane at $\mathrm{Y}=550 \mathrm{~m}$. The downgoing direct $\mathrm{P}$ wave and the converted shear waves from the fractures are noted.

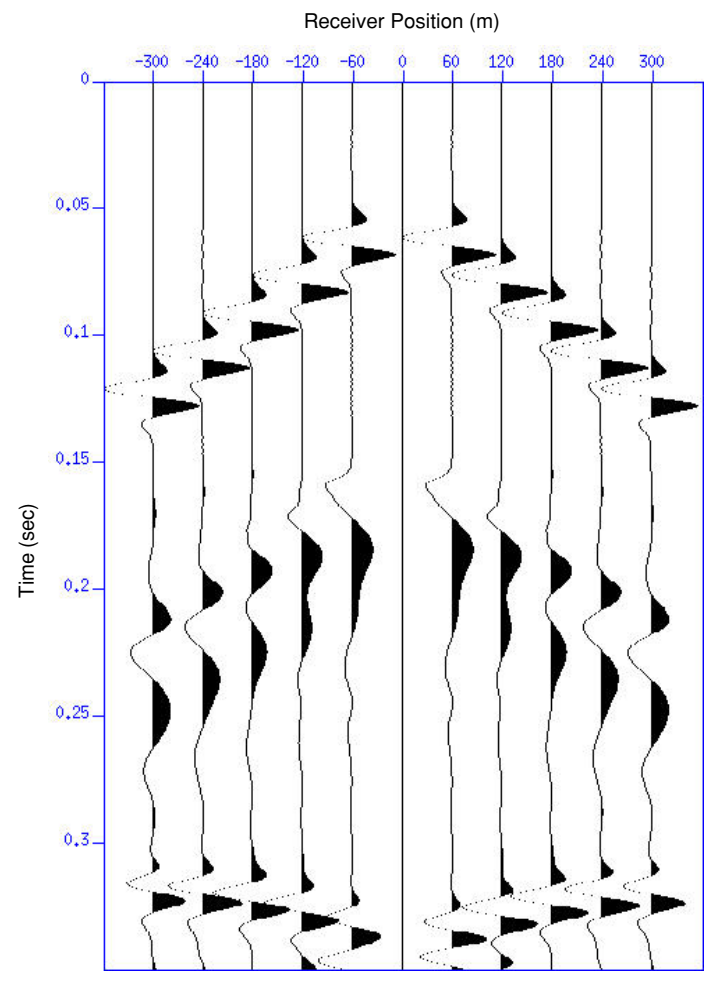

Figure 4: A shot gather (vertical component of motion $\mathrm{Vz}$ ) for the receiver line as shown in Figure 1. Automatic gain control (AGC) has been applied and the center trace was removed to allow the smaller amplitude arrivals to be seen. 
In-situ stress estimation from borehole acoustic logs

We are working on a new finite difference model of borehole wave propagation for stressed formations. This code includes coordinate stretching to provide stable, variable grid sizes that will allow us to model the thin fluid annulus layers in borehole problems, especially for acoustic logging while drilling (LWD) applications. Appendix A contains a preprint of a paper (Huang et al., 2002) describing this model development.

We are also extending our analysis routines for the inversion of flexural wave dispersion measurements for in-situ stress estimates, following the method of Huang et al. (1999). Rotation of the cross dipole acoustic logging data is a critical step for such dispersion analysis. As such, we are testing a method for inverting for the rotation angle and stress state simultaneously. Initial results with synthetic data as well as limited field data are quite promising. A preprint of a paper on this topic (Briggs et al., 2002) is given in Appendix B.

PC cluster

We recently set up a small-scale Linux PC cluster at ERL for model development and testing. The cluster consists of 12 dual CPU PC's with a total of $48 \mathrm{~Gb}$ of RAM. This cluster will allow us to test our 3-D finite difference models and our borehole models and inversion codes. For larger models we will install our code on the Shell Genesis cluster (1024 CPU's).

\section{Inversion}

Kane et al. $(1999,2001)$ have developed a method for 3-D velocity inversion from seismic data using well log constraints. These methods, which combine geostatistics and seismic inversion, provide one approach to looking at velocity heterogeneity as an indicator of fracture density variations in a reservoir. We are continuing to improve this approach and will test its applicability to the seismic field data. We will also be developing methods for extracting fracture parameters from anisotropy measurements and the seismic scattered wavefield. 


\section{Field Data}

We have had several discussions with Shell concerning the data needs for the project. A meeting is being scheduled between MIT and Shell scientists to look at data from a fractured carbonate reservoir that may be made available to the project.

A production quality seismic data processing and analysis platform has been selected and installed at ERL. The Focus/Disco seismic processing system from Paradigm Geophysical has 2-D and 3-D processing capability with DLT 7000 tape support. Eight readily available seismic processing packages were analyzed (Focus/Disco, Omega, Veritas, FreeUSP, Seplib, SU, MATLAB/CREWES, and ProMax). Focus/Disco was selected as the best option for ERL for the following reasons: 1) ease of use, 2) training and support availability, 3) 3-D and OBC capability, 4) cost, 5) ease of creating custom modules, and 6) hardware and software compatibility with Intel/Linux and DLT tape drives. Several Disco modules were written to extract the necessary acquisition geometry information from the headers. We will write our own analysis modules on this platform, which will provide an effective technology transfer vehicle for industry.

\section{Fracture Permeability}

We have made some initial progress on the modeling of discrete fracture networks using a simple 2-D finite difference model. These results are given in Appendix C (Sarkar et al., 2002). These results confirm our expectations that the fracture network geometry and aperture variations have a large influence on fluid flow. We will continue our modeling investigation of how to best incorporate fracture networks into simulators.

\section{CONCLUSIONS}

Good progress has been made on numerical model development and testing, both for borehole logging and surface seismic applications. Initial fluid flow modeling in fracture networks has provided a means of visualizing the fluid flow velocity field in 2-D. We will be meeting with Shell scientists to discuss field data availability in the next few months. A seismic data processing platform (Focus) is in place at ERL and our analysis modules will be developed on this platform. 


\section{REFERENCES}

Bouchon, M., and Aki, K., 1977, Discrete wave-number representation of seismic-source wave fields, Bull. Seismol. Soc. Am., 67, 259-277.

Cheng, N., 1994, 3D finite difference borehole wave propagation in isotropic and anisotropic media, $\mathrm{PhD}$ Thesis, MIT, Cambridge, MA.

Coates, R. and Schoenberg, M, 1995, Finite difference modeling of faults and fractures, Geophysics, 60, 1514-1526.

Huang, X., Sinha, B., Burns, D., Toksöz, M.N., 1999, Formation stress estimation using standard acoustic logging, $69^{\text {th }}$ Ann. Intern. Mtg. Soc. Explor. Geophys. Exp. Abs., 53-56.

Kane, J., Herrmann, F., Rodi, W., and Toksöz, M.N., 1999, Geostatistical seismic inversion using well log constraints, $6^{\text {th }}$ Ann. Intern. Mtg. Soc. Explor. Geophys. Exp. Abs., 1504-1507.

Kane, J., Rodi, W. and Toksöz, M.N., 2001, Simultaneous least squares deconvolution and kriging using conjugate gradients, 63rd Mtg. Eur. Assn. of Expl. Geophys., Session: A-24.

Shen, F., Sierra, J., Sierra, J., Burns, D.R., Burns, D.R. and Toksöz, M. N., 2002, Azimuthal offset-dependent attributes applied to fracture detection in a carbonate reservoir, Geophysics, 67, 355-364. 


\title{
A Stretched Grid Finite Difference Time-Domain Scheme Implemented With Anisotropic Perfectly Matched Layer
}

\author{
Xiaojun Huang, Yibing Zheng, Daniel R. Burns, and M. Nafi Toksöz, \\ Earth Resources Laboratory \\ Dept. of Earth, Atmospheric, and Planetary Sciences \\ Massachusetts Institute of Technology \\ Cambridge, MA 02139
}

\begin{abstract}
A stable 2.5D finite difference time-domain (FDTD) scheme is developed to study wave propagation in heterogeneous media. One example is the logging while drilling configuration where small features such as the annulus between the drill pipe and the formation, only about $1 / 10$ or even smaller than the wavelength in the steel pipe, affect the wave field significantly. The FDTD scheme proposed in this paper improves computational efficiency and accuracy from three aspects: griding, differencing, and numerical truncation. Coordinate stretching, proving to be more accurate, stable, and easy to implement, is employed to achieve variable griding. A wavelet-based differencing scheme is derived and compared with conventional FDTD schemes with spatial truncation accuracy being 2nd, 4th, 6th and 8th order. Because the wavelet-based and higher order FDTD scheme exhibits highly linear dispersion properties, it allows coarser griding and is therefore more efficient. Reflections and transmission coefficients estimated from all FDTD schemes at a sharp boundary show that the wavelet-based FDTD solution outperforms the others. Efficient numerical truncation is realized by an anisotropic perfectly matched layer.
\end{abstract}

\section{Introduction}

In order to extract information about the earth from acoustic logging observations, it is necessary to predict how wave-fields are affected by complex borehole-tool structures. Since exact solutions to the wave equations do not exist for most logging configurations, numerical simulation becomes the only viable approach to estimate such solutions.

Synthetic seismograms obtained from numerical simulation help us to predict and understand the kinematic and dynamic properties of seismic/acoustic waves propagating in the earth. They also help to determine optimal recording parameters in data acquisition and evaluate processing schemes in data processing.

Various techniques for wave modeling in complex media have been developed. Such methods include wavenumber integration, e.g. the reflectivity method (Muller, 1985), ray-tracing (Cerveny, 2001), finite elements (Chen, 1984), Fourier or pseudo-spectral methods (Kosloff and Baysal, 1982), hybrid methods (Emmerich, 1992), and finite differences (FD) (Alterman and Karal, 1968).

The FDTD method has been one of the most powerful tools to simulate wave propagation in 2-D or 3-D elastic media because it can model wave propagation accurately in arbitrarily heterogeneous media. Kelly et al. (1976) used a displacement formulation developed from the second-order elastic equations. Levander (1988) formulated a staggered-grid, finite difference scheme based on a system of first-order coupled elastic equations where the variables are stresses and velocities, rather than displacements.

Over the past 20 years, as computer capacity increased exponentially, numerical simulation using finite difference can handle more sophisticated models, from seismic wave propagation in a basin with a realistic structure, to electromagnetic wave propagating in optical fibers with fine details. However, a stable and accurate FDTD scheme with a realistic bandwidth requires a certain number of grid points per wavelength. Therefore, even with the most updated computational facilities that have dozens of central processing 
units $(\mathrm{CPU})$ and gigabytes of memory, we are still unable to simulate efficiently most models to a satisfactory level, such as wave propagation in the logging while drilling (LWD) configuration.

The FDTD scheme developed in this paper will be used primarily to investigate wave propagations in the LWD situation. A major difficulty of using the FDTD method to simulate wave propagation in the LWD situation is that small features, like the annulus between the drill pipe and the formation, affect wavefield significantly but are only about $1 / 10$ of the wavelength in the steel pipe. At least three points are required to show the effect of the annulus, and that results in a redundent griding for the rest of the model if using uniform grids. The purpose of our research, therefore, is to improve the efficiency of FDTD schemes without losing if not improving the accuracy. We examine this from three perspectives: the griding scheme, the FD operator and the numerical absorbing boundary.

\section{Non-Uniform Grid via Coordinate Stretching}

While the FDTD method developed on a uniform grid may be the simplest and most accurate scheme (Cjemg, 1994; Randall, 1991), it can be highly deficient in some respects. When modeling wave propagation along a fluid-filled borehole with a drilling pipe inside, if the grid size is not small enough to capture the thin annulus between the tool and the formation, the FDTD solution can poorly reflect the characteristics of guided modes and the coupling between them. However if the grid size is small enough to simulate the annulus, the number of grids for the whole model is far more than necessary, thus making the FDTD algorithm unnecessarily expensive to compute, including memory requirements and running time.

To avoid excessive numbers of calculations, while still obtaining a high degree of resolution in some particular regions, a non-uniform grid system may be used with a fine grid in the borehole area with small features or large changes and coarse grid over the rest of the areas that are smooth. There have been several different types of non-uniform grids applied to solve partial differential equations (PDEs). For example, it is possible to have a coarse grid that changes abruptly to a fine grid (Hayashi, 1999; Lilla, 1997), or it is possible to have a grid that changes gradually to a smaller size grid in the region of interest Anthes, 1970; Harrison, 1973).

While there are considerable benefits to be gained by having a non-uniform grid, these may be offset by other problems introduced by the change in grid size. There may be wave distortion due to a phase change at the interface of two grids or numerical reflections off the interface (Browning et al., 1973). While numerical reflection can be eliminated by taking extra averages of solutions at the grid interface, wave distortion is hard to avoid. It may also be difficult to match and implement the finite difference approximations at the interface of different grids (Hayashi, 1999; Lilla, 1997). The introduction of a change in grid spacing may also adversely affect the formal truncation error and the stability of the system (Crowder and Dalton, 1971).

Because of these difficulties, a coordinate transformation, or stretching, may be preferred to achieve an effective change in grid size. By transforming independent coordinate variables into another domain, non-uniform grid spacing is obtained in the physical domain, while uniform grid spacing is obtained in the transformed domain. Since finite difference approximation is conducted in the transformed domain where grid spacing is uniform, problems associated with non-uniform grid are eliminated naturally. Meanwhile, as grid spacing in the physical domain is non-uniform, the advantage of using non-uniform grid (saving memory and running time) is maintained. There have been several successful applications of finite difference approximation using coordinate transformation. For example, Kalnay de Rivas achieved a large improvement in accuracy when simulating the circulation of the atmosphere on Venus (Kalnay de Rivas, 1972). However, little effort has been put into solving elastic wave equations with finite difference approximation using coordinate transformation.

In this section, we will construct a stretching grid finite difference approximation for the elastic wave equations.

Coordinate stretching is a coordinate transformation that maps the physical domain, $\vec{x}=\left[x_{1}, x_{2}, x_{3}\right]^{T}$, to the transformed or stretched domain, $\overrightarrow{\tilde{x}}=\left[\tilde{x}_{1}, \tilde{x}_{2}, \tilde{x}_{3}\right]^{T}$, by a grid mapping function $\epsilon^{i}\left(\tilde{x}_{i}\right)$, where $i=1,2,3$, representing three orthogonal coordinate directions. $\epsilon^{i}\left(\tilde{x}_{i}\right)$ should be continuous with respect to $\tilde{x}_{i}$, at least to the order of the PDE that is to be solved. $\epsilon^{i}\left(\tilde{x}_{i}\right)$ is constructed in the manner that the grid spacing in the 
stretched coordinate, $d \tilde{x}_{i}$, is uniform whereas grid spacing in the physical coordinate, $d x_{i}$ is non-uniform.

$$
d x_{i}=\epsilon^{i}\left(\tilde{x}_{i}\right) d \tilde{x}_{i}
$$

In this paper, $\epsilon^{i}\left(\tilde{x}_{i}\right)$ is built as follows:

$$
\epsilon^{i}\left(\tilde{x}_{i}\right)=\left\{\begin{array}{cc}
1 & \tilde{x}_{i} \leqslant \tilde{x}_{i}^{0} \\
1+(\alpha-1) \sin \frac{\pi}{2 L}\left(\tilde{x}_{i}-\tilde{x}_{i}^{0}\right) & \tilde{x}_{i}^{0} \leqslant \tilde{x}_{i} \leqslant \tilde{x}_{i}^{0}+L \\
\alpha & \tilde{x}_{i} \geqslant \tilde{x}_{i}^{0}+L
\end{array}\right.
$$

where $\alpha$ is the stretching parameter that controls the effective velocity in the transformed domain. It is also the ratio between the coarse and the fine grid in the physical domain. Note that unlike the abrupt grid changing scheme Hayashi, 1999; Lillas, 1997), $\alpha$ does not have to be an integer. $L$ is the transition length. Figure 1 shows the shape of the stretching function, $\epsilon(\tilde{x})$. When $\epsilon$ is $1, d x=d \tilde{x}$, the grid spacing

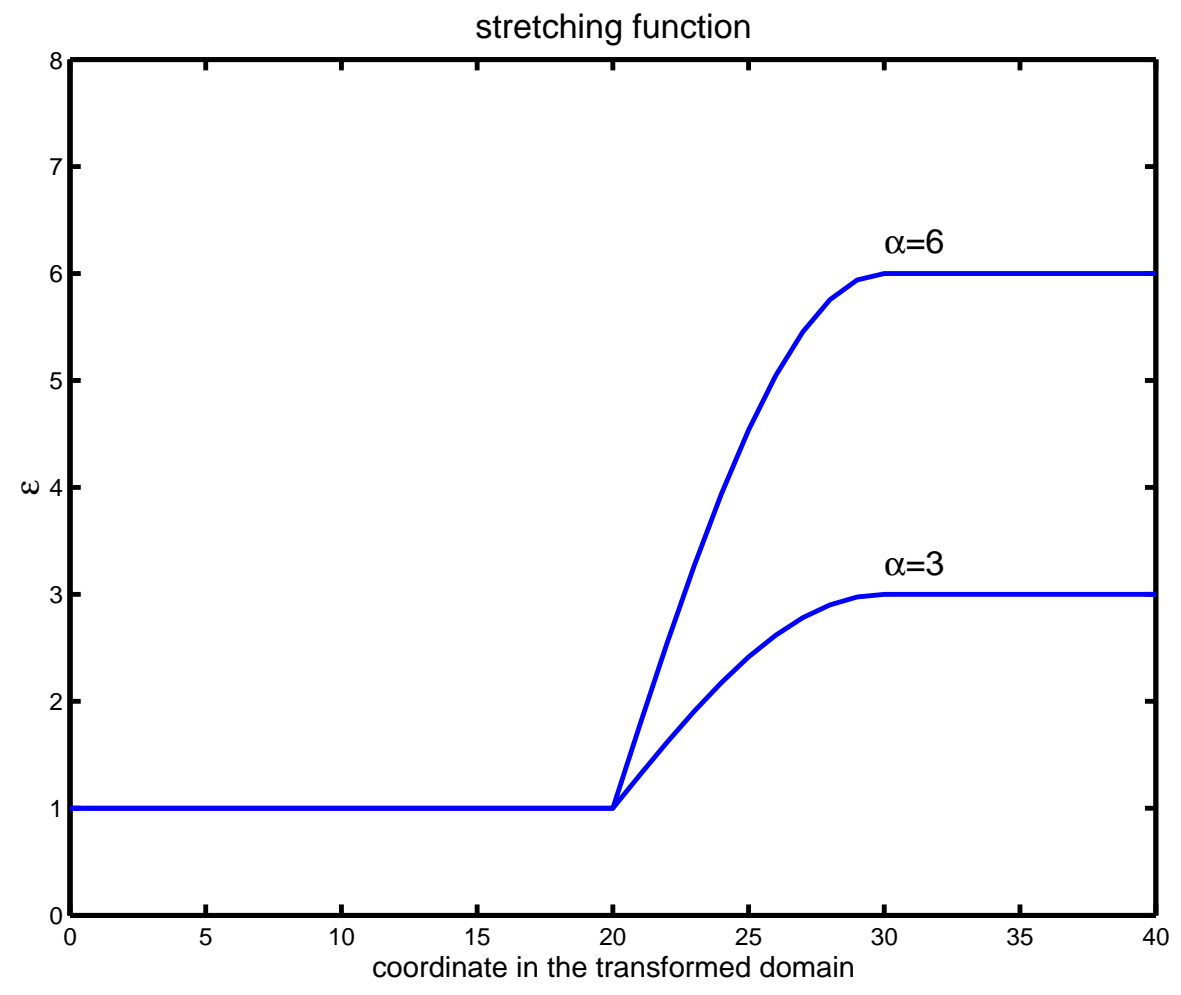

Figure 1: The stretching function $\epsilon(\tilde{x})$ for different stretching parameters.

in the physical domain is the same as in the transformed domain. When $\epsilon$ takes some other value, the grid spacing in the physical domain is $\epsilon$ times larger than it in the transformed domain; therefore, grid spacing in the physical domain is non-uniform, whereas it is uniform in the transformed domain. The wave equations are then solved in the transformed domain using finite difference approximation with uniform griding, thus avoiding the problems that occur with non-uniform grids.

Integrate equation 1 , the explicit mapping relation between the physical domain $\vec{x}=\left[x_{1}, x_{2}, x_{3}\right]^{T}$ and the stretching domain $\overrightarrow{\tilde{x}}=\left[\tilde{x}_{1}, \tilde{x}_{2}, \tilde{x}_{3}\right]^{T}$ is established as

$$
x_{i}=\left\{\begin{array}{cc}
\tilde{x}_{i} & \tilde{x}_{i} \leqslant \tilde{x}_{i}^{0} \\
\tilde{x}_{i}+(\alpha-1) \frac{2 L}{\pi}\left\{1-\cos \frac{\pi}{2 L}\left(\tilde{x}_{i}-\tilde{x}_{i}^{0}\right)\right. & \left.\tilde{x}_{i}^{0} \leqslant \tilde{x}_{i} \leqslant \tilde{x}_{i}^{0}+L\right\} \\
(1-\alpha)\left(\tilde{x}_{i}^{0}+L-\frac{2 L}{\pi}\right)+\alpha \tilde{x}_{i} & \tilde{x}_{i} \geqslant \tilde{x}_{i}^{0}+L
\end{array}\right.
$$


Figures 2 and 3 illustrate grid spacing in both domains. The figures show that the model space becomes smaller after coordinate transformation, requiring less memory and running time. Applying the chain rule, the elastic wave equations after the coordinate transformation can be written as,

$$
\begin{array}{r}
\rho v_{j, t}=\frac{1}{\epsilon^{\alpha}\left(\tilde{x}_{\alpha}\right)} \tau_{\alpha j, \alpha} \\
\tau_{\alpha j, t}=c_{\alpha j \gamma \beta} \frac{1}{\epsilon^{\beta}\left(\tilde{x}_{\beta}\right)} v_{\gamma, \beta}
\end{array}
$$

where $\alpha, j, \gamma$ and $\beta$ take the values of 1,2 and 3. Equations 4 and 5 show that the effective wave velocity is slower in the transformed domain, which is compensated by a smaller model size; hence the total travel time is the same as in the physical domain.

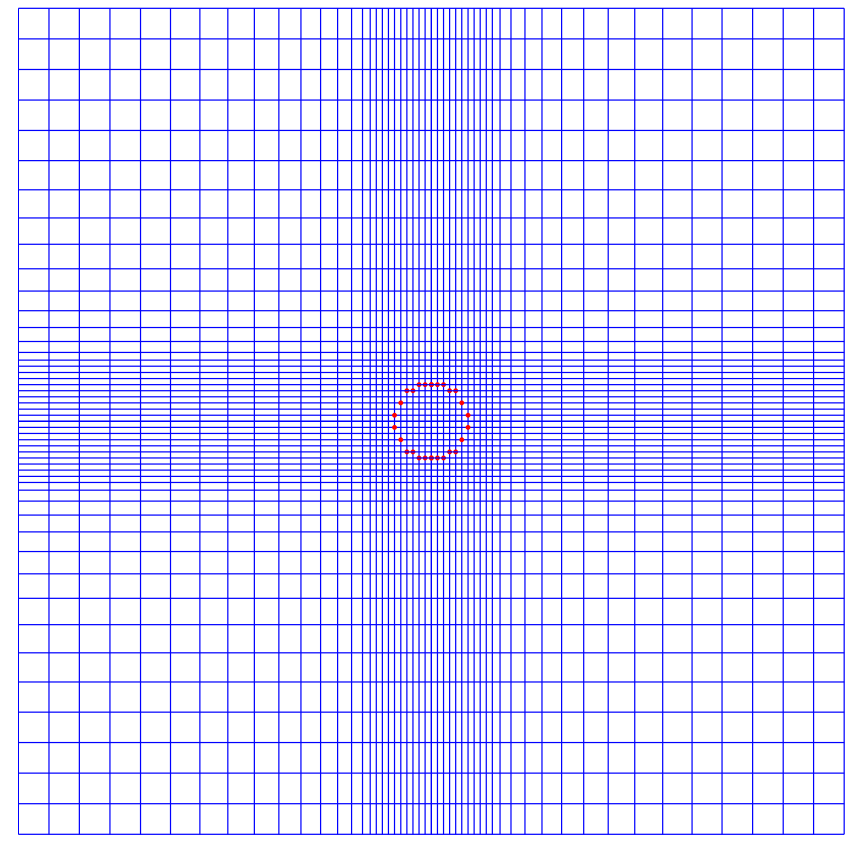

Figure 2: Non-uniform grid spacing in the physical domain. The stretching ratio is 5 .

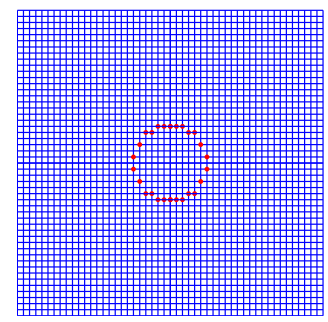

Figure 3: Uniform grid spacing in the transformed domain. Comparing to Figure 2, the model space becomes much smaller thus saving memory and running time. 


\section{Anisotropic Perfectly Matched Layer}

In most cases, we need to simulate wave propagations in a boundless medium, hence the medium is truncated into a finite size. This requires numerically implementing an absorbing boundary layer surrounding the computational domain to reduce reflections off the numerical boundary. An inefficient absorbing boundary layer results in a less accurate and less efficient algorithm.

Berenger (1994) first proposed the perfectly matched layer (PML) concept for electrodynamics using the FDTD method. Different from most traditional or differential equation-based absorbing boundaries, the PML satisfies all continuity conditions at the interface between the computational domain and the PML. Therefore, it has proven to be the most efficient mechanism to absorb wave energies outside the computational domain (Taflove, 1998). Liu formulated the PML for elastic waves using the field-splitting method which requires 27 independent unknowns for a general 3-D problem (9 velocity components and 18 stress components), three times the original 9 variables in ordinary elastic wave equations (Liu, 1999). Zheng and Huang formulated an anisotropic PML without using field splitting that only requires 12 independent unknowns for a general 3-D problem, and showed superior results using the finite element method (Zheng and Huang, 2001). In this paper, further study shows that 21 independent unknowns are required for a stable FDTD PML.

Suppose that any real medium where a plane wave is traveling with a wavenumber $\vec{k}$, its perfectly matched layer is an artificial medium where the same plane wave travels with a complex wavenumber $\overrightarrow{k^{p}}$, the real part of that wavenumber $\operatorname{Re}\left(\overrightarrow{k^{p}}\right)$ equals to $\vec{k}$. If the medium and its perfectly matched layer are placed next to each other, there will be no reflections or other transmissions generated (Taflove, 1998). Since the wavenumber is complex, the plane wave is attenuated inside the PML. Because any wave with an arbitrary wavefront can be considered as a linear combination of a set of plane waves traveling in different directions, this wave is also attenuated in the PML and can travel across the real medium and its perfectly matched layer without generating any reflections or other transmissions at the interface. So, if such PMLs can be found for any real media, in numerical computation, we can always put them around the computational domain to simulate wave propagation in a boundless medium.

Zheng and Huang (2001) proved that the PML of any real medium exists and provided a systematic way to find it for general anisotropic media in Cartesian and curvilinear coordinates. It is worth mentioning that PML is usually anisotropic. In this section, PML is derived for a stretched coordinate and applied to the FDTD algorithm.

\subsection{Formulation}

Inside the PML, according to the formulation in Zheng and Huang (2001), equation 4 becomes

$$
\rho e_{1} e_{2} e_{3} v_{j, t}=\frac{1}{\epsilon^{\alpha}\left(\tilde{x}_{\alpha}\right)} \tau_{\alpha j, \alpha}^{\mathrm{PML}}
$$

Let tensor $\overline{\bar{\tau}}^{\mathrm{PML}}=\tau_{\alpha j}^{\mathrm{PML}}$, denoting stress components in the PML, it is related to the stress tensor $\overline{\bar{\tau}}=\tau_{\alpha j}$ in the computational domain next to the PML by

$$
\overline{\bar{\tau}}^{\mathrm{PML}}=e_{1} e_{2} e_{3}\left[\begin{array}{lll}
\frac{1}{e_{1}} & & \\
& \frac{1}{e_{2}} & \\
& & \frac{1}{e_{3}}
\end{array}\right] \overline{\bar{\tau}}
$$

Complex numbers $e_{1}, e_{2}$ and $e_{3}$ are chosen such that normal and tangential stress components are continuous across the boundary between the computational domain and the PML. Writing every component explicitly in the following, equation 7 shows how stress continuity is achieved.

$$
\begin{array}{rll}
\tau_{x x}^{\mathrm{PML}}=e_{2} e_{3} \tau_{x x}, & \tau_{x y}^{\mathrm{PML}}=e_{2} e_{3} \tau_{x y}, & \tau_{x z}^{\mathrm{PML}}=e_{2} e_{3} \tau_{x z}, \\
\tau_{y x}^{\mathrm{PML}}=e_{1} e_{3} \tau_{y x}, & \tau_{y y}^{\mathrm{PML}}=e_{1} e_{3} \tau_{y y}, & \tau_{y z}^{\mathrm{PML}}=e_{1} e_{3} \tau_{y z}, \\
\tau_{z x}^{\mathrm{PML}}=e_{1} e_{2} \tau_{z x}, & \tau_{z y}^{\mathrm{PML}}=e_{1} e_{2} \tau_{z y}, & \tau_{z z}^{\mathrm{PML}}=e_{1} e_{2} \tau_{z z} .
\end{array}
$$


Let $e_{1}=1-i \zeta_{1}, e_{2}=1-i \zeta_{2}$ and $e_{3}=1-i \zeta_{3}$. Suppose elastic waves traveling at all angles hit the numerical boundary with the normal direction coincidence with $x$ coordinate, if $\zeta_{2}$ and $\zeta_{3}$ are chosen to be 0 and $\zeta_{1}$ increases gradually from 0 to some finite number away from the numerical boundary, stress components including normal and tangential components acting on the numerical boundary are continuous across the boundary, i.e., $\tau_{x x}=\tau_{x x}^{\mathrm{PML}}, \tau_{x y}=\tau_{x y}^{\mathrm{PML}}$ and $\tau_{x z}=\tau_{x z}^{\mathrm{PML}}$. Note that the stress tensor in the PML, $\tau_{i j}^{\mathrm{PML}}$ is not symmetric. Details of selecting $e_{1}, e_{2}$ and $e_{3}$ will be illustrated in section 5 .

In vector and tensor format, equation 5 can be written as two separate equations

$$
\begin{array}{r}
{\left[\begin{array}{ccc}
\frac{1}{e_{2} e_{3}} & & \\
& \frac{1}{e_{1} e_{3}} & \\
& \frac{1}{e_{1} e_{2}}
\end{array}\right] \frac{\partial \overline{\bar{\tau}}^{\mathrm{PML}}}{\partial t}=\mathbf{C}: \overline{\bar{E}}^{\mathrm{PML}}} \\
\\
{\left[\begin{array}{ccc}
e_{1} & & \\
& e_{2} & \\
& & e_{3}
\end{array}\right] \overline{\bar{E}}^{\mathrm{PML}}=\nabla \vec{v}}
\end{array}
$$

where $\overline{\bar{E}}^{\mathrm{PML}}$ has 9 components, representing the strain rate in the PML. The benefit of dividing equation 5 into equations 11 and 12 , and introducing the strain rate, $\overline{\bar{E}}^{\mathrm{PML}}$, is to have a stable and simple FDTD algorithm, far beating the cost of requiring slightly more computation and memory inside PML. Let $e_{1} e_{2} e_{3}=$ $A-i B / \omega$, where $A, B \in \mathbb{R}, \omega$ is an arbitrary angular frequency, then Fourier transform equation 6 , apply the relationship of $i \omega=1 / \partial t$, and then inverse Fourier transform, in vector form, equation 6 becomes

$$
\rho A \partial_{t} \vec{v}+\rho B \vec{v}=\tilde{\nabla}_{v} \vec{\tau}^{\mathrm{PML}}
$$

where $\vec{v}=\left[v_{x}, v_{y}, v_{z}\right]^{T}$ and $\vec{\tau}^{\mathrm{PML}}=\left[\tau_{x x}^{\mathrm{PML}}, \tau_{y y}^{\mathrm{PML}}, \tau_{z z}^{\mathrm{PML}}, \tau_{x y}^{\mathrm{PML}}, \tau_{y x}^{\mathrm{PML}}, \tau_{x z}^{\mathrm{PML}}, \tau_{z x}^{\mathrm{PML}}, \tau_{y z}^{\mathrm{PML}}, \tau_{z y}^{\mathrm{PML}}\right]^{T}$.

$$
\tilde{\nabla}_{v}=\left[\begin{array}{ccccccccc}
\frac{1}{\epsilon^{1}(\tilde{x})} \partial_{\tilde{x}} & 0 & 0 & 0 & \frac{1}{\epsilon^{2}(\tilde{y})} \partial_{\tilde{y}} & 0 & \frac{1}{\epsilon^{3}(\tilde{z})} \partial_{\tilde{z}} & 0 & 0 \\
0 & \frac{1}{\epsilon^{2}(\tilde{y})} \partial_{\tilde{y}} & 0 & \frac{1}{\epsilon^{1}(\tilde{x})} \partial_{\tilde{x}} & 0 & 0 & 0 & 0 & \frac{1}{\epsilon^{3}(\tilde{z})} \partial_{\tilde{z}} \\
0 & 0 & \frac{1}{\epsilon^{3}(\tilde{z})} \partial_{\tilde{z}} & 0 & 0 & \frac{1}{\epsilon^{1}(\tilde{x})} \partial_{\tilde{x}} & 0 & \frac{1}{\epsilon^{2}(\tilde{y})} \partial_{\tilde{y}} & 0
\end{array}\right]
$$

Following a similar approach, as taken for equation 6, equation 11 becomes

$$
\Pi \vec{\tau}^{\mathrm{PML}}=\mathbf{C} \vec{E}^{\mathrm{PML}}
$$

where $\vec{E}^{\mathrm{PML}}=\left[E_{x x}^{\mathrm{PML}}, E_{y y}^{\mathrm{PML}}, E_{z z}^{\mathrm{PML}}, E_{x y}^{\mathrm{PML}}, E_{y x}^{\mathrm{PML}}, E_{x z}^{\mathrm{PML}}, E_{z x}^{\mathrm{PML}}, E_{y z}^{\mathrm{PML}}, E_{z y}^{\mathrm{PML}}\right]^{T}$; Components of matrix $\Pi$ and $\mathbf{C}$ are listed below.

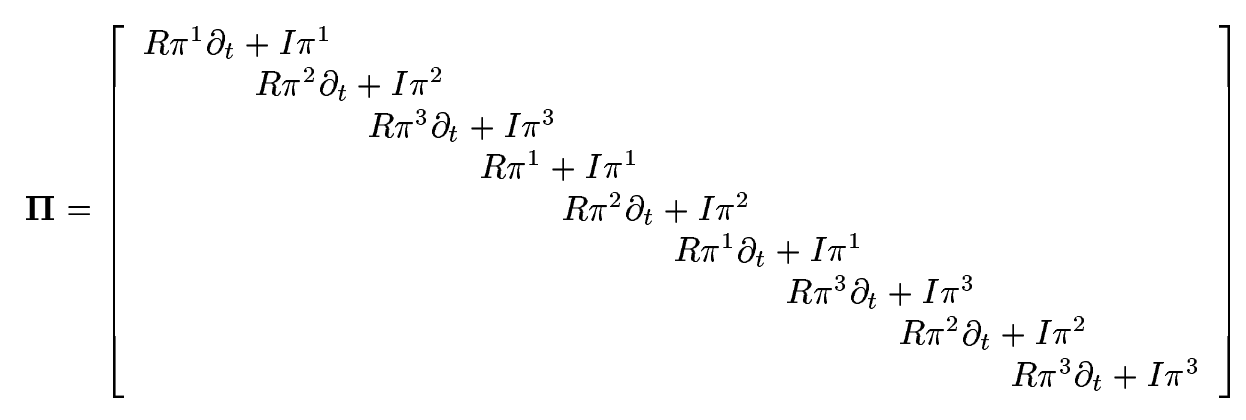

where

$$
\begin{aligned}
R \pi^{1} & =\frac{1-\zeta_{2} \zeta_{3}}{\left(1-\zeta_{2} \zeta_{3}\right)^{2}+\left(\zeta_{2}+\zeta_{3}\right)^{2}}, & I \pi^{1} & =-\frac{\left(\zeta_{2}+\zeta_{3}\right) \omega}{\left(1-\zeta_{2} \zeta_{3}\right)^{2}+\left(\zeta_{2}+\zeta_{3}\right)^{2}} \\
R \pi^{2} & =\frac{1-\zeta_{1} \zeta_{3}}{\left(1-\zeta_{1} \zeta_{3}\right)^{2}+\left(\zeta_{1}+\zeta_{3}\right)^{2}}, & I \pi^{2} & =-\frac{\left(\zeta_{1}+\zeta_{3}\right) \omega}{\left(1-\zeta_{1} \zeta_{3}\right)^{2}+\left(\zeta_{1}+\zeta_{3}\right)^{2}} \\
R \pi^{3} & =\frac{1-\zeta_{1} \zeta_{2}}{\left(1-\zeta_{1} \zeta_{2}\right)^{2}+\left(\zeta_{1}+\zeta_{2}\right)^{2}}, & I \pi^{3} & =-\frac{\left(\zeta_{1}+\zeta_{2}\right) \omega}{\left(1-\zeta_{1} \zeta_{2}\right)^{2}+\left(\zeta_{1}+\zeta_{2}\right)^{2}}
\end{aligned}
$$




$$
\mathbf{C}=\left[\begin{array}{lllllllll}
c_{11} & c_{12} & c_{13} & & & & & & \\
c_{12} & c_{22} & c_{23} & & & & & & \\
c_{13} & c_{23} & c_{33} & & & & & & \\
& & & c_{66} & c_{66} & & & & \\
& & & c_{66} & c_{66} & & & & \\
& & & & & c_{55} & c_{55} & & \\
& & & & & c_{55} & c_{55} & & \\
& & & & & & & c_{44} & c_{44} \\
& & & & & & & c_{44} & c_{44}
\end{array}\right]
$$

Similarly, equation 12 becomes

$$
\Xi \vec{E}^{\mathrm{PML}}=\tilde{\nabla}_{E} \vec{v}
$$

where

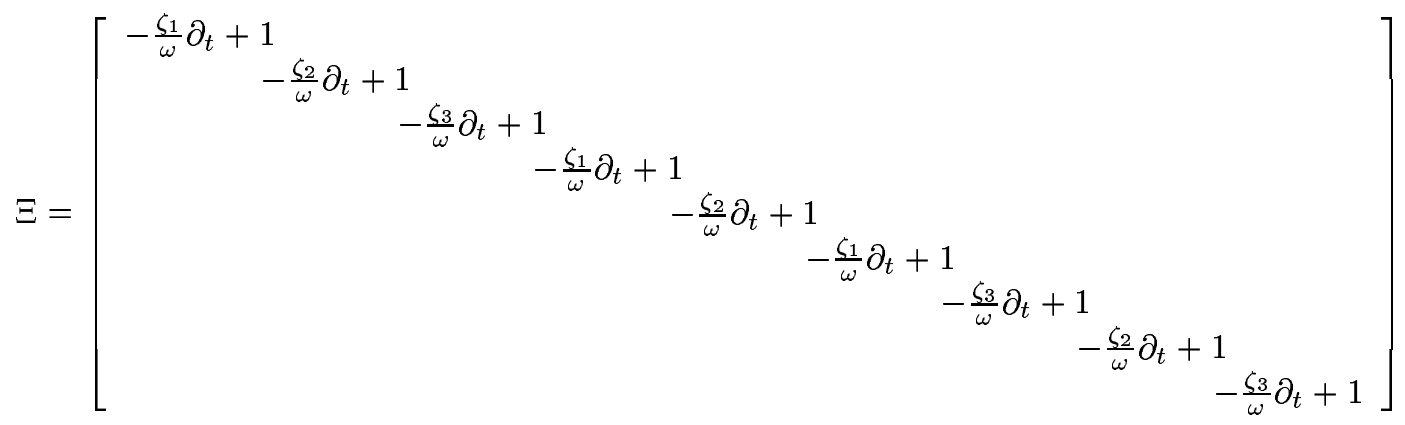

and

$$
\tilde{\nabla}_{E}=\left[\begin{array}{ccc}
\frac{1}{\epsilon^{1}(\tilde{x})} \partial_{\tilde{x}} & & \\
& \frac{1}{\epsilon^{2}(\tilde{y})} \partial_{\tilde{y}} & \frac{1}{\epsilon^{3}(\tilde{z})} \partial_{\tilde{z}} \\
\frac{1}{\epsilon^{2}(\tilde{y})} \partial_{\tilde{y}} & \frac{1}{\epsilon^{1}(\tilde{x})} \partial_{\tilde{x}} & \\
\frac{1}{\epsilon^{3}(\tilde{z})} \partial_{\tilde{z}} & & \frac{1}{\epsilon^{1}(\tilde{x})} \partial_{\tilde{x}} \\
& \frac{1}{\epsilon^{3}(\tilde{z})} \partial_{\tilde{z}} & \frac{1}{\epsilon^{2}(\tilde{y})} \partial_{\tilde{y}}
\end{array}\right]
$$

Until now, the wave equations in the PML have been derived in the transformed coordinate domain (equations 13, 15 and 21). There are two remarkable properties about those equations. 1) Though orthorhombic anisotropy is assumed throughout the paper, or explicitly specified otherwise, those equations can deal with general anisotropic media, therefore the PML designed using those equations can attenuate numerical energies even when the medium in the computational domain is anisotropic. 2) When the parameters $\zeta_{1}, \zeta_{2}$ and $\zeta_{3}$, which are associated with absorbing coefficients, are set to be zeros, those equations yield normal elastic wave equations in the transformed coordinate. Since medium properties are kept the same in the PML as in neighboring computational domain, no reflections will happen at the numerical boundary between the computational domain and the PML. So in the PML, $\zeta_{1}, \zeta_{2}$ and $\zeta_{3}$ are set to be zeros at the neighborhood of the numerical boundary and gradually increased toward the outer bound of the PML.

\section{Staggered-Grid Finite Difference Time-Domain Scheme: A Wavelet Based Formulation and Higher Order Method}

By approximating derivatives with differences, the finite difference scheme converts PDEs with linear algebraic equations. The discrepancy between the finite difference solution and the PDE solution consists of 
two contributions: the discretization error that causes grid dispersion and anisotropy, and the truncation error due to cutting the infinite long operator into a finite length. Techniques for obtaining a more accurate numerical solution using FDTD has been focused on reducing those two effects, such as using higher-order difference approximations (Dablain, 1986) and staggered grids (Virieux, 1986).

In recent years, there have been several successful applications of wavelet theory to time domain schemes for electrodynamics, based on the method of moments (Harrington, 1993). This method expands wave field components (particle velocities and stresses) to a complete set of orthonormal basis functions, then discretizes the expansion coefficients by taking inner product with a test function which is orthogonal to the basis function. It was shown that the conventional staggered grid FDTD scheme can be derived using the method of moments with pulse functions being the orthonormal basis and test functions Krumpholz and Russer, 1993, 1994). A multiresolution time-domain (MRTD) scheme based on wavelet theory was developed for electrodynamics and exhibits highly linear numerical dispersion characteristics, therefore allowing coarser grid spacing (Krumpholz and Katehi, 1996). In their work, Krumpholz and Katehi employed the BattleLemarie scaling and wavelet functions as basis functions. Because those basis functions do not satisfy the interpolation property, the expansion coefficients do not represent direct physical wave field values. Hence it becomes necessary to reconstruct the physical field by taking a weighted sum of neighboring coefficients, resulting in a complicated algorithm and a large computational overhead. In 2001, Fujii and Hoefer avoided the step of reconstruction by using the Deslauriers-Dubuc interpolating functions Dubuc, 1986; Deslauriers and Dubuc, 1989) as the basis functions (Fujii and Hoefer, 2001).

Thus far the wavelet formulation of the FDTD method has not been studied solving elastic wave equations. In this section, we develop a time-domain scheme using a wavelet-based differencing approach DeslauriersDubuc interpolating functions to solve the elastic wave equations. The spatial derivative is approximated by Deslauriers-Dubuc interpolating functions and the temporal derivative is approximated by the Harr scaling function (Strang, 1996). The Harr scaling function is a compactly supported step function shown in Figure 4. A comparison of numerical dispersion and accuracy between the wavelet-based scheme and conventional FDTD of order 2, 4, 6 and 8 shows that the wavelet based scheme exhibits similar linear dispersion characteristics as higher order method (6th or 8th order), resulting in a reduction in grid points per wavelength necessary to maintain the same, if not better accuracy than the 4th order scheme. FDTD results from the 1-D wave equation show that wavelet-base formulation produce more accurate reflection and transmission at sharp boundaries.

\subsection{Interpolating Basis Functions}

The Deslauriers-Dubuc interpolating function $\phi$ of order $2 p-1$ is the autocorrelation function of the Daubechies compactly supported orthogonal scaling function $\phi_{0}$ of $p$ vanishing wavelet moments (Mallat, 1997),

$$
\phi(x)=\int_{-\infty}^{\infty} \phi_{0}(u) \phi_{0}(u-x) d u .
$$

$\phi$ is compactly supported, has a minimum support of $[-2 p+1,2 p-1]$ and is able to reproduce polynomials of order $2 p-1$. In addition, being an autocorrelation function, $\phi$ is symmetric. Figures 5 and 6 illustrate Daubechies scaling function and Deslauriers-Dubuc interpolating function. Note that $\phi(x)$ is orthogonal to its integer shift; therefore functions being discretized and expanded to a family of $\phi(x)$ do not require extra computation to be reconstructed. A remarkable property of $\phi$ is that it can be written as a sum of scaled and translated copies of itself. Such a property is called two-scale relation or dilation relation which is denoted as

$$
\phi(x)=\sum_{k=-\infty}^{+\infty} h_{k}^{*} \phi(2 t-k) .
$$

where coefficients $h_{k}^{*}$ is obtained by taking the autocorrelation of Daubechies wavelet filter $h_{k}$ (Daubechies, 1988). Values of $h_{k}^{*}$ for $p=2,4$, and 6 are listed in table 4.1. As an initial work, only the scaling functions are chosen as the basis function, therefore $h_{k}$ is the low-pass decomposition filter of Daubechies wavelet filter family. Results of electrodynamics show that by using only the scaling function itself still lead to savings in the number of grids due to the highly linear dispersion property of the resulting scheme. Incorporating 


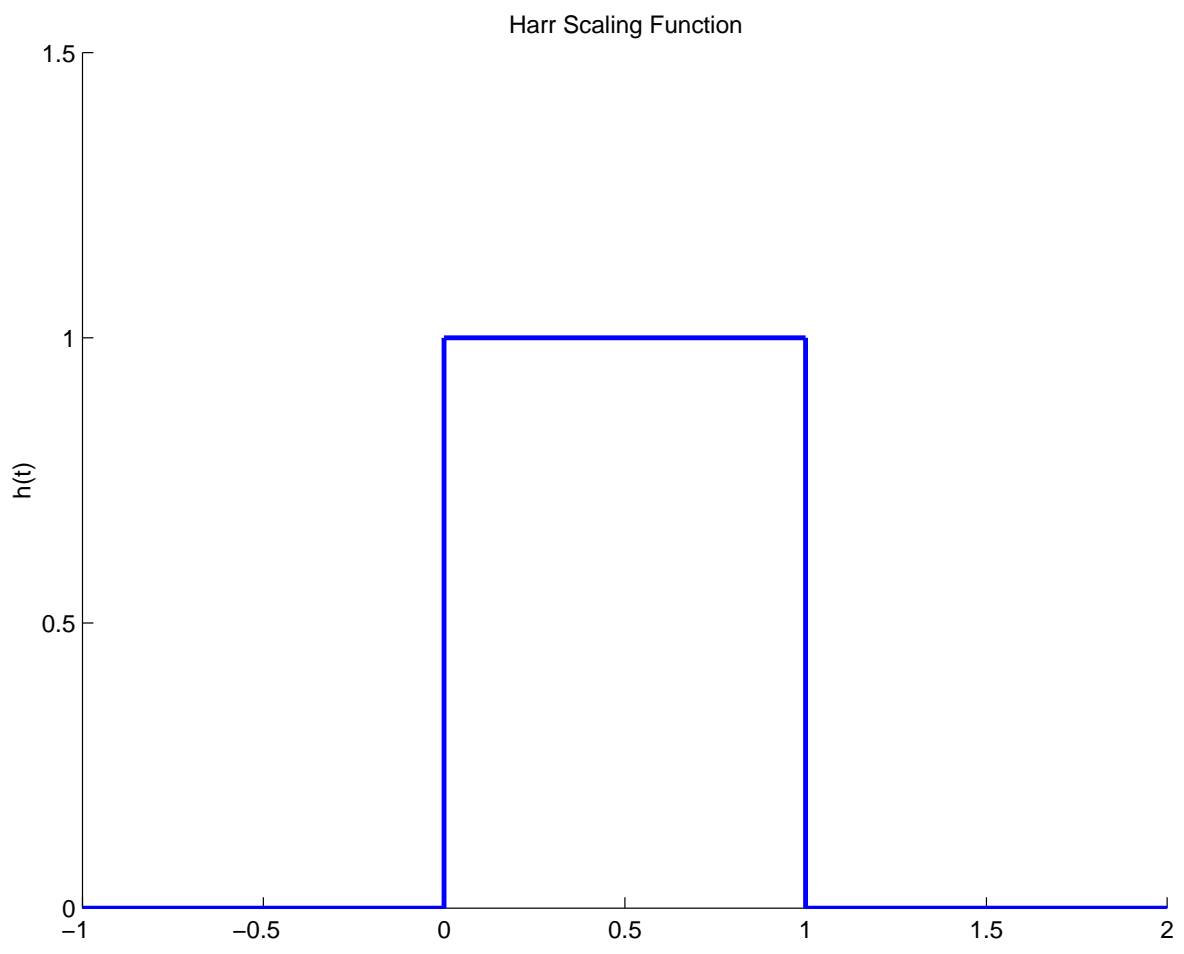

Figure 4: Harr scaling function $h(t)$.

wavelet functions will possibly yield higher resolution schemes. Dirac delta function $\delta(x)$ is chosen to be the dual function of $\phi(x)$, the Deslauriers-Dubuc interpolating function. Denoting the discretization of a function $f(x)$ as

$$
f_{j}(x)=f\left(\frac{x}{\Delta x}-j\right)
$$

where $f(x)$ represents $\phi(x)$ and its dual function, $\delta(x) . \Delta x$ is the grid spacing. Since $f(x)$ is orthogonal to integer shift, $f_{j}(x)$ is orthogonal to shifts equal to integer times of $\Delta x$. The inner product of $\phi_{i}(x)$ and $\delta_{j}(x)$ satisfies the following biorthogonal relation

$$
<\phi_{i}, \delta_{j}>=\delta_{i j}
$$

where $\delta_{i j}$ is the Kronecker delta function. Similar to Deslauriers-Dubuc interpolating function, Harr scaling function is also orthogonal to its integer shift. In order to construct staggered-grid FDTD scheme, Harr scaling function is left shifted by $\frac{1}{2}$ then the corresponding discretization is defined as

$$
h_{n}(t)=h\left(\frac{t}{\Delta t}-n+\frac{1}{2}\right)
$$




\begin{tabular}{|c|c|c|c|}
\hline $\mathrm{k}$ & $\mathrm{p}=2$ & $\mathrm{p}=4$ & $\mathrm{p}=6$ \\
\hline 0 & 1.00000000 & 1.00000000 & 1.00000000 \\
1 & 0.56250000 & 0.59814453 & 0.61016818 \\
2 & 0.00000000 & 0.00000000 & 0.00000000 \\
3 & -0.06250000 & -0.11962891 & -0.14539719 \\
4 & & 0.00000000 & 0.00000000 \\
5 & & 0.02392578 & 0.04361916 \\
6 & & 0.00000000 & 0.00000000 \\
7 & & -0.00244141 & -0.01038551 \\
8 & & & 0.00000000 \\
9 & & & 0.00161552 \\
10 & & & 0.00000000 \\
11 & & & -0.00012016 \\
\hline
\end{tabular}

Table 1: Filter coefficients $h_{k}^{*}$ in equation 25 .

\begin{tabular}{|c|c|c|}
\hline $\mathrm{l}$ & $\mathrm{p}=2$ & $\mathrm{p}=4$ \\
\hline 0 & 1.2291666667 & 1.3110340773 \\
1 & -0.0937500000 & -0.1560100710 \\
2 & 0.0104166667 & 0.0419957460 \\
3 & & -0.0086543236 \\
4 & & 0.0008308695 \\
5 & & 0.0000108999 \\
6 & & -0.0000000041 \\
\hline
\end{tabular}

Table 2: $\mathrm{a}(\mathrm{l})$ in equation $32 . a(-l)=-a(l-1)$.

where $\Delta t$ is time step. Similar to $f_{j}(x), h_{n}(t)$ is orthogonal to shifts equal to integer times of $\Delta t$. The orthogonality conditions are listed below explicitly.

$$
\begin{array}{lc}
\int_{x=-\infty}^{+\infty} & \phi_{i}(x) \delta_{i^{\prime}}(x) d x=\delta_{i, i^{\prime}} \Delta x \\
\int_{t=-\infty}^{+\infty} & h_{n}(t) h_{n^{\prime}}(t) d t=\delta_{n, n^{\prime}} \Delta t \\
\int_{t=-\infty}^{+\infty} & \partial_{t} h_{n+1 / 2}(t) h_{n^{\prime}}(t) d t=\delta_{n, n^{\prime}}-\delta_{n+1, n^{\prime}} \\
\int_{x=-\infty}^{+\infty} & \partial_{x} \phi_{i+1 / 2}(x) \delta_{i^{\prime}}(x) d x=\left.\frac{d \phi) x)}{d x}\right|_{x=i^{\prime}-i-\frac{1}{2}} \equiv a(l), \quad l=i-i^{\prime}
\end{array}
$$

$a(l)$ in equation 32 is evaluated numerically and are listed in Table 4.1 for $p=2$ and 4.

\subsection{Discretization of Elastic Wave Equations}

A staggered-grid scheme is employed to discretize the elastic wave equation as it is more stable and accurate. Because spatial derivatives with respect to $x, y$ and $z$ in the wave equations are decoupled, illustrating the discretization using Deslauriers-Dubuc interpolating function for a 1-D wave equation will not lose generality. The formulation for the full 3-D wave equation in the stretched coordinated is listed at the end of this subsection. 

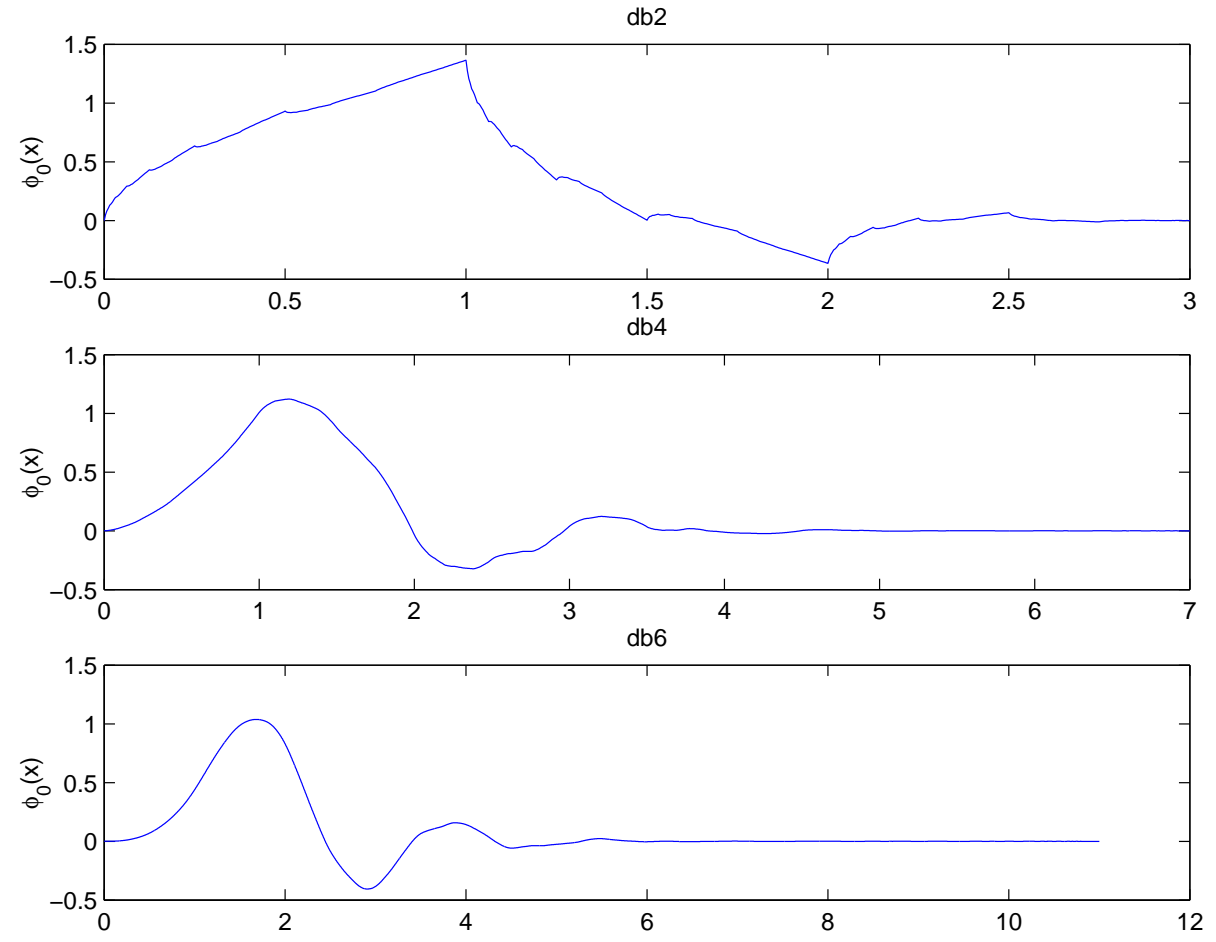

Figure 5: Daubechies compactly supported scaling function $\phi_{0}$ of 2, 4, and 6 vanishing moments.

\subsubsection{Discretization of 1-D Wave Equation}

The set of 1-D wave equation that needs discretization is

$$
\begin{array}{r}
\rho(x) \partial_{t} v_{x}(x, t)=\partial_{x} \tau_{x x}(x, t) \\
\partial_{t} \tau_{x x}(x, t)=c_{11} \partial_{x} v_{x}(x, t)
\end{array}
$$

where the normal stress $\tau_{x x}(x, t)$ and particle velocity $v_{x}(x, t)$ can be represented by linear combinations of discretized Deslauriers-Dubuc interpolating function $\phi_{j}(x)$ in space and Harr scaling function $h_{n}(t)$ in time

$$
\begin{gathered}
\tau_{x x}(x, t)=\sum_{i, n=-\infty}^{+\infty} \tau_{i+1 / 2 ; n}^{x x} \phi_{i+1 / 2}(x) h_{n}(t) \\
v(x, t)=\sum_{i, n=-\infty}^{+\infty} v_{i ; n+1 / 2}^{x} \phi_{i}(x) h_{n+1 / 2}(t)
\end{gathered}
$$

After substituting equations 35 and 36 to 33 , taking inner product of both sides with testing functions $\delta_{i^{\prime}}(x)$ and $h_{n^{\prime}}(t)$ may yields

$$
\begin{aligned}
& \int_{x=-\infty}^{+\infty} \int_{t=-\infty}^{+\infty} \partial_{t} \sum_{i=-\infty}^{+\infty} \sum_{n=-\infty}^{+\infty} \rho_{i} v_{i ; n+1 / 2}^{x} \phi_{i}(x) h_{n+1 / 2}(t) \delta_{i^{\prime}}(x) h_{n^{\prime}}(t) d x d t \\
& =\int_{x=-\infty}^{+\infty} \int_{t=-\infty}^{+\infty} \partial_{x} \sum_{i=-\infty}^{+\infty} \sum_{n=-\infty}^{+\infty} \tau_{i+1 / 2 ; n}^{x x} \phi_{i+1 / 2}(x) \delta_{i^{\prime}}(x) h_{n}(t) h_{n^{\prime}}(t) d x d t
\end{aligned}
$$

Applying orthogonality conditions to the above equation leads to the discretization of equation 33

$$
v_{i^{\prime} ; n^{\prime}+1 / 2}^{x}=v_{i^{\prime} ; n^{\prime}-1 / 2}^{x}+\frac{\Delta t}{\rho_{i^{\prime}} \Delta x} \sum_{l=0}^{L s-1} a(l)\left(\tau_{i^{\prime}+\frac{2 l-1}{2} ; n^{\prime}}^{x x}-\tau_{i^{\prime}-\frac{2 l-1}{2} ; n^{\prime}}^{x x}\right)
$$




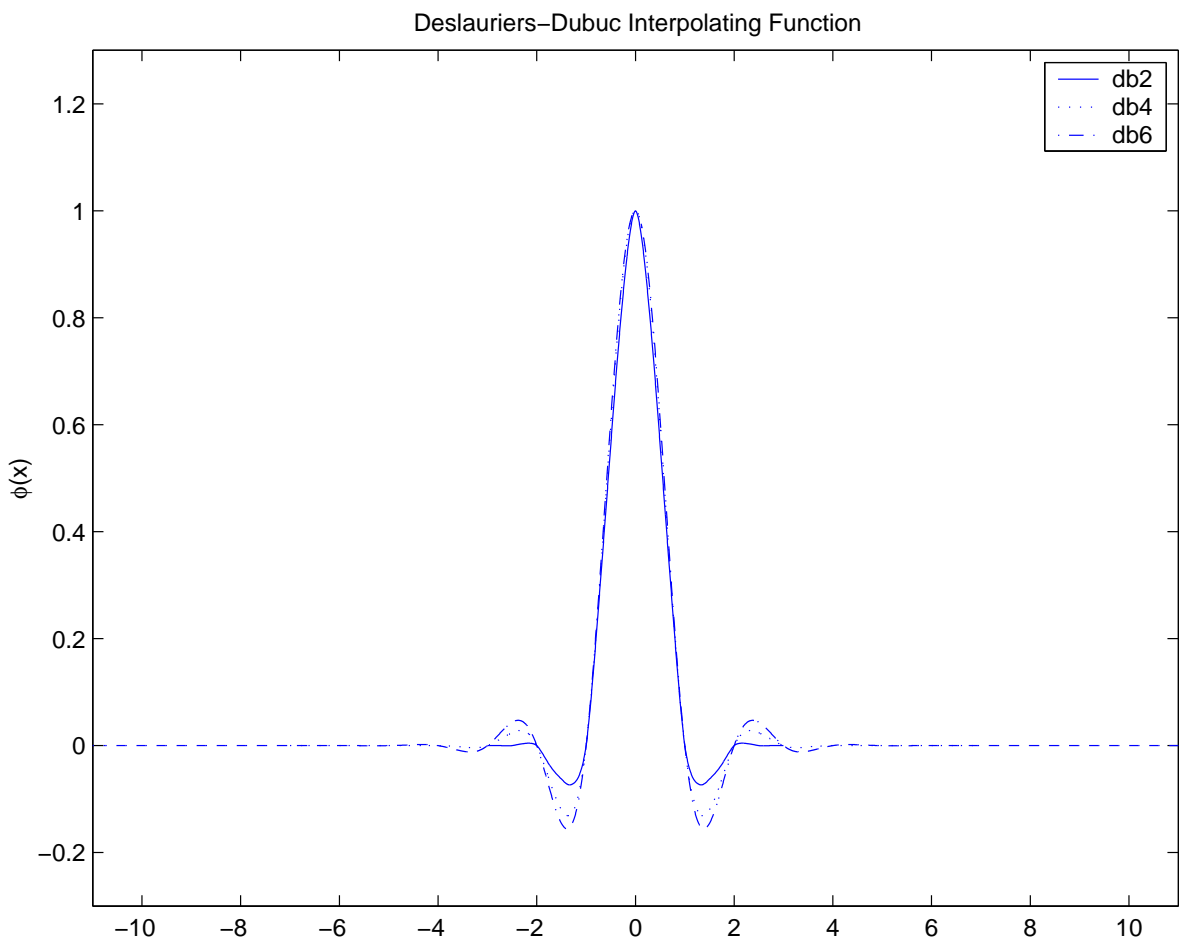

Figure 6: Deslauriers-Dubuc compactly supported interpolating functions of order 3,7 and 11 . They are autocorrelation functions of Daubechies compactly supported scaling function of order 2, 4 and 6 , respectively.

where $L s=2 p-1$, the effective support of the basis function $\phi(x)$. Similarly, equation 34 is then discretized as

$$
\tau_{i^{\prime}+1 / 2 ; n^{\prime}+1}^{x x}=\tau_{i^{\prime}+1 / 2 ; n^{\prime}}^{x x}+c_{11}^{i^{\prime}+1 / 2} \frac{\Delta t}{\Delta x} \sum_{l=0}^{L_{s}-1} a(l)\left(v_{i^{\prime}+l ; n^{\prime}+1 / 2}^{x}-v_{i^{\prime}+1-l ; n^{\prime}+1 / 2}^{x}\right)
$$

Until now, the wavelet-based formulation of FDTD for the 1-D wave equation is achieved (equations 38 and 39). To compare with conventional Taylor-expansion based FDTD schemes of 2nd, 4th, 6th and 8th order, the 1-D wave equation is discretized using 2nd, 4th, 6th and 8th order center differencing schemes for spatial derivatives and 2nd order center differencing schemes for temporal derivatives.

$$
\begin{aligned}
v_{i ; n+1 / 2}^{x} & =v_{i ; n-1 / 2}^{x}+\frac{\Delta t}{\Delta x \rho_{i}}\left[\eta_{1}\left(\tau_{i+1 / 2 ; n}^{x x}-\tau_{i-1 / 2 ; n}^{x x}\right)\right. \\
& +\eta_{2}\left(\tau_{i+3 / 2 ; n}^{x x}-\tau_{i-3 / 2 ; n}^{x x}\right) \\
& +\eta_{3}\left(\tau_{i+5 / 2 ; n}^{x x}-\tau_{i-5 / 2 ; n}^{x x}\right) \\
& \left.+\eta_{4}\left(\tau_{i+7 / 2 ; n}^{x x}-\tau_{i-7 / 2 ; n}^{x x}\right)\right] \\
\tau_{i+1 / 2 ; n+1}^{x x} & =\tau_{i+1 / 2 ; n}^{x x}+c_{11}^{i+1 / 2} \frac{\Delta t}{\Delta x}\left[\eta_{1}\left(v_{i+1 ; n+1 / 2}^{x}-v_{i ; n+1 / 2}^{x}\right)\right. \\
& +\eta_{2}\left(v_{i+2 ; n+1 / 2}^{x}-v_{i-1 ; n+1 / 2}^{x}\right) \\
& +\eta_{3}\left(v_{i+3 ; n+1 / 2}^{x}-v_{i-2 ; n+1 / 2}^{x}\right) \\
& +\eta_{4}\left(v_{i+4 ; n+1 / 2}^{x}-v_{i-3 ; n+1 / 2}^{x}\right)
\end{aligned}
$$

Coefficients $\eta_{1}, \eta_{2}, \eta_{3}$ and $\eta_{4}$ are listed in table 4.2.1 for FDTD schemes of order 2, 4, 6 and 8 . 


\begin{tabular}{|c|c|c|c|c|}
\hline & 2nd order & 4th order & 6th order & 8th order \\
\hline$\eta_{1}$ & 1 & $9 / 8$ & $75 / 64$ & $1225 / 1024$ \\
$\eta_{2}$ & & $-1 / 24$ & $-25 / 384$ & $-245 / 3072$ \\
$\eta_{3}$ & & & $3 / 640$ & $49 / 5120$ \\
$\eta_{4}$ & & & & $-5 / 7168$ \\
\hline discretization error & $O\left(\Delta x^{2}\right)$ & $O\left(\Delta x^{4}\right)$ & $O\left(\Delta x^{6}\right)$ & $O\left(\Delta x^{8}\right)$ \\
\hline
\end{tabular}

Table 3: Coefficients of 2nd, 4th, 6th and 8th order finite difference.

\subsubsection{Numerical Dispersion and Stability Condition}

Like any conventional FDTD scheme, due to the discretization in space and time, the wavelet based FDTD scheme exhibits deviations from the desired linear dispersion behavior. If numerical errors increase over time iteration, the FDTD scheme is not stable. To apply an FDTD algorithm efficiently without losing too much of accuracy, it is important to analyze numerical dispersion and stability condition.

Suppose a plane wave $e^{i(\omega t-k x)}$ is propagating in the medium, it must satisfy the wave equations 33 and 34 ; that leads to the linear dispersion relation,

$$
k^{2}=\left(\frac{\omega}{V_{p}}\right)^{2}
$$

where the compressional velocity of the medium $V_{p}$, equals to $\sqrt{c_{11} / \rho}$. Let $\hat{\omega}$ denotes the numerical angular frequency for a given wave number $k$, and $V_{p}^{\text {num }}$ denotes the numerical compressional velocity, the ratio of numerical versus true compressional velocity is defined as $q_{p}$, i.e. $q_{p}=V_{p}^{\text {num }} / V_{p}$. Let $\xi=V_{p} \frac{\Delta t}{\Delta x}$ and $H=\frac{\Delta x}{\lambda_{w}}$, where the wavelength $\lambda_{w}$, equals to $\frac{2 \pi}{k}$. $1 / H$ represents the number of grid points per wavelength. It shows in the later part of this section that $\xi$ controls the stability, while $H$ controls the numerical dispersion in the FDTD computation. Substituting the plane wave solution which is proportional to $e^{i(\hat{\omega} t-k x)}$ to the difference equations 38 and 39, yields the dispersion relation of the wavelet based FDTD scheme,

$$
\frac{1}{\xi}=\frac{\sum_{l=0}^{L s-1} a(l) \sin (2 l+1) \pi H}{\sin \pi H \xi q_{p}}
$$

When $\Delta x \rightarrow 0, H \rightarrow 0$, then $\sin (2 l+1) \pi H \sim(2 l+1) \pi H$ and $\sin \pi H \xi q_{p} \sim \pi H \xi q_{p}$, equation 43 produces the linear dispersion relation (equation 42 ) and $V_{p}^{\text {num }}=V_{p}$.

Solving for $q_{p}$, equation 43 yields

$$
q_{p}=\frac{1}{\pi H \xi} \sin ^{-1}\left\{\xi \sum_{l=0}^{L_{s}-1} a(l) \sin [(2 l+1) \pi H]\right\}
$$

The ratio between the real compressional velocity and the numerical one should remain real for a stable FDTD scheme. That requires

$$
\xi<\frac{1}{\left|\sum_{l=0}^{L_{s}-1} a(l) \sin [(2 l+1) \pi H]\right|}<\frac{1}{\sum_{l=0}^{L_{s}-1}|a(l)|}=\xi_{\max }
$$

Equation 45 is usually called the stability condition. Similarly, applying the plane wave solution to the difference equations 40 and 41 leads to the numerical dispersion relation of conventional FDTD scheme of 
the 2nd, 4th, 6th and 8 th order.

$$
\begin{aligned}
\text { 2nd order FDTD: } \frac{1}{\xi} & =\frac{\sin \pi H}{\sin \pi H \xi q_{p}} \\
\text { 4th order FDTD: } \frac{1}{\xi} & =\frac{\eta_{1} \sin \pi H+\eta_{2} \sin 3 \pi H}{\sin \pi H \xi q_{p}} \\
\text { 6th order FDTD: } \frac{1}{\xi} & =\frac{\eta_{1} \sin \pi H+\eta_{2} \sin 3 \pi H+\eta_{3} \sin 5 \pi H}{\sin \pi H \xi q_{p}} \\
\text { 8th order FDTD: } \frac{1}{\xi} & =\frac{\eta_{1} \sin \pi H+\eta_{2} \sin 3 \pi H+\eta_{3} \sin 5 \pi H+\eta_{4} \sin 7 \pi H}{\sin \pi H \xi q_{p}}
\end{aligned}
$$

From the numerical dispersion relations, we may also obtain stability conditions for corresponding conventional FDTD schemes.

$$
\xi<\frac{1}{\left|\eta_{1}\right|+\left|\eta_{2}\right|+\left|\eta_{3}\right|+\left|\eta_{4}\right|}=\xi_{\max }
$$

where $\eta_{1}, \eta_{2}, \eta_{3}$ and $\eta_{4}$ are listed in table 4.2.1.

Figures 8- 14 illustrate numerical errors in the phase velocity produced by wavelet-based and conventional FDTD schemes for various stable time steps $\Delta t$. A general trend shows that for a given accuracy, the waveletbased FDTD using 7th order Delauriers-Dubuc interpolating function, the autocorrelation function of the 4th order Daubechies scaling function, is more efficient as it requires coarser griding, specifically for coarser time steps $\left(\xi=\xi_{\max }, \xi=0.8 \xi_{\max }\right.$ and $\left.\xi=0.6 \xi_{\max }\right)$.

A 1-D FDTD algorithm that can switch between the 2nd, 4th, 6th, 8th and the wavelet-based schemes is used to test the reflection and transmission at a sharp boundary. The theoretical reflection and transmission coefficients at the sharp boundary should be $1 / 3$ and $2 / 3$, repectively, independent of frequency. Figures 15 and 16 show the transmission coefficients computed from various FDTD algorithms as a function of frequency. Figures 17 and 18 show the reflection coefficients. In all cases, the wavelet-based scheme produce more accurate results. Figure 7 shows the spectrum of the incidence wave for all aforementioned FDTD schems.

\subsubsection{Discretization of 3-D Wave Equation in Transformed Domain}

As the first step to study wave propagations in the LWD situation, we focus our interest on anisotropy and radial heterogeneity. The borehole and its surrounding formation are assumed invariant in the axial $(\mathrm{z})$ direction, and that allows us to Fourier transform the wave equations from the $(x, y, z, t)$ domain into the $\left(x, y, k_{z}, t\right)$ domain. The finite difference solution of the wave equation is obtained in the $\left(x, y, k_{z}, t\right)$ domain and transformed back to the $(x, y, z, t)$ domain in the end. The derivative respect to $z, \partial_{z}$, is then replaced by $-i k_{z}$ (equation 53), instead of using finite difference approximation. Because only $\partial_{x}$ and $\partial_{y}$ are computed using finite difference, this type of solution is called 2.5 dimensional solution. As less grid points are needed in the $k_{z}$ domain than in the $z$ domain, the 2.5D FDTD results a significant reduction in memory requirement. Since solutions for each $k_{z}$ are independent, efficient parallelization can be achieved trivially. The 3-D wave equations in the transformed domain are discretized in a 2D staggered-grid (Figure 19). To avoid confusion in notations, Fourier transform of $\mathrm{z}$ and its inverse transform are explicitly written in equations 51 and 52.

$$
\begin{array}{r}
\tilde{f}\left(k_{z}\right)=\int_{-\infty}^{+\infty} f(z) e^{i k_{z} z} d k_{z} \\
f(z)=\frac{1}{2 \pi} \int_{-\infty}^{+\infty} \tilde{f}\left(k_{z}\right) e^{-i k_{z} z} d z
\end{array}
$$

$$
\text { Fourier Transform }\left\{\partial_{z} f(x, y, z)\right\}=-i k_{z} \tilde{f}\left(x, y, k_{z}\right)
$$

Similar to the 1-D case, the wave equations are discretized using wavelet-based, 2nd, 4th, 6th and 8th order finite difference. Referring to equations $38,39,40$ and 41 , we define difference operators $\left.D_{x} \tilde{f}\right|_{m, j, k}$ 


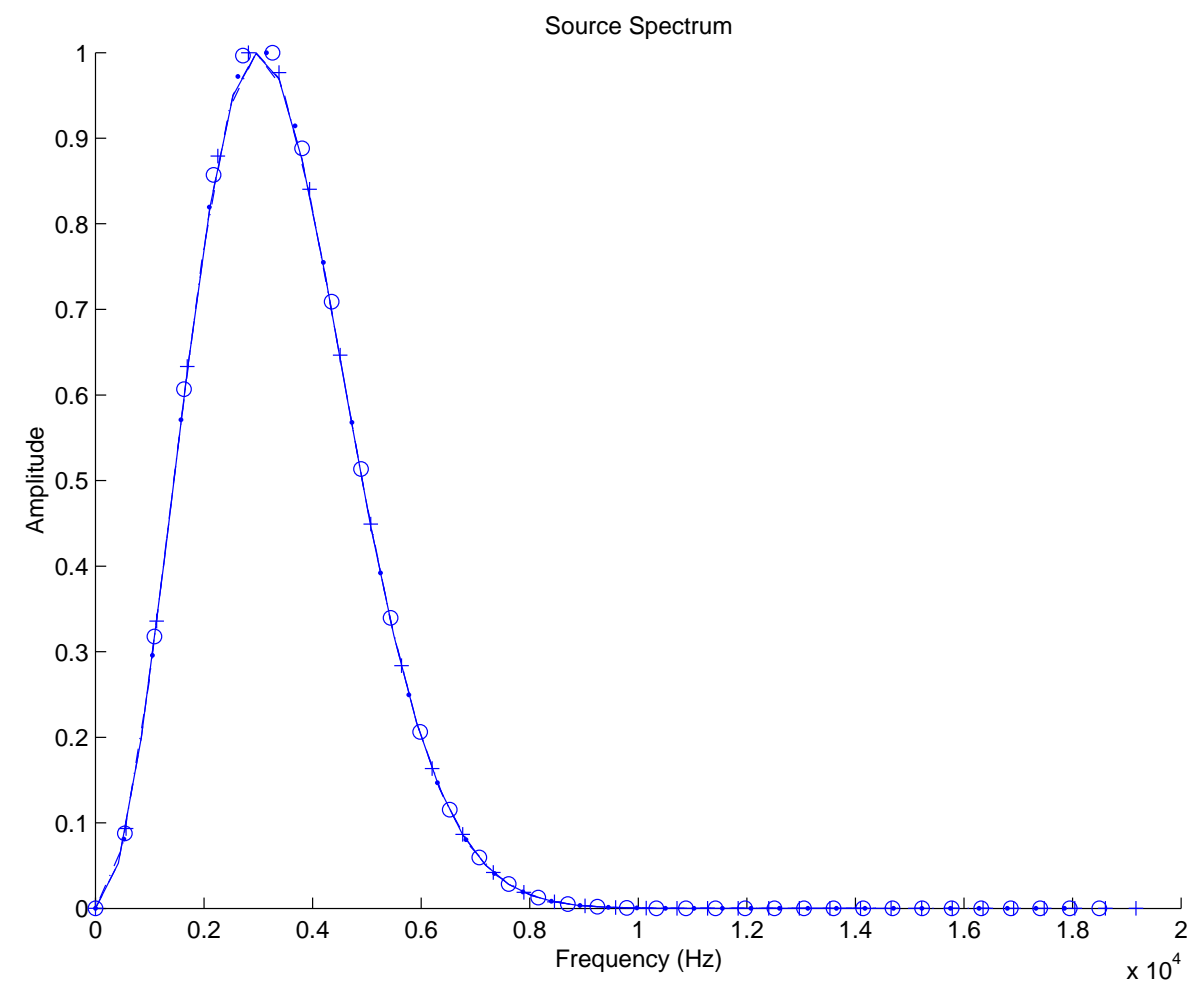

Figure 7: The spectrum of the 1-D incidence wave before it hits the sharp boundary. For all FDTD schemes, 2nd, 4th, 6th, 8th order and the wavelet-based, no numerical dispersion is observed.

and $\left.D_{y} \tilde{f}\right|_{m, j, k}$, representing the numerical evaluations at location $\left(m \Delta x, j \Delta y, k \Delta k_{z}\right)$, of the first order derivatives of $\tilde{f}$ with respect to $x$ and $y$,

$$
\begin{aligned}
& \left.D_{x} \tilde{f}\right|_{m, j, k}=\frac{1}{\Delta x} \sum_{l=0}^{L_{s}-1} h(l)\left(\tilde{f}_{m-1 / 2+l, j, k}-\tilde{f}_{m+1 / 2-l, j, k}\right) \\
& \left.D_{y} \tilde{f}\right|_{m, j, k}=\frac{1}{\Delta y} \sum_{l=0}^{L_{s}-1} h(l)\left(\tilde{f}_{m, j-1 / 2+l, k}-\tilde{f}_{m, j+1 / 2-l, k}\right)
\end{aligned}
$$

where $h(l)=a(l)$ for wavelet-based finite difference approximation, and for the 2nd, 4th, 6th and 8th order finite difference, $h(0)=0, h(p)=\eta_{p}$ (for $\left.p=1,2,3,4\right)$. In the stretching coordinate,

$$
\begin{aligned}
\left.D_{\tilde{x}} \tilde{f}\right|_{m, j, k} & =\frac{1}{\epsilon^{1}(m,:) \Delta \tilde{x}} \sum_{l=0}^{L_{s}-1} h(l)\left(\tilde{f}_{m-1 / 2+l, j, k}-\tilde{f}_{m+1 / 2-l, j, k}\right) \\
\left.D_{\tilde{y}} \tilde{f}\right|_{m, j, k} & =\frac{1}{\epsilon^{2}(:, j) \Delta \tilde{y}} \sum_{l=0}^{L_{s}-1} h(l)\left(\tilde{f}_{m, j-1 / 2+l, k}-\tilde{f}_{m, j+1 / 2-l, k}\right)
\end{aligned}
$$

where $\epsilon^{1}(m,:)$ represents discretized $x$ direction stretching function for all possible values of $j$.

Equation 13, the governing equation of the conservation of momentum in both the computational domain and the PML in the transformed coordinate, is Fourier transformed into $k_{z}$ domain and discretized. When $A=1$ and $B=0$, it reduces to the computational domain solution. Let $\Lambda=\rho A$ and $X=\rho B$, discretizing 


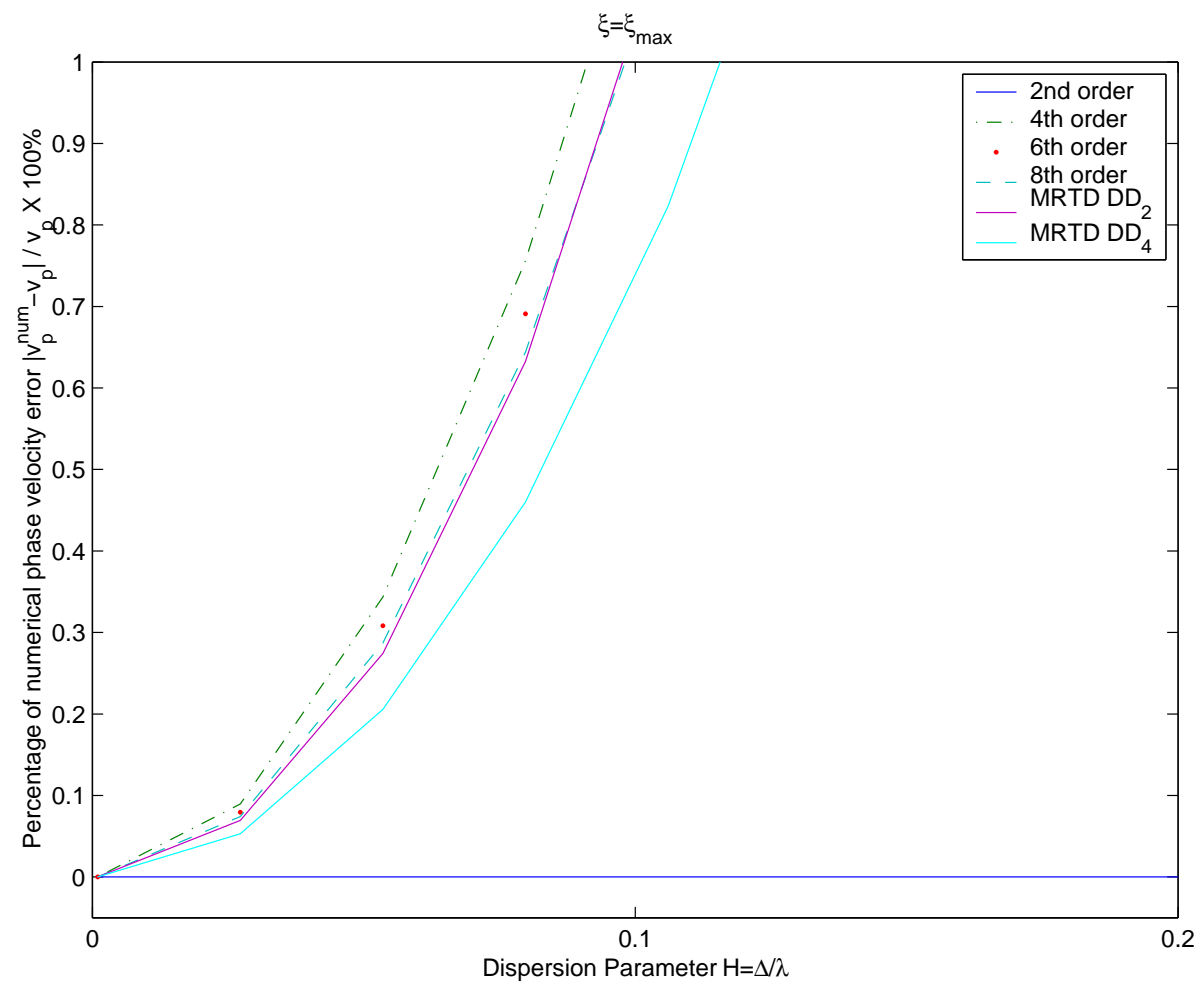

Figure 8: Numerical errors in the phase velocity with respect to $H . \Delta t=\xi_{\max } \frac{\Delta x}{V_{p}}$. Note that for the 2nd order FDTD,$\Delta t=\frac{\Delta x}{V_{p}}$, which is the so-called magic step relation in which situation the 2nd order FDTD can produce the exact solution for a single phased wave (pure compressional or shear phase) propagating in a homogeneous medium.

the $\tilde{x}$ direction component of equation 13 yields,

$$
\begin{aligned}
& \Lambda_{m, j+1 / 2} \frac{\tilde{v}_{m, j+1 / 2, k ; n+1 / 2}^{x}-\tilde{v}_{m, j+1 / 2, k ; n-1 / 2}^{x}}{\Delta t}+X_{m, j+1 / 2} \frac{\tilde{v}_{m, j+1 / 2, k ; n+1 / 2}^{x}+\tilde{v}_{m, j+1 / 2, k ; n-1 / 2}^{x}}{2} \\
= & \left.\left.D_{\tilde{x}} \tilde{\tau}^{x x}\right|_{m, j+1 / 2, k ; n}+\left.D_{\tilde{y}} \tilde{\tau}^{y x}\right|_{m, j+1 / 2, k ; n}-i k \Delta k_{z} \tilde{\tau}_{m, j+1 / 2, k ; n}^{z x}\right\} .
\end{aligned}
$$

Solving for $\tilde{v}_{m, j+1 / 2, k ; n+1 / 2}^{x}$ from equation 58 , we obtain the time iteration formula (equation 59) to update $\tilde{v}^{x}\left(m \Delta \tilde{x},(j+1 / 2) \Delta \tilde{y}, k \Delta k_{z}\right)$, at time step $n+1 / 2$, i.e. $t=(n+1 / 2) \Delta t$, from their previous time step values. Similarly, particle velocities , $\tilde{v}^{y}(m+1 / 2, j, k)$ and $\tilde{v}^{z}(m+1 / 2, j+1 / 2, k)$ can be updated from their previous time step values by equations 60 and 61 , respectively.

$$
\begin{aligned}
\tilde{v}_{m, j+1 / 2, k ; n+1 / 2}^{x} & =\frac{1}{\Psi_{m, j+1 / 2}}\left\{\left(\frac{\Lambda_{m, j+1 / 2}}{\Delta t}-\frac{X_{m, j+1 / 2}}{2}\right) \tilde{v}_{m, j+1 / 2, k ; n-1 / 2}^{x}\right. \\
& +\left.D_{\tilde{x}} \tilde{\tau}^{x x}\right|_{m, j+1 / 2, k ; n}+\left.D_{\tilde{y}} \tilde{\tau}^{y x}\right|_{m, j+1 / 2, k ; n} \\
& \left.-i k \Delta k_{z} \tilde{\tau}_{m, j+1 / 2, k ; n}^{z x}\right\} \\
\tilde{v}_{m+1 / 2, j, k ; n+1 / 2}^{y} & =\frac{1}{\Psi_{m+1 / 2, j}}\left\{\left(\frac{\Lambda_{m+1 / 2, j}}{\Delta t}-\frac{X_{m+1 / 2, j}}{2}\right)\right) \tilde{v}_{m+1 / 2, j, k ; n-1 / 2}^{y} \\
& +\left.D_{\tilde{x}} \tilde{\tau}^{x y}\right|_{m+1 / 2, j, k ; n}+\left.D_{\tilde{y}} \tilde{\tau}^{y y}\right|_{m+1 / 2, j, k ; n} \\
& -i k \Delta k_{z} \tilde{\tau}_{m+1 / 2, j, k ; n}^{z y}
\end{aligned}
$$




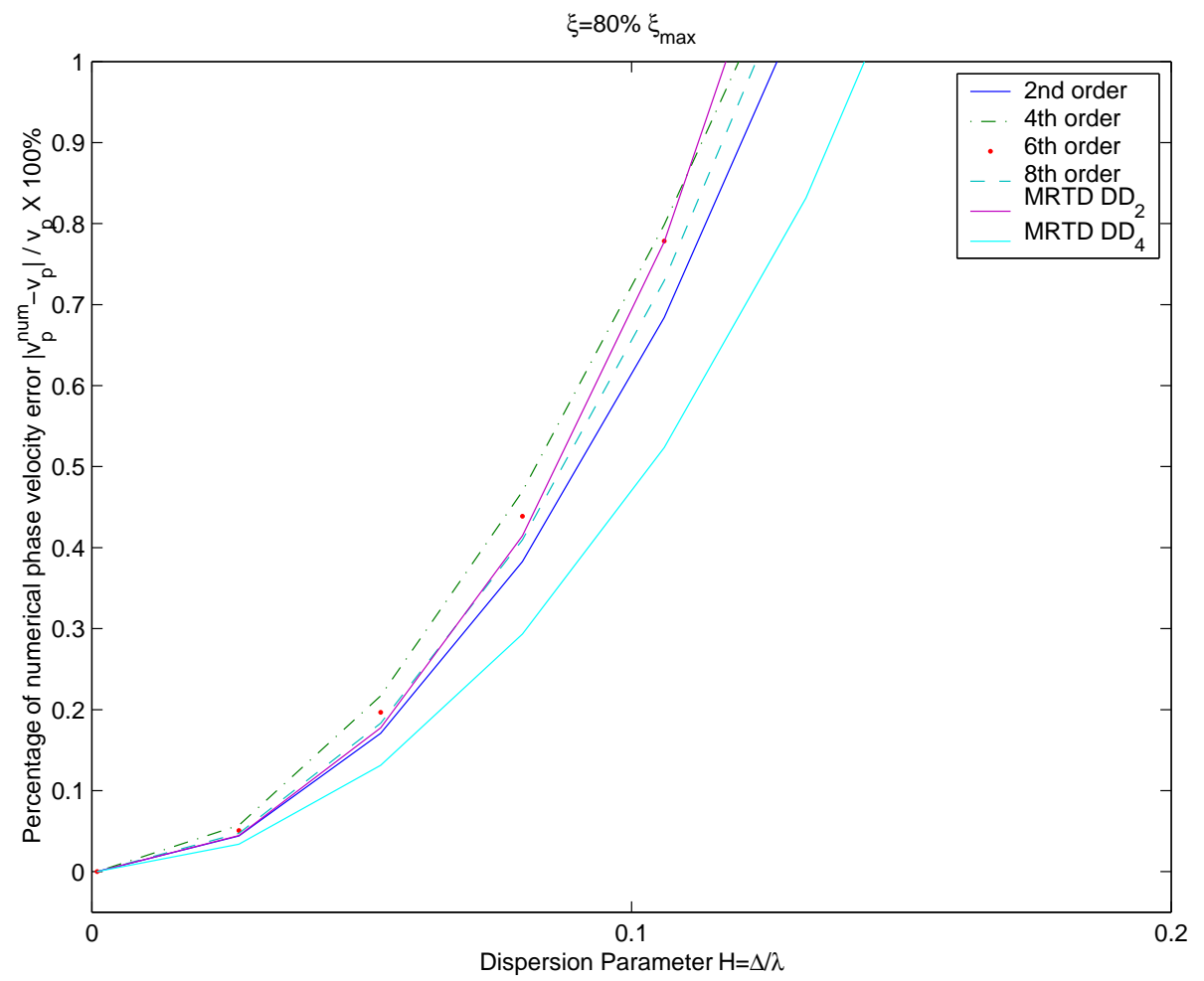

Figure 9: Numerical errors in the phase velocity with respect to $H . \Delta t=0.8 \xi_{\max } \frac{\Delta x}{V_{p}}$.

$$
\begin{aligned}
\tilde{v}_{m+1 / 2, j+1 / 2, k ; n+1 / 2}^{z} & =\frac{1}{\Psi_{m+1 / 2, j+1 / 2}}\left\{\left(\frac{\Lambda_{m+1 / 2, j+1 / 2}}{\Delta t}-\frac{X_{m+1 / 2, j+1 / 2}}{2}\right) \tilde{v}_{m+1 / 2, j+1 / 2, k ; n-1 / 2}^{z}\right. \\
& +\left.D_{\tilde{x}} \tilde{\tau}^{x z}\right|_{m+1 / 2, j+1 / 2, k ; n}+\left.D_{\tilde{y}} \tilde{\tau}^{y z}\right|_{m+1 / 2, j+1 / 2, k ; n} \\
& -i k \Delta k_{z} \tilde{\tau}_{m+1 / 2, j+1 / 2, k ; n}^{z z}
\end{aligned}
$$

where

$$
\Psi_{m, j}=\frac{\Lambda_{m, j}}{\Delta t}+\frac{X_{m, j}}{2}
$$

The constitutive relation of the PML, equations 15 and 21, can reduce to that of the computational domain by simply setting $\zeta_{1}, \zeta_{2}$ and $\zeta_{3}$ to zeros. However, the extra tensor $\overline{\bar{E}}^{\mathrm{PML}}$ requires 6 more variables, which is not necessary in the computational domain; therefore, equation 5 is used as the constitutive relation for the computational domain. Discretizing equation 5 in the stretching coordinate leads to the time iteration equations to update each stress component.

$$
\begin{aligned}
\tilde{\tau}_{m+1 / 2, j+1 / 2, k ; n+1}^{x x} & =\tilde{\tau}_{m+1 / 2, j+1 / 2, k ; n}^{x x} \\
& +\Delta t\left(\left.d_{m+1 / 2, j+1 / 2}^{11} D_{\tilde{x}} \tilde{v}^{x}\right|_{m+1 / 2, j+1 / 2, k ; n+1 / 2}\right. \\
& +\left.d_{m+1 / 2, j+1 / 2}^{12} D_{\tilde{y}} \tilde{v}^{y}\right|_{m+1 / 2, j+1 / 2, k ; n+1 / 2} \\
& \left.-d_{m+1 / 2, j+1 / 2}^{13} i k \Delta k_{z} v_{m+1 / 2, j+1 / 2, k ; n+1 / 2}^{z}\right) \\
\tilde{\tau}_{m+1 / 2, j+1 / 2, k ; n+1}^{y y} & =\tilde{\tau}_{m+1 / 2, j+1 / 2, k ; n}^{y y} \\
& +\Delta t\left(\left.d_{m+1 / 2, j+1 / 2}^{21} D_{\tilde{x}} \tilde{v}^{x}\right|_{m+1 / 2, j+1 / 2, k ; n+1 / 2}\right. \\
& +\left.d_{m+1 / 2, j+1 / 2}^{22} D_{\tilde{y}} \tilde{v}^{y}\right|_{m+1 / 2, j+1 / 2, k ; n+1 / 2} \\
& \left.-d_{m+1 / 2, j+1 / 2}^{23} i k \Delta k_{z} v_{m+1 / 2, j+1 / 2, k ; n+1 / 2}^{z}\right)
\end{aligned}
$$




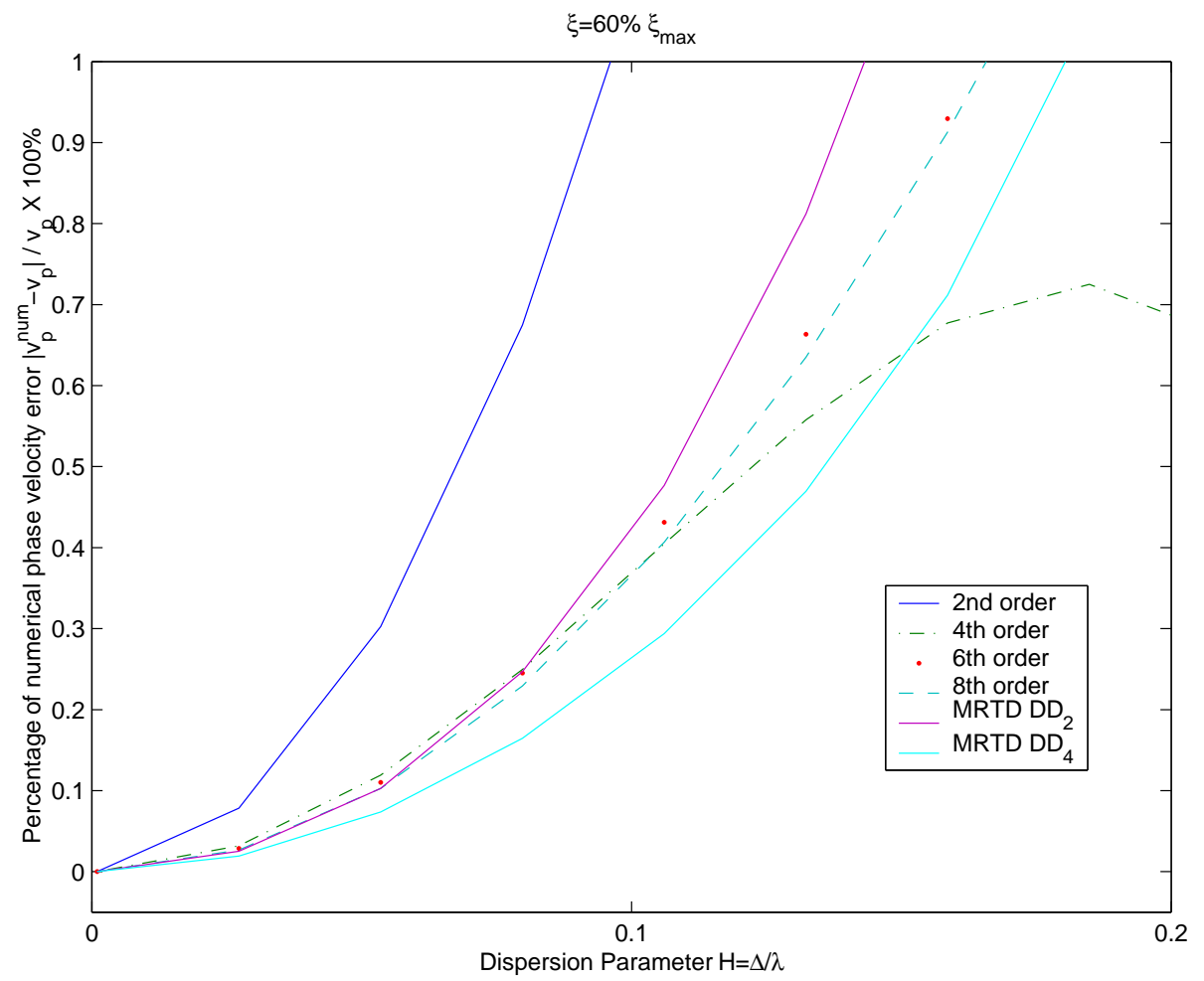

Figure 10: Numerical errors in the phase velocity with respect to $H . \Delta t=0.6 \xi_{\max } \frac{\Delta x}{V_{p}}$.

$$
\begin{aligned}
& \tilde{\tau}_{m+1 / 2, j+1 / 2, k ; n+1}^{z z}=\tilde{\tau}_{m+1 / 2, j+1 / 2, k ; n}^{z z} \\
& +\Delta t\left(\left.d_{m+1 / 2, j+1 / 2}^{31} D_{\tilde{x}} \tilde{v}^{x}\right|_{m+1 / 2, j+1 / 2, k ; n+1 / 2}\right. \\
& +\left.d_{m+1 / 2, j+1 / 2}^{32} D_{\tilde{y}} \tilde{v}^{y}\right|_{m+1 / 2, j+1 / 2, k ; n+1 / 2} \\
& \text { - } d_{m+1 / 2, j+1 / 2}^{33} i k \Delta k_{z} v_{m+1 / 2, j+1 / 2, k ; n+1 / 2}^{z} \text { ) } \\
& \tilde{\tau}_{m, j, k ; n+1}^{x y}=\tilde{\tau}_{m, j, k ; n}^{x y} \\
& +\Delta t\left(\left.d_{m, j}^{41} D_{\tilde{y}} \tilde{v}^{x}\right|_{m, j, k ; n+1 / 2}\right. \\
& \left.+\left.\quad d_{m+1 / 2, j+1 / 2}^{42} D_{\tilde{x}} \tilde{v}^{y}\right|_{m, j, k ; n+1 / 2}\right) \\
& \tilde{\tau}_{m, j, k ; n+1}^{y x}=\tilde{\tau}_{m, j, k ; n}^{y x} \\
& +\Delta t\left(\left.d_{m, j}^{51} D_{\tilde{y}} \tilde{v}^{x}\right|_{m, j, k ; n+1 / 2}\right. \\
& \left.+\left.d_{m+1 / 2, j+1 / 2}^{52} D_{\tilde{x}} \tilde{v}^{y}\right|_{m, j, k ; n+1 / 2}\right) \\
& \tilde{\tau}_{m, j+1 / 2, k ; n+1}^{x z}=\tilde{\tau}_{m, j+1 / 2, k ; n}^{x z} \\
& +\Delta t\left(-d_{m, j+1 / 2}^{61} i k \Delta k_{z} v_{m, j+1 / 2, k ; n+1 / 2}^{x}\right. \\
& \left.+\left.\quad d_{m, j+1 / 2}^{63} D_{\tilde{x}} \tilde{v}^{z}\right|_{m, j+1 / 2, k ; n+1 / 2}\right) \\
& \tilde{\tau}_{m, j+1 / 2, k ; n+1}^{z x}=\tilde{\tau}_{m, j+1 / 2, k ; n}^{z x} \\
& +\Delta t\left(-d_{m, j+1 / 2}^{71} i k \Delta k_{z} v_{m, j+1 / 2, k ; n+1 / 2}^{x}\right. \\
& \left.+\left.d_{m, j+1 / 2}^{73} D_{\tilde{x}} \tilde{v}^{z}\right|_{m, j+1 / 2, k ; n+1 / 2}\right)
\end{aligned}
$$




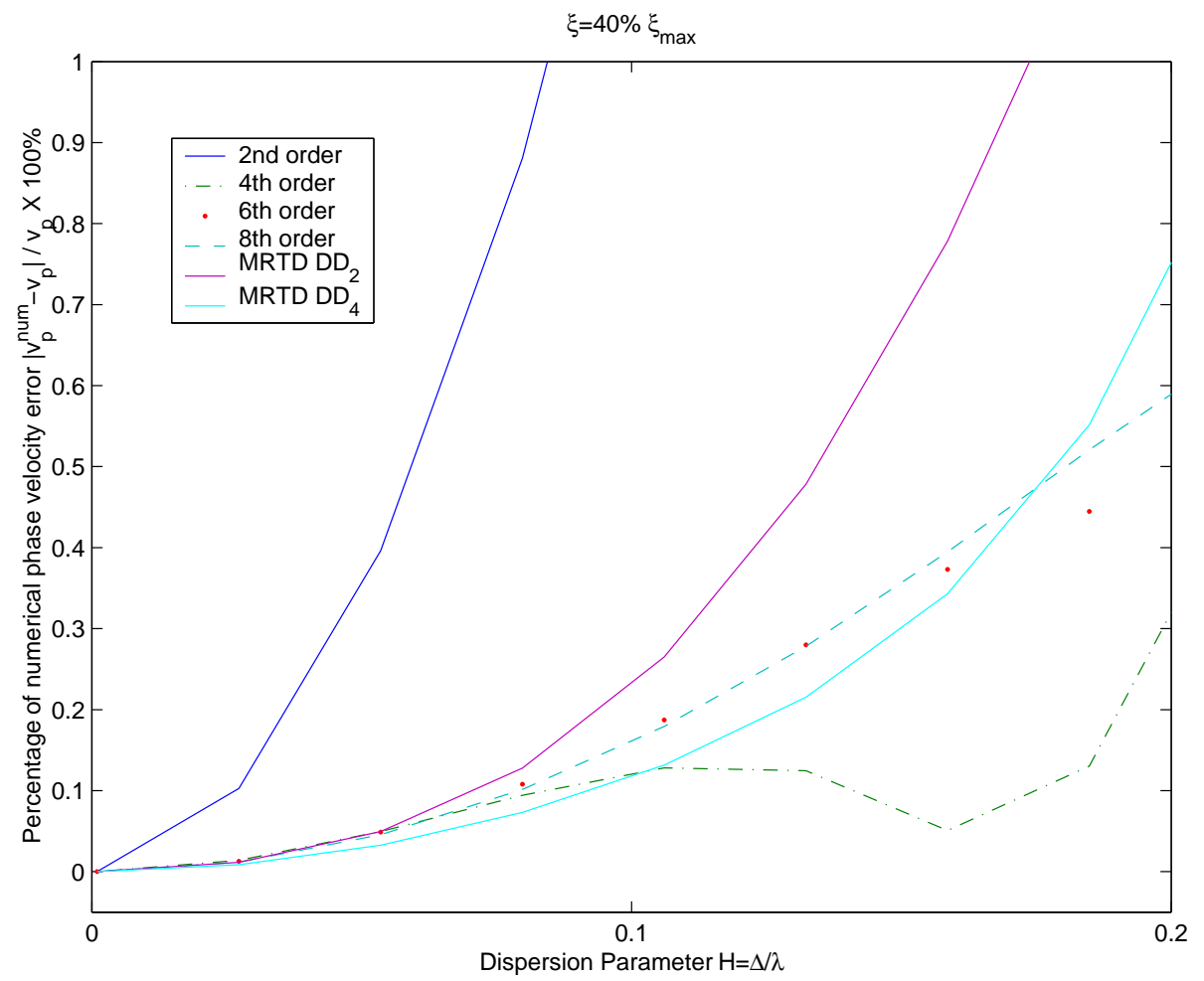

Figure 11: Numerical errors in the phase velocity with respect to $H . \Delta t=0.4 \xi_{\max } \frac{\Delta x}{V_{p}}$.

$$
\begin{aligned}
\tilde{\tau}_{m+1 / 2, j, k ; n+1}^{y z} & =\tilde{\tau}_{m+1 / 2, j, k ; n}^{y z} \\
& +\Delta t\left(-d_{m+1 / 2, j}^{82} i k \Delta k_{z} v_{m+1 / 2, j, k ; n+1 / 2}^{y}\right. \\
& \left.+\left.d_{m+1 / 2, j}^{83} D_{\tilde{y}} \tilde{v}^{z}\right|_{m+1 / 2, j, k ; n+1 / 2}\right) \\
\tilde{\tau}_{m+1 / 2, j, k ; n+1}^{z y} & =\tilde{\tau}_{m+1 / 2, j, k ; n}^{z y} \\
& +\Delta t\left(-d_{m+1 / 2, j}^{92} i k \Delta k_{z} v_{m+1 / 2, j, k ; n+1 / 2}^{y}\right. \\
& \left.+\left.d_{m+1 / 2, j}^{93} D_{\tilde{y}} \tilde{v}^{z}\right|_{m+1 / 2, j, k ; n+1 / 2}\right)
\end{aligned}
$$

When considering wave propagation in an elastic medium without residual stresses, the stress tensor is symmetric, i.e., $\tau_{\alpha \beta}=\tau_{\beta \alpha}$ and $d_{p \alpha}$ denotes the elasticity of the medium. For orthorhombic anisotropy,

$$
\left[d_{p \alpha}\right]=\left[\begin{array}{ccc}
c_{11} & c_{12} & c_{13} \\
c_{12} & c_{22} & c_{23} \\
c_{13} & c_{23} & c_{33} \\
c_{66} & c_{66} & 0 \\
c_{66} & c_{66} & 0 \\
c_{55} & 0 & c_{55} \\
c_{55} & 0 & c_{55} \\
0 & c_{44} & c_{44} \\
0 & c_{44} & c_{44}
\end{array}\right]
$$

Time iteration for stress components in the PML is updated using two steps. First, update the strain rate, $\vec{E}^{\mathrm{PML}}$, from finite difference approximations of the equation 21 ; then update the stress from difference 


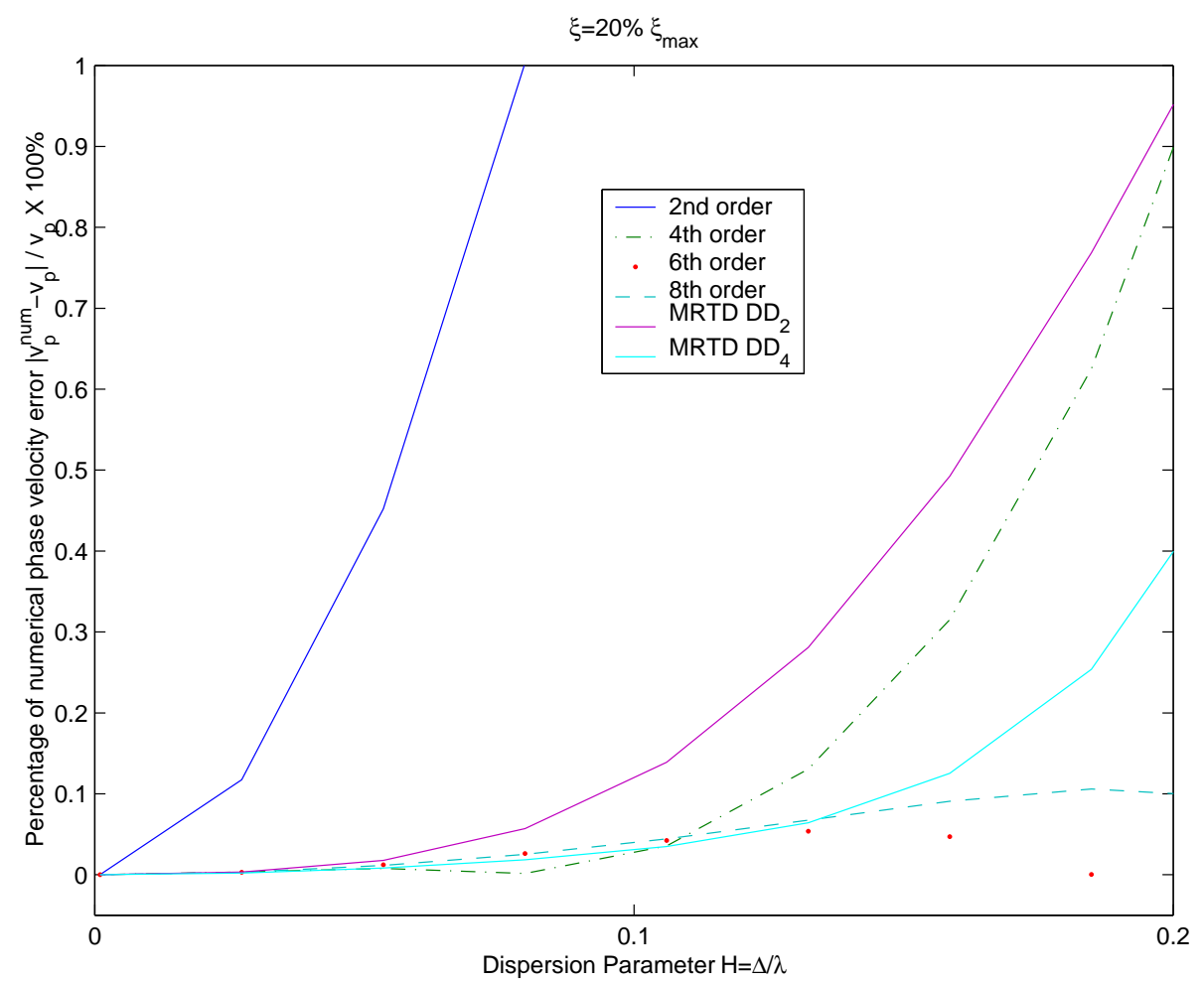

Figure 12: Numerical errors in the phase velocity with respect to $H . \Delta t=0.2 \xi_{\max } \frac{\Delta x}{V_{p}}$.

equations obtained from 15. Before applying finite difference approximations, both equations 21 and 15 are Fourier transformed into $\left(\tilde{x}, \tilde{y}, k_{z}, \mathrm{t}\right)$ domain.

$$
\begin{aligned}
\tilde{E}_{m+1 / 2, j+1 / 2, k ; n+1}^{x x} & =\frac{1}{\gamma_{1}^{-}}\left(-\gamma_{1}^{+} \tilde{E}_{m+1 / 2, j+1 / 2, k ; n}^{x x}+\left.D_{\tilde{x}} \tilde{v}^{x}\right|_{m+1 / 2, j+1 / 2, k ; n+1 / 2}\right) \\
\tilde{\tau}_{m+1 / 2, j+1 / 2, k ; n+1}^{x x}= & \frac{1}{\sigma_{1}^{+}}\left\{\sigma_{1}^{-} \tilde{\tau}_{m+1 / 2, j+1 / 2, k ; n}^{x x}+\left(d_{m+1 / 2, j+1 / 2}^{11} \hat{\tilde{E}}_{m+1 / 2, j+1 / 2, k ; 1 / 2}^{x x}\right.\right. \\
+ & \left.\left.d_{m+1 / 2, j+1 / 2}^{12} \hat{\tilde{E}}_{m+1 / 2, j+1 / 2, k ; 1 / 2}^{y y}+d_{m+1 / 2, j+1 / 2}^{13} \hat{\tilde{E}}_{m+1 / 2, j+1 / 2, k ; 1 / 2}^{z z}\right)\right\} \\
\tilde{E}_{m+1 / 2, j+1 / 2, k ; n+1}^{y y}= & \frac{1}{\gamma_{2}^{-}}\left(-\gamma_{2}^{+} \tilde{E}_{m+1 / 2, j+1 / 2, k ; n}^{y y}+\left.D_{\tilde{y}} \tilde{v}^{y}\right|_{m+1 / 2, j+1 / 2, k ; n+1 / 2}\right) \\
= & \frac{1}{\sigma_{2}^{+}}\left\{\sigma_{2}^{-} \tilde{\tau}_{m+1 / 2, j+1 / 2, k ; n}^{y y}+\left(d_{m+1 / 2, j+1 / 2}^{21} \hat{\tilde{E}}_{m+1 / 2, j+1 / 2, k ; 1 / 2}^{x x}\right.\right. \\
+ & \left.\left.d_{m+1 / 2, j+1 / 2}^{22} \hat{\tilde{E}}_{m+1 / 2, j+1 / 2, k ; 1 / 2}^{y y}+d_{m+1 / 2, j+1 / 2}^{23} \hat{\tilde{E}}_{m+1 / 2, j+1 / 2, k ; 1 / 2}^{z z}\right)\right\} \\
\tilde{\tau}_{m+1 / 2, j+1 / 2, k ; n+1}^{y y} & =\frac{1}{\gamma_{3}^{-}}\left(-\gamma_{3}^{+} \tilde{E}_{m+1 / 2, j+1 / 2, k ; n}^{z z}-\left.i k \Delta k_{z} \tilde{v}^{z}\right|_{m+1 / 2, j+1 / 2, k ; n+1 / 2}\right) \\
\tilde{E}_{m+1 / 2, j+1 / 2, k ; n+1}^{z z} & \frac{1}{\sigma_{3}^{+}}\left\{\sigma_{3}^{-} \tilde{\tau}_{m+1 / 2, j+1 / 2, k ; n}^{z z}+\left(d_{m+1 / 2, j+1 / 2}^{31} \hat{\tilde{E}}_{m+1 / 2, j+1 / 2, k ; 1 / 2}^{x x} \hat{\tilde{E}}_{m+1 / 2, j+1 / 2, k ; 1 / 2}^{z z}\right)\right\} \\
+ & d_{m+1 / 2, j+1 / 2}^{32} \hat{\tilde{E}}_{m+1 / 2, j+1 / 2, k ; 1 / 2}^{y y}+d_{m+1 / 2, j+1 / 2}^{33}
\end{aligned}
$$




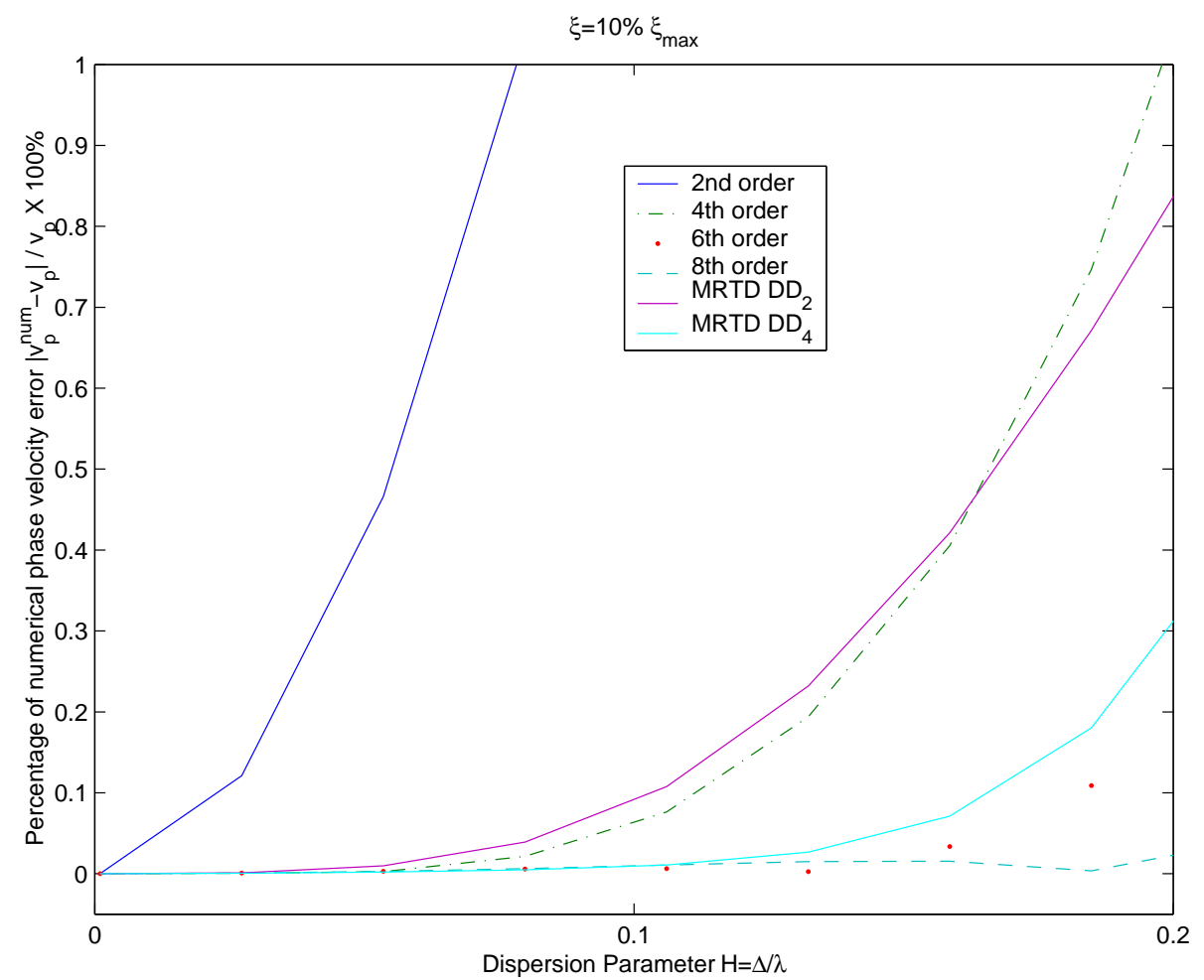

Figure 13: Numerical errors in the phase velocity with respect to $H . \Delta t=0.1 \xi_{\max } \frac{\Delta x}{V_{p}}$.

$$
\begin{aligned}
& \tilde{E}_{m, j, k ; n+1}^{x y}=\frac{1}{\gamma_{1}^{-}}\left(-\gamma_{1}^{+} \tilde{E}_{m, j, k ; n}^{x y}+\left.D_{\tilde{x}} \tilde{v}^{y}\right|_{m, j, k ; n+1 / 2}\right) \\
& \tilde{E}_{m, j, k ; n+1}^{y x}=\frac{1}{\gamma_{2}^{-}}\left(-\gamma_{2}^{+} \tilde{E}_{m, j, k ; n}^{y x}+\left.D_{\tilde{y}} \tilde{v}^{x}\right|_{m, j, k ; n+1 / 2}\right) \\
& \tilde{\tau}_{m, j, k ; n+1}^{x x}=\frac{1}{\sigma_{1}^{+}}\left\{\sigma_{1}^{-} \tilde{\tau}_{m, j, k ; n}^{x y}+d_{m, j}^{41} \hat{\tilde{E}}_{m, j, k ; 1 / 2}^{y x}\right. \\
& \left.+d_{m, j}^{42} \hat{\tilde{E}}_{m, j, k ; 1 / 2}^{x y}\right\} \\
& \tilde{\tau}_{m, j, k ; n+1}^{y x}=\frac{1}{\sigma_{2}^{+}}\left\{\sigma_{2}^{-} \tilde{\tau}_{m, j, k ; n}^{y x}+d_{m, j}^{51} \hat{\tilde{E}}_{m, j, k ; 1 / 2}^{y x}\right. \\
& \left.+\quad d_{m, j}^{52} \hat{\tilde{E}}_{m, j, k ; 1 / 2}^{x y}\right\} \\
& \tilde{E}_{m, j+1 / 2, k ; n+1}^{x z}=\frac{1}{\gamma_{1}^{-}}\left(-\gamma_{1}^{+} \tilde{E}_{m, j+1 / 2, k ; n}^{x z}+\left.D_{\tilde{x}} \tilde{v}^{z}\right|_{m, j+1 / 2, k ; n+1 / 2}\right) \\
& \tilde{E}_{m, j+1 / 2, k ; n+1}^{z x}=\frac{1}{\gamma_{3}^{-}}\left(-\gamma_{3}^{+} \tilde{E}_{m, j+1 / 2, k ; n}^{z x}-\left.i k \Delta k_{z} \tilde{v}^{x}\right|_{m, j+1 / 2, k ; n+1 / 2}\right) \\
& \tilde{\tau}_{m, j+1 / 2, k ; n+1}^{x z}=\frac{1}{\sigma_{1}^{+}}\left\{\sigma_{1}^{-} \tilde{\tau}_{m, j+1 / 2, k ; n}^{x z}+d_{m, j}^{61} \hat{\tilde{E}}_{m, j+1 / 2, k ; 1 / 2}^{z x}\right. \\
& \left.+d_{m, j}^{63} \hat{\tilde{E}}_{m, j+1 / 2, k ; 1 / 2}^{x z}\right\}
\end{aligned}
$$




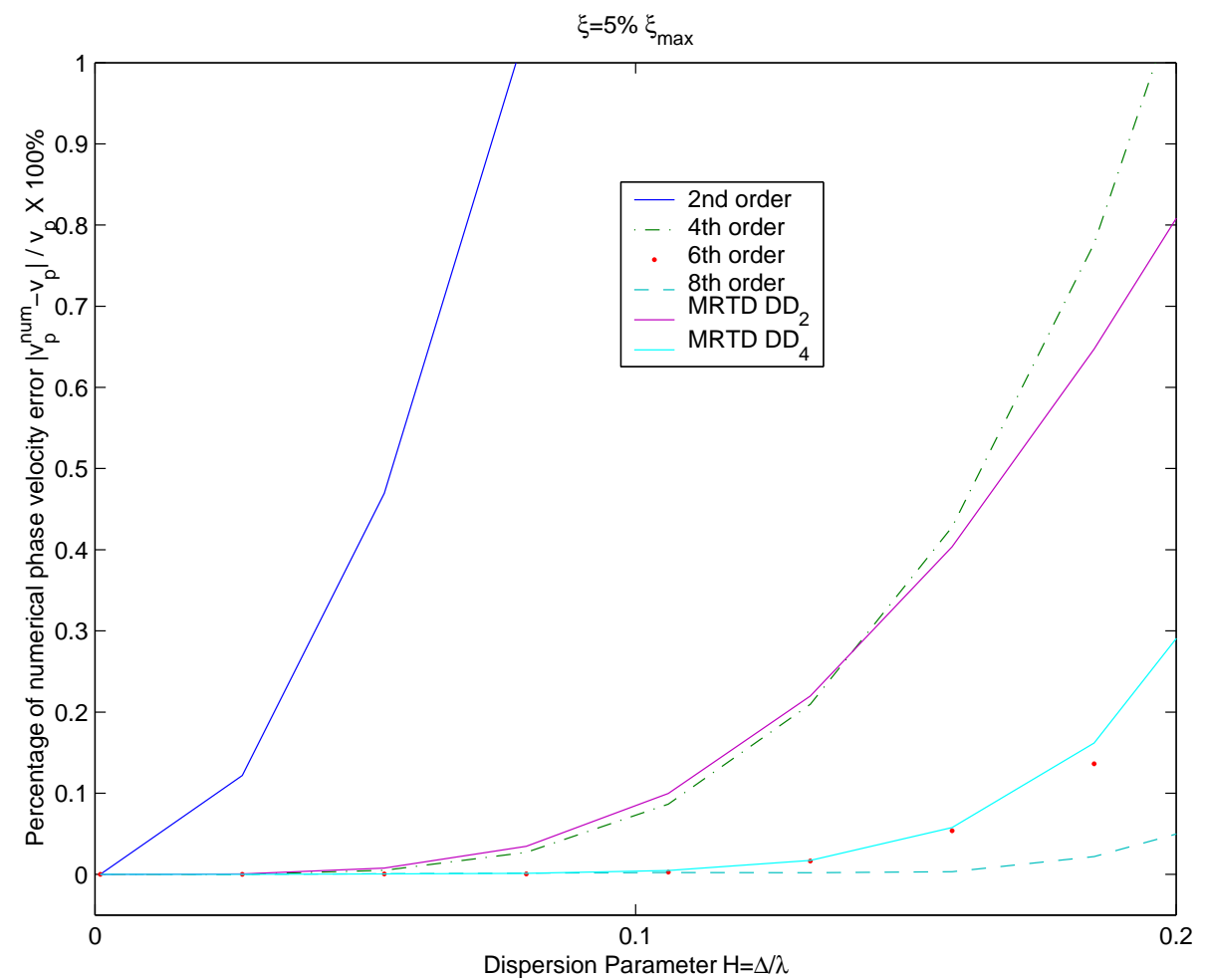

Figure 14: Numerical errors in the phase velocity with respect to $H . \Delta t=0.05 \xi_{\max } \frac{\Delta x}{V_{p}}$.

$$
\begin{aligned}
& \tilde{\tau}_{m, j+1 / 2, k ; n+1}^{z x}=\frac{1}{\sigma_{3}^{+}}\left\{\sigma_{3}^{-} \tilde{\tau}_{m, j+1 / 2, k ; n}^{z x}+d_{m, j}^{71} \hat{\tilde{E}}_{m, j+1 / 2, k ; 1 / 2}^{z x}\right. \\
&\left.+d_{m, j}^{73} \hat{\tilde{E}}_{m, j+1 / 2, k ; 1 / 2}^{x z}\right\} \\
& \tilde{E}_{m+1 / 2, j, k ; n+1}^{y z}= \frac{1}{\gamma_{2}^{-}}\left(-\gamma_{2}^{+} \tilde{E}_{m+1 / 2, j, k ; n}^{y z}+\left.D_{\tilde{y}} \tilde{v}^{z}\right|_{m+1 / 2, j, k ; n+1 / 2}\right) \\
& \tilde{E}_{m+1 / 2, j, k ; n+1}^{z y}=\frac{1}{\gamma_{3}^{-}}\left(-\gamma_{3}^{+} \tilde{E}_{m+1 / 2, j, k ; n}^{z y}-\left.i k \Delta k_{z} \tilde{v}^{y}\right|_{m+1 / 2, j, k ; n+1 / 2}\right) \\
&=\frac{1}{\sigma_{2}^{+}\left\{\sigma_{2}^{-} \tilde{\tau}_{m+1 / 2, j, k ; n}^{y z}+d_{m+1 / 2, j}^{82} \hat{\tilde{E}}_{m+1 / 2, j, k ; 1 / 2}^{z y}\right.} \\
&\left.+d_{m+1 / 2, j}^{83} \hat{\tilde{E}}_{m+1 / 2, j, k ; 1 / 2}^{y z}\right\} \\
& \tilde{\tau}_{m+1 / 2, j, k ; n+1}^{y z} \\
&+\frac{1}{\sigma_{3}^{+}}\left\{\sigma_{3}^{-} \tilde{\tau}_{m+1 / 2, j, k ; n}^{z y}+d_{m+1 / 2, j}^{92} \hat{\tilde{E}}_{m+1 / 2, j, k ; 1 / 2}^{z y} \hat{\tilde{E}}_{m+1 / 2, j, k ; 1 / 2}^{y z}\right\} \\
&+d_{m+1 / 2, j}^{93}
\end{aligned}
$$




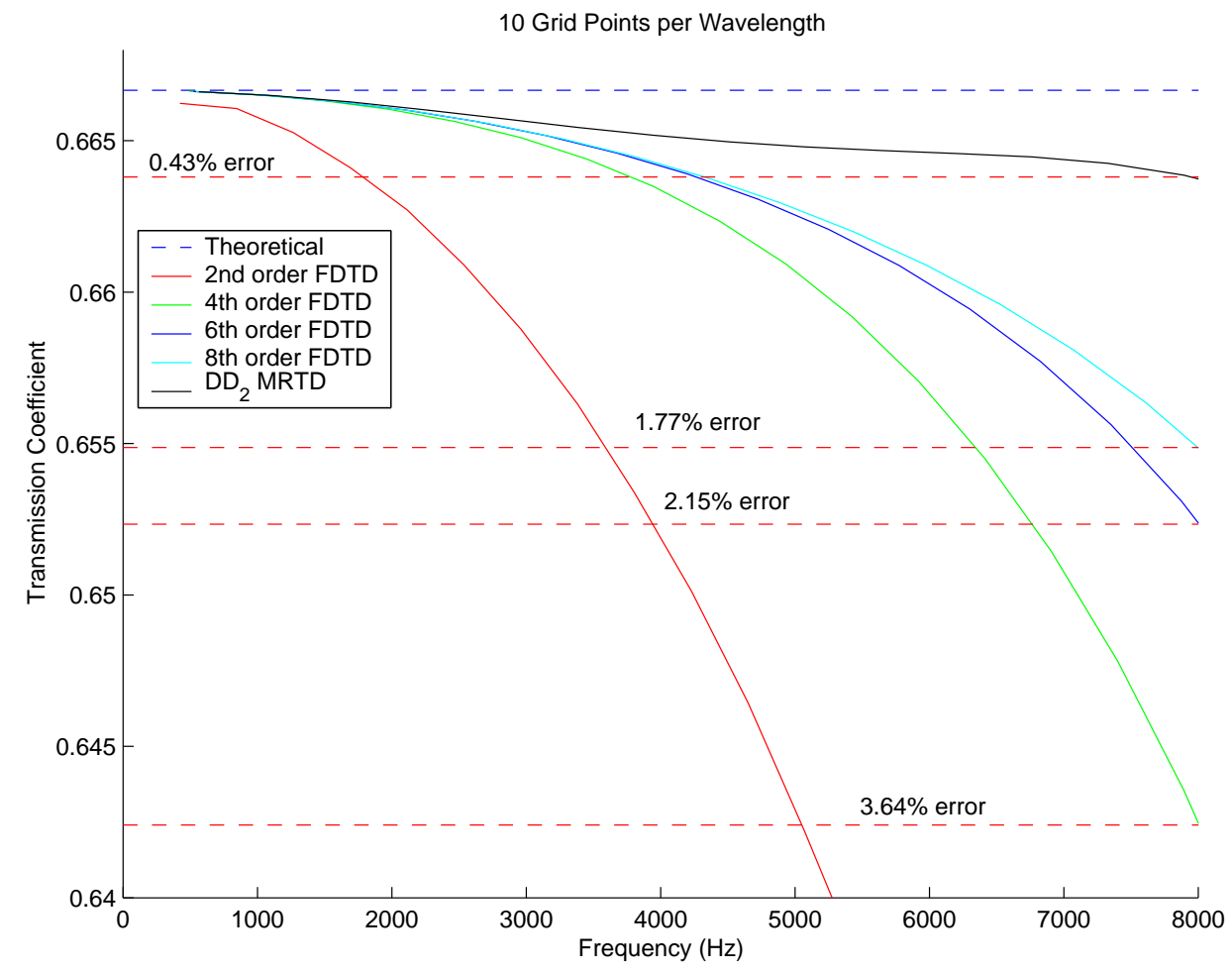

Figure 15: Transmission coefficients obtained from FDTD computations. The wavelet-based FDTD outperforms conventional FDTDs.

where

$$
\begin{aligned}
\gamma_{p}^{+} & =\frac{\zeta_{p}}{\omega \Delta t}+\frac{1}{2} \\
\gamma_{p}^{-} & =-\frac{\zeta_{p}}{\omega \Delta t}+\frac{1}{2} \\
\sigma_{p}^{+} & =\frac{R \pi^{p}}{\Delta t}+\frac{I \pi^{p}}{2} \\
\sigma_{p}^{-} & =\frac{R \pi^{p}}{\Delta t}-\frac{I \pi^{p}}{2}
\end{aligned}
$$

with $p=1,2$ and 3. $\hat{\tilde{E}}_{m, j, k ; n+1 / 2}^{\alpha \beta}$ represents the average of the strain rate component at current and previous time steps, i.e.

$$
\hat{\tilde{E}}_{m, j, k ; n+1 / 2}^{\alpha \beta}=\frac{\tilde{E}_{m, j, k ; n+1}^{\alpha \beta}+\tilde{E}_{m, j, k ; n}^{\alpha \beta}}{2}
$$

At this point, we have established finite difference solutions for the elastic wave equations in the computational domain and the PML. Because all equations are derived in the stretching coordinate, depending on the shape of stretching functions $\epsilon^{1}(\tilde{x})$ and $\epsilon^{1}(\tilde{x})$, grid size in the physical domain is variable. Following the same approach as for the 1-D case, we may obtain the stability condition for the 2.5D FDTD scheme,

$$
\xi \leq \frac{1}{\sum_{l=0}^{L_{s}-1}|h(l)|}\left(2+\frac{k_{z}^{2} \Delta^{2}}{4}\right)^{-\frac{1}{2}}
$$

For simplicity, let $\Delta x=\Delta y=\Delta$. 


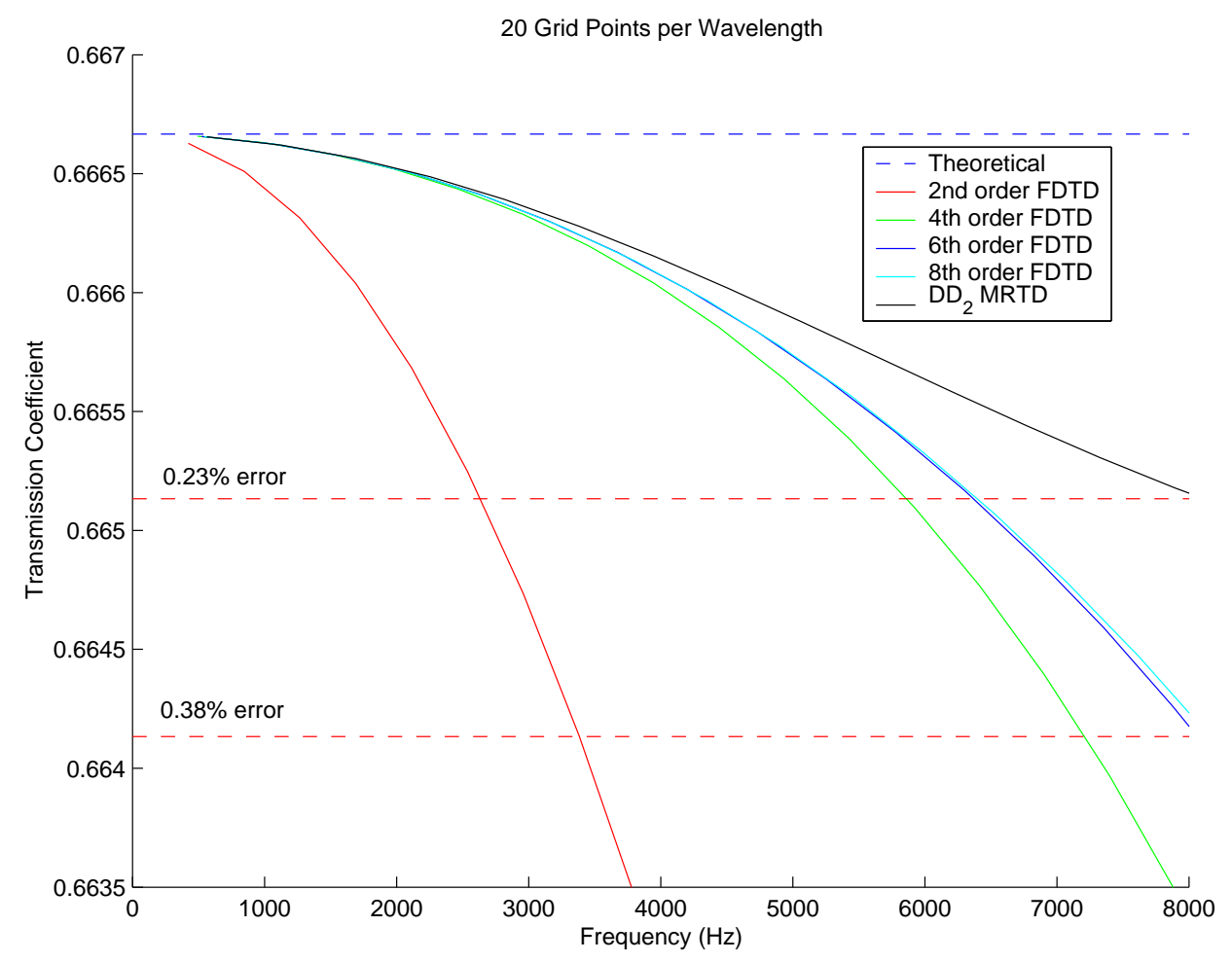

Figure 16: Transmission coefficients obtained from FDTD computations. The wavelet-based FDTD outperforms conventional FDTDs.

\section{$5 \quad$ Test of Finite Difference Method}

We implemented a staggered-grid 2.5D FDTD code with the anisotropic PML using 2nd order center difference for temporal derivatives. For spatial derivatives, we leave the option to the user, depending on their specific needs, of selecting $2 \mathrm{nd}, 4 \mathrm{th}, 6 \mathrm{th}, 8 \mathrm{th}$ and wavelet-based difference schemes. The program can take an arbitrary heterogeneous model. The grid size can be non-uniform and the user can specify where and how the grid should be stretched. The Kelly wavelet is employed as the source function (Stephen et al., 1985).

First, we test our algorithm with a point source in a homogeneous acoustic medium, and compare the results with Green's function solution. Then, we test it with a point source in a fluid-filled borehole.

\subsection{Homogeneous Acoustic Medium}

By setting the shear velocity at zero, we obtain an acoustic medium. The point source solution to the wave equation in a homogeneous acoustic medium is proportional to $g\left(t-r / V_{p}\right) / r$, where $g(t)$ is the Kelly source function, $r$ is the source-receiver distance.

The variable grid option is turned off. Wavelet-based(DD4) difference is chosen to approximate spatial derivatives. We choose $500 \mathrm{~Hz}$ as the center frequency, the compressional velocity as $3000 \mathrm{~m} / \mathrm{s}$ and the density as $2000 \mathrm{~kg} / \mathrm{m}^{3}$. The grid size is $0.5 \mathrm{~m}$, implying 12 grid points per wavelength. $k_{z}$ was chosen from 0 to $6.281 / \mathrm{m}$ with a spacing of $0.03141 / \mathrm{m}$. The source and receiver layout is shown in Figures 20 and 22 . Both analytical (Green's function solution) and the FDTD results are shown in Figure 21 and 23. The FDTD results agree with the analytical solution very well. 


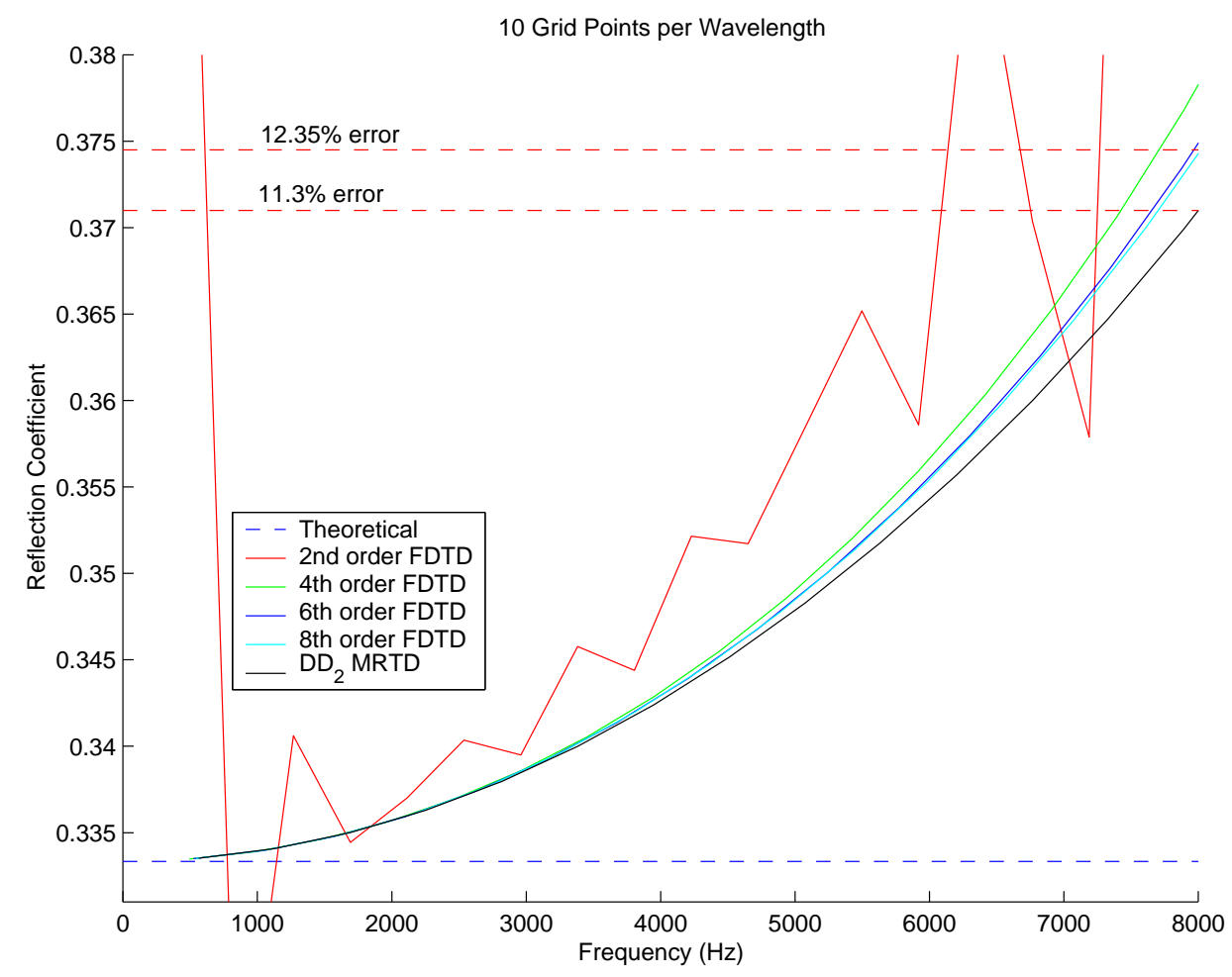

Figure 17: Reflection coefficients obtained from FDTD computations. The wavelet-based FDTD outperforms conventional FDTDs.

\subsection{Fluid-filled Borehole With Monopole Source}

We test our new algorithm with the classic acoustic logging model: a fluid-filled borehole with a monopole source at the center, as the main purpose of developing the new FDTD algorithm in this paper is to study wave propagation in a borehole.Wave propagation in a fluid-filled borehole has been well studied analytically (Biot, 1952). Physical properties of the fluid and formation are listed in table 5.2. Shown in Figure 24, the mesh is finer inside borehole and coarser in the formation. The stretching function $\epsilon^{1}(\tilde{x})=\epsilon^{2}(\tilde{y})$, is illustrated in Figure 26. A top view of the discretized borehole is shown in Figure 25. If using same grid numbers, a uniform grid would discretize the borehole less smooth and circular. Figure 27 shows the top view of the borehole discretized by a uniform grid. The source center frequency is chosen as $8 \mathrm{Khz} . k_{z}$ is chosen from 0 to $180.51 / \mathrm{m}$ with $\Delta k_{z}$ equal to $0.51 / \mathrm{m}$. 8th order differencing scheme is chosen to approximate spatial derivatives. The finest grid size is $0.007 \mathrm{~m}$, from the center of the borehole to a radial length of $0.15 \mathrm{~m}$. The coarsest grid size is $0.021 \mathrm{~m}$. The transition zone between the fine and the coarse grid is $0.1 \mathrm{~m}$.

The total grid size is $112 \times 112 \times 362$. As results in $k_{z}$ domain are independent, it is trivial to divide the model into several pieces with each piece running on one machine if a single machine does not have enough memory to hold the whole model. It took three $1.4 \mathrm{GHz}$ Pentium PC 19 hours to complete the simulation.

Figure 28 shows the waveforms received from the eight receivers. The waveforms are then subjected to the dispersion analysis, an algorithm developed at ERL (Nolte et al., 1997). The results are plotted against the theoretical curves 29. The numerical results clearly show the Stoneley mode and the first order pseudo-Rayleigh mode, agreeing with the analytical solution very well. Further semblance analysis result also clearly shows those two modal arrivals as well as the compressional arrival 30. 


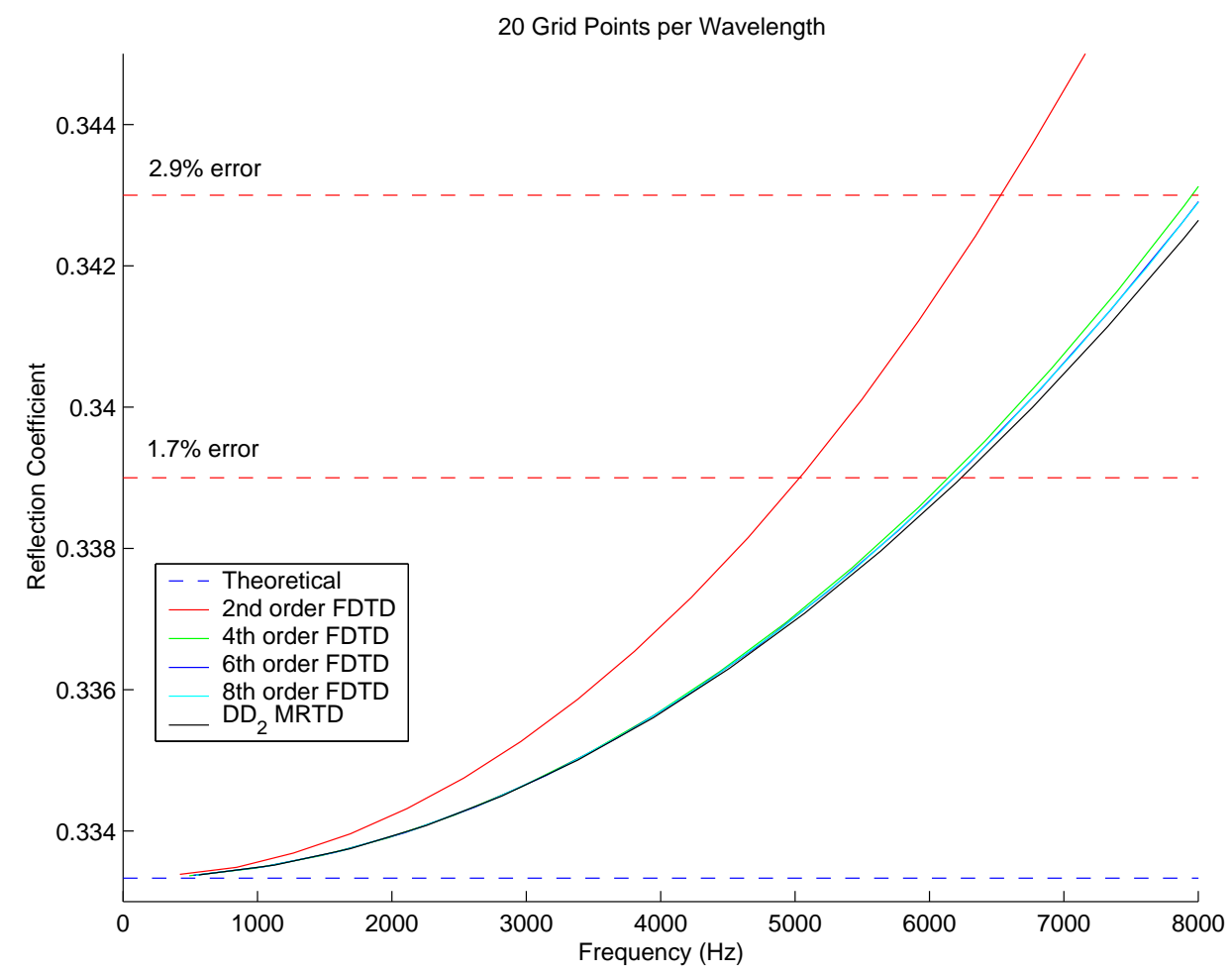

Figure 18: Reflection coefficients obtained from FDTD computations. The wavelet-based FDTD outperforms conventional FDTDs.

\section{Conclusions}

We investigated the efficiency and accuracy of the finite difference time domain scheme from three prospectives: the griding scheme, the differencing operator, and the numerical truncation scheme. A coordinate stretching approach was developed to discretize the physical space using variable grid size. Testing with a borehole model, we proved that this is stable, easy to implement, and most of all efficient and accurate. For the differencing operator, we formulated a wavelet-based differencing scheme, which exhibits linear properties, thus allowing for coarser griding. Besides improved efficiency, the wavelet-based FDTD is more accurate for reflections and transmissions at a sharp boundary. We also formulated the popular anisotropic PML in the stretching coordinate.

\section{Acknowledgments}

This work was supported by the Department of Energy Grant \#DE-FC26-02NT15346, and by the M.I.T. Borehole Acoustics and Logging Consortium, the Founding Members of the Earth Resources Laboratory at the Massachusetts Institute of Technology. 

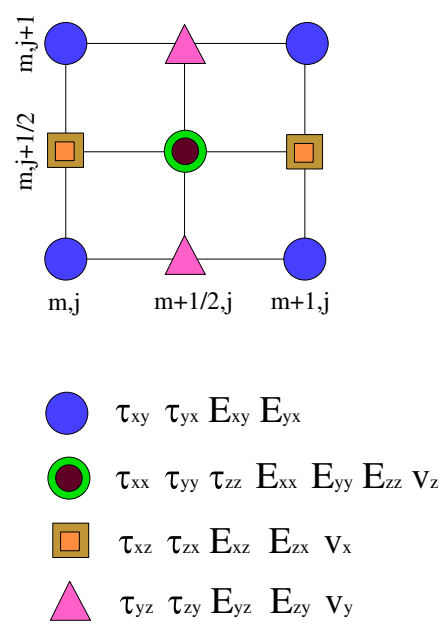

Figure 19: Schematics of staggered-grids for 2.5D schemes.

\begin{tabular}{|c|c|c|}
\hline & $\rho$ & 1000 \\
water & $V_{p}$ & 1500 \\
\hline & $\rho$ & 2000 \\
formation & $V_{p}$ & 3000 \\
& $V_{s}$ & 2000 \\
\hline borehole radius & $\mathrm{r}$ & 0.1 \\
\hline source 1st receiver offset & $z_{0}$ & 3.3528 \\
\hline receiver offset & $\mathrm{dz}$ & 0.1524 \\
\hline number of receivers & $\mathrm{nrec}$ & 8 \\
\hline
\end{tabular}

Table 4: Model parameters.

\section{References}

Alterman, Z. and Karal, F. C. (1968). Propagation of elastic waves in layered media by finite difference methods. Bulletin of the Seismological Society of America, 58:367-398.

Anthes, R. A. (1970). Numerical experiments with a two dimensional horizontal variable grid. Monthly Weather Review, 98:810-822.

Berenger, J. P. (1994). A perfectly matched layer for the absorption of electromagnetic-waves. J. Comput. Phys., 114:185-200.

Biot, M. (1952). Propagation of elastic waves in a cylindrical bore containing a fluid. J. Appl. Phys., 23:997.

Browning, G., Kreiss, H. O., and Oliger, J. (1973). Mesh refinement. Mathematics of Computation, 27:29-39.

Cerveny, V. (2001). Seismic Ray Theory. Cambridge University Press.

Chen, K. (1984). Numerical modeling of elasic wave propagation in anisotropic inhomogeneous media: a finite element approach. Ann. Internat. Msg. of soc. Expl. Geophys., Expanded Abstracts, pages 631-632.

Cheng, N. (1994). Borehole Wave Propagation in Isotropic and Anisotropic Media: Three-Dimensional Finite Difference Approach. PhD thesis, Massachusetts Institute of Technology.

Crowder, H. J. and Dalton, C. (1971). Erros in the use of nonuniform mesh systems. Journal of Computational Physics, 7:32-45. 


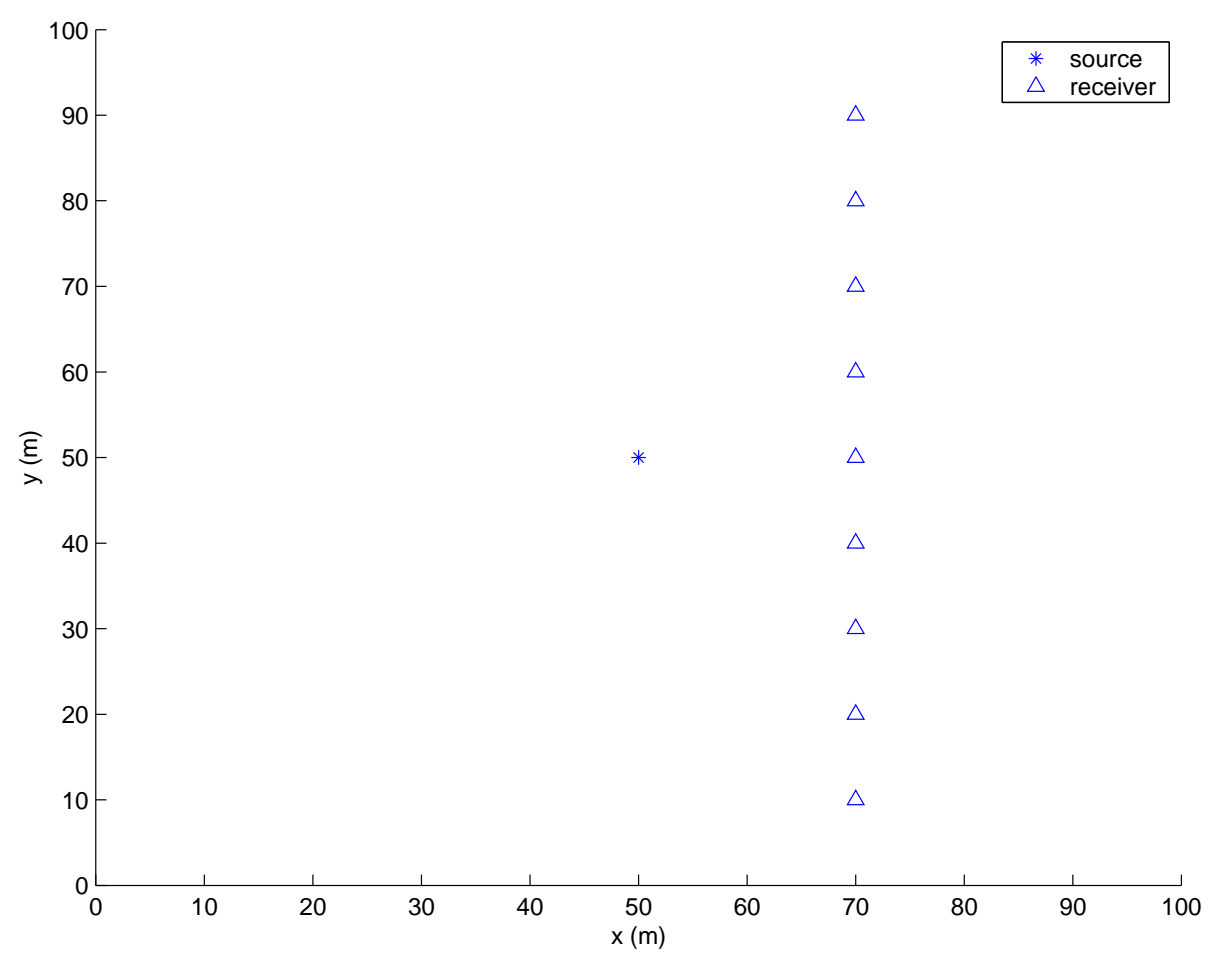

Figure 20: The source and receivers layout for traces in Figure 21. The receivers are lined up in the y direction.

Dablain, M. A. (1986). The application of high-order differencing to the scalar wave equation. Geophysics, $51: 54-66$.

Daubechies, I. (1988). Orthogonal bases of compactly supported wavelets. Commun. Pure and Appl. Math, 41:909-996.

Deslauriers, G. and Dubuc, S. (1989). Symmetric iterative interpolation processes. Constr. Approx., 5:49-68.

Dubuc, S. (1986). Interpolation through an iterative scheme. J. Math. Anal. Appl., 114:185-204.

Emmerich, H. (1992). Psv-wave propagation in a medium with local heterogeneities: a hybrid formulation and its application. Geophysical Journal International, 109:54-64.

Fujii, M. and Hoefer, W. J. (2001). A wavelet formulation of the finite-difference method: Full-vector analysis of optical waveguid junctions. IEEE Journal of Quantum Electronics, 37(8):1015-1029.

Harrington, R. F. (1993). Field computation by moment methods. IEEE Press series on electromagnetic waves. Piscataway, NJ : IEEE Press.

Harrison, E. J. (1973). Three-dimensional numerical simulations of tropicla systems utilizing nested finite grids. Journal of the Atmospheric Sciences, 30:1528-1543.

Hayashi, K. (1999). Variable grid finite-difference modeling including surface topography. Master's thesis, Massachusetts Institute of Technology.

Kalnay de Rivas, E. (1972). On the use of non-uniform grids in finite-fifference equations. Journal of Computational Physics, 10:202-210.

Kelly, K. R., Ward, R. W., Treitel, S., and Alford, R. M. (1976). Synthetic seismograms: A finite-difference approach. Geophysics, 41:2-27. 


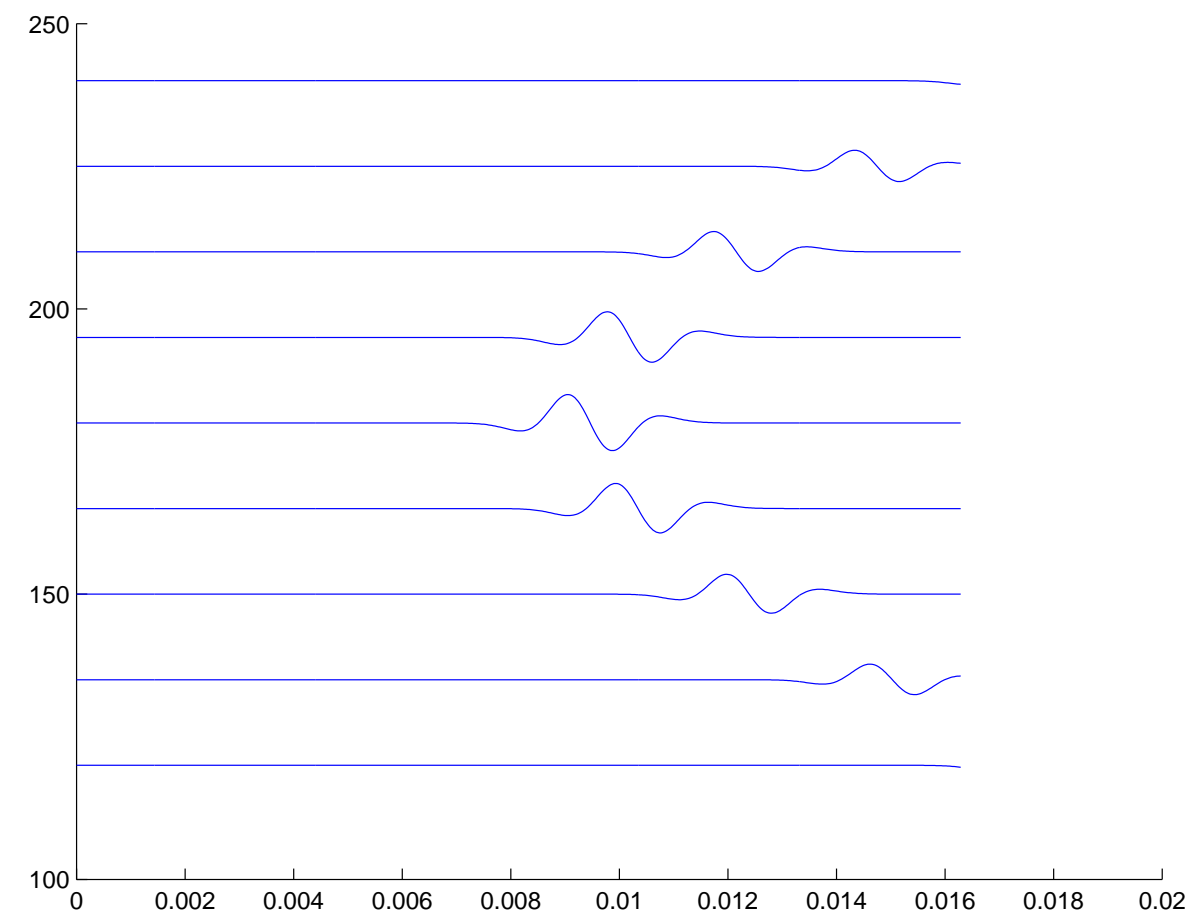

Figure 21: FDTD results (DD4 is used) agree with Green's function results perfectly. The source and receivers setup is shown in Figure 20.

Kosloff, D. and Baysal, E. (1982). Forward Modeling by a Fourier Method. Geophysics, 47:1402-1412.

Krumpholz, M. and Katehi, L. P. B. (1996). Mrtd: New time-domain schemes based on multiresolution analysis. IEEE Transactions on Microwave Theory and Techniques, 44(4):555-571.

Krumpholz, M. and Russer, P. (1993). Two-dimensional fdtd and tlm. Int. J. Num. Modeling, 7(2):141-153.

Krumpholz, M. and Russer, P. (1994). A field theoretical derivation of tlm. IEEE Trans. Microwave Theory Tech., 42(9):1660-1668.

Levander, A. R. (1988). Fourth-order finite-difference p-sv seismograms. Geophysics, 53:1425-1436.

Lilla, A. D. (1997). Finite difference seismic wave propagation using variable grid sizes. Master's thesis, Massachusetts Institute of Technology.

Liu, Q. H. (1999). Perfectly matched layers for elastic waves in cylindrical and spherical coordinates. $J$. Acoust. Soc. Am., 105:2075-2084.

Mallat, S. G. (1997). A Wavelet Tour of Signal Processing. San Diego, CA:Academic.

Muller, G. (1985). The reflectivity method: a tutorial. Geophysics, 58:153-174.

Nolte, B., Rao, R., and Huang, X. (1997). Dispersion analysis of split flexural waves. Borehole Acoustics and Logging/Reservoir Delineation Consortia Annual Report, MIT.

Randall, C. J. (1991). Multipole acoustic waveforms in nonaxisymmetric boreholes and formations. $J$. Acoust. Soc. Am., 90:1620-1631.

Stephen, R. A., Cardo-Casas, F., and Cheng, C. (1985). Finite-difference synthetic acoustic logs. Geophysics, 50:1588-1609. 


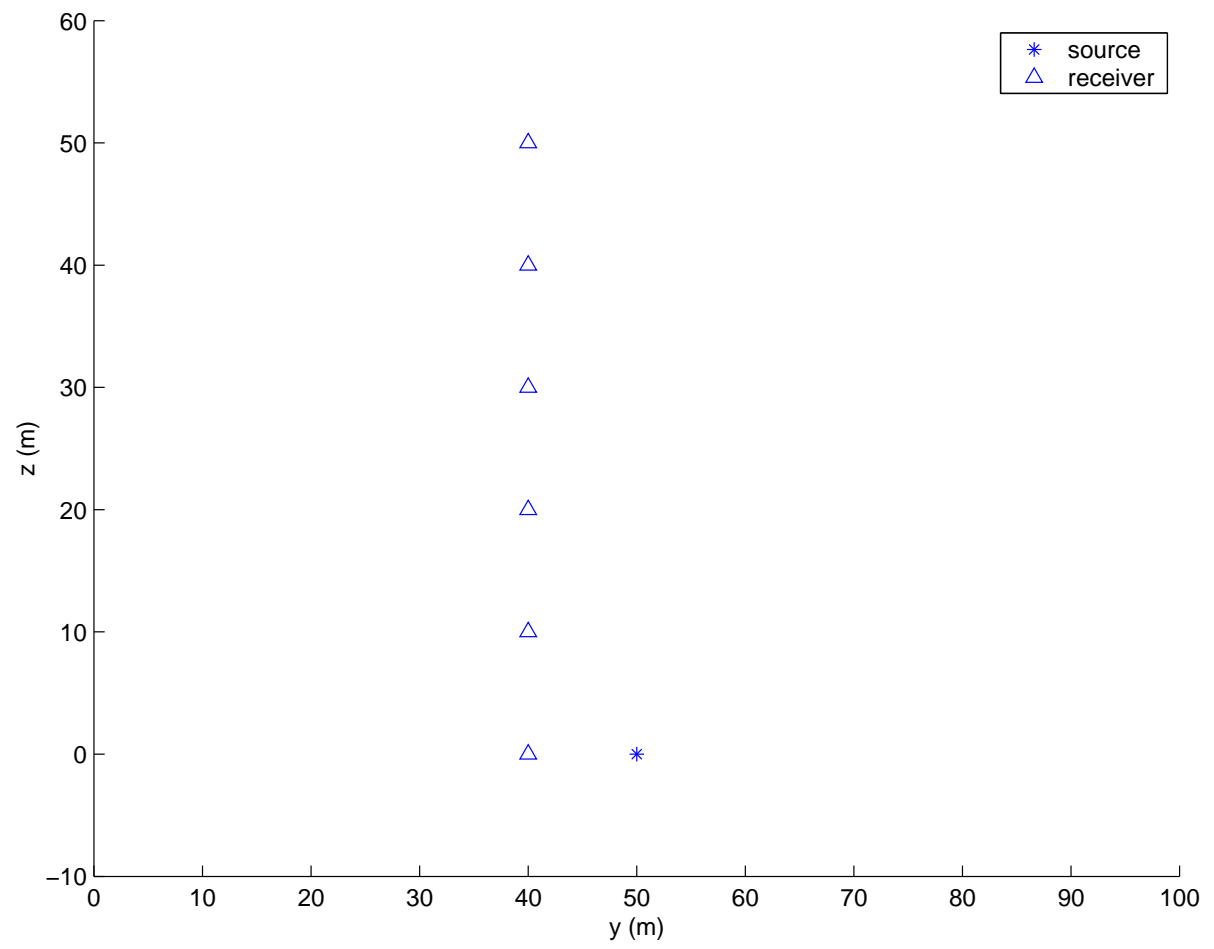

Figure 22: The source and receivers layout for traces in Figure 23. The receivers are lined up in the $\mathrm{z}$ direction.

Strang, G. (1996). Wavelets and Filter Banks. Wellesley-Cambridge Press.

Taflove, A. (1998). Advances in Computational Electrodynamics: The Finite-Difference Time-Domain Method, chapter 1. Artech House.

Virieux, J. (1986). P-sv wave propagation in heterogeneous media: Velocity-stress finite difference method. Geophysics, 51:345-369.

Zheng, Y. and Huang, X. (2001). Anisotropic perfectly matched layers for elastic waves in cartesian and curvilinear coordinate. to be revised for submission. 


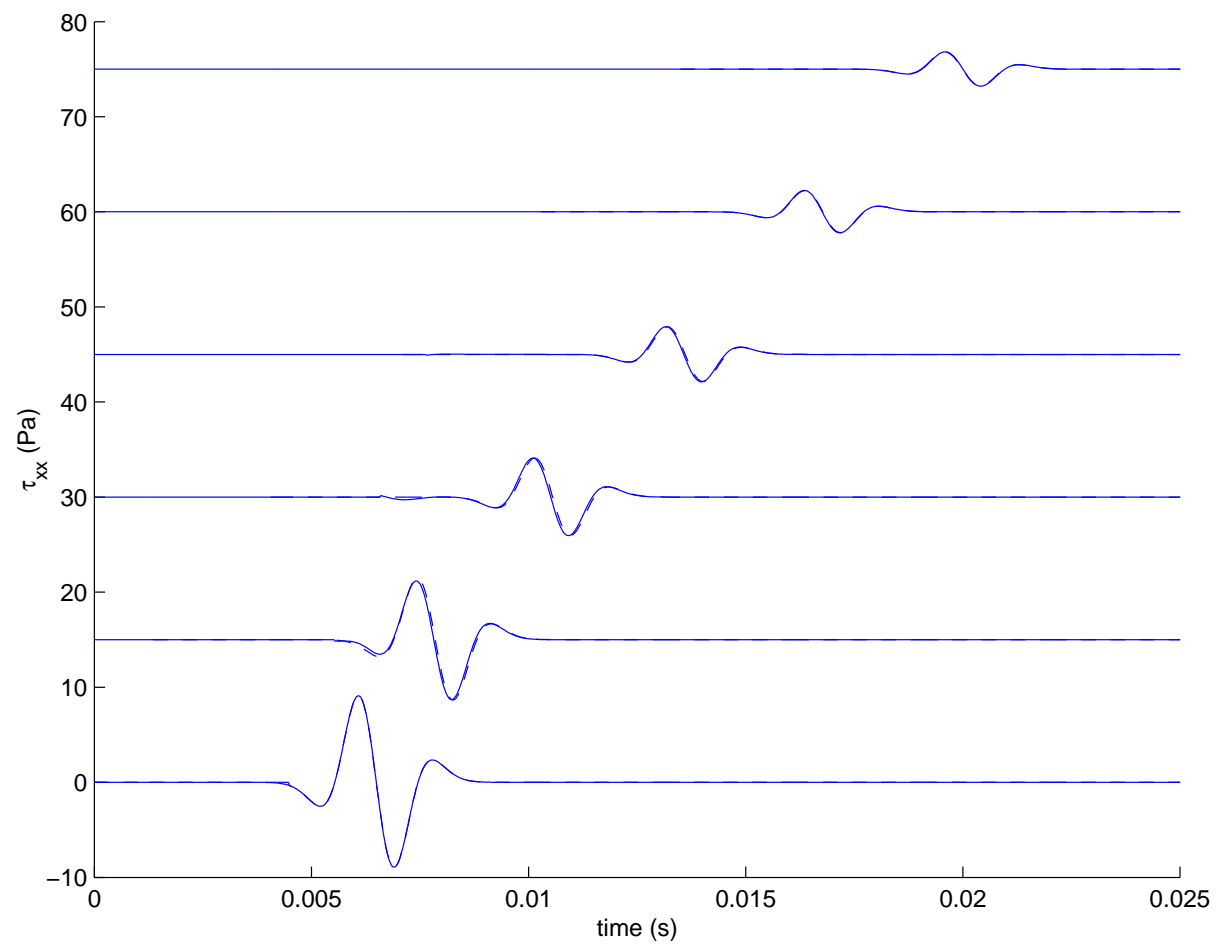

Figure 23: FDTD results (DD4 is used) agree with Green's function results very well. The source and receivers setup is shown in Figure 22.

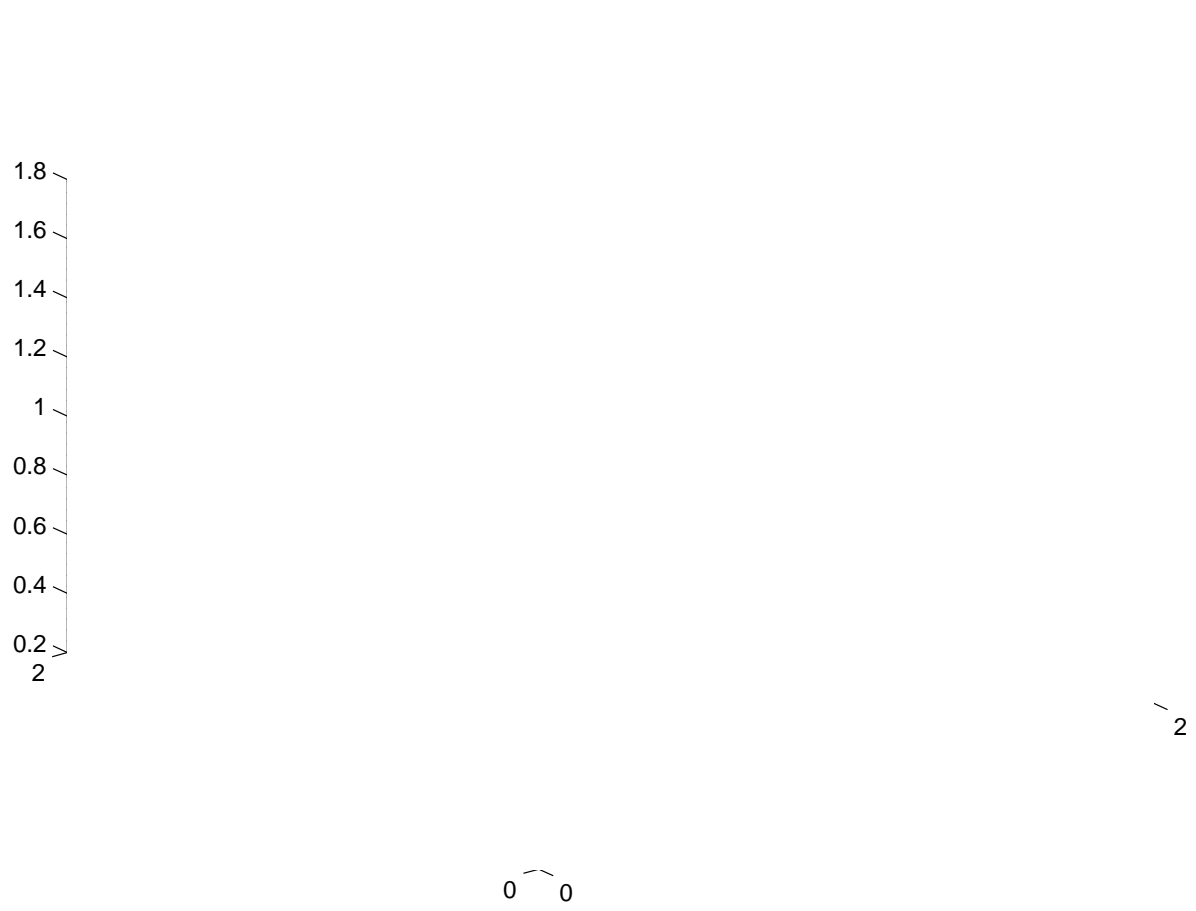


nonuniform grid

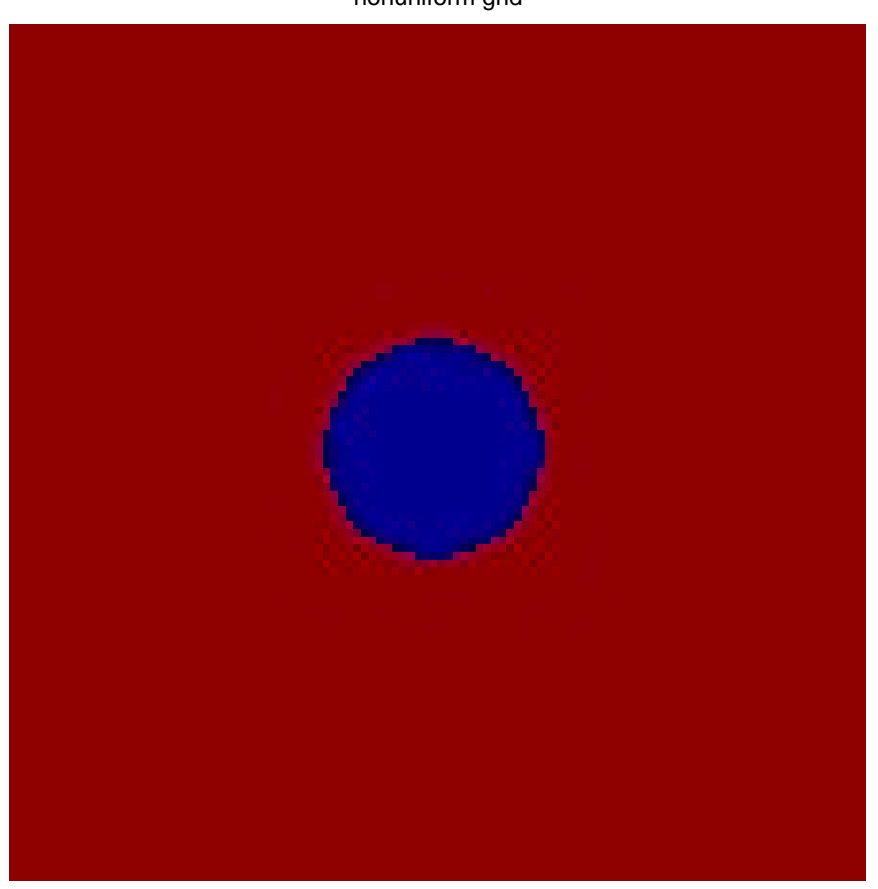

Figure 25: The top view of the discretized borehole using stretching grids shown in Figure 24.

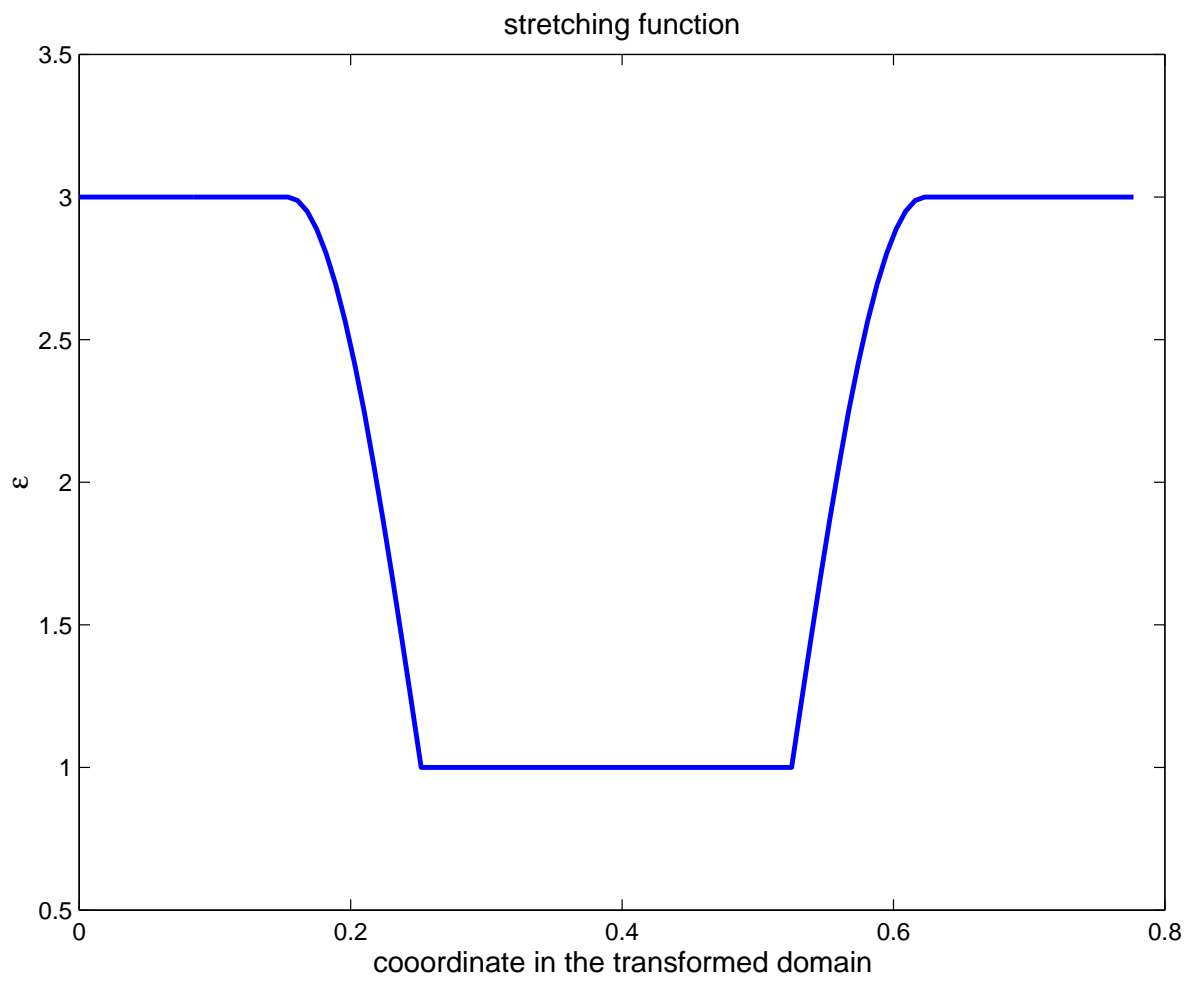

Figure 26: The stretching functions $\epsilon^{1}(\tilde{x})=\epsilon^{2}(\tilde{y})$, used in discretizing the borehole model in Figure 24 . 


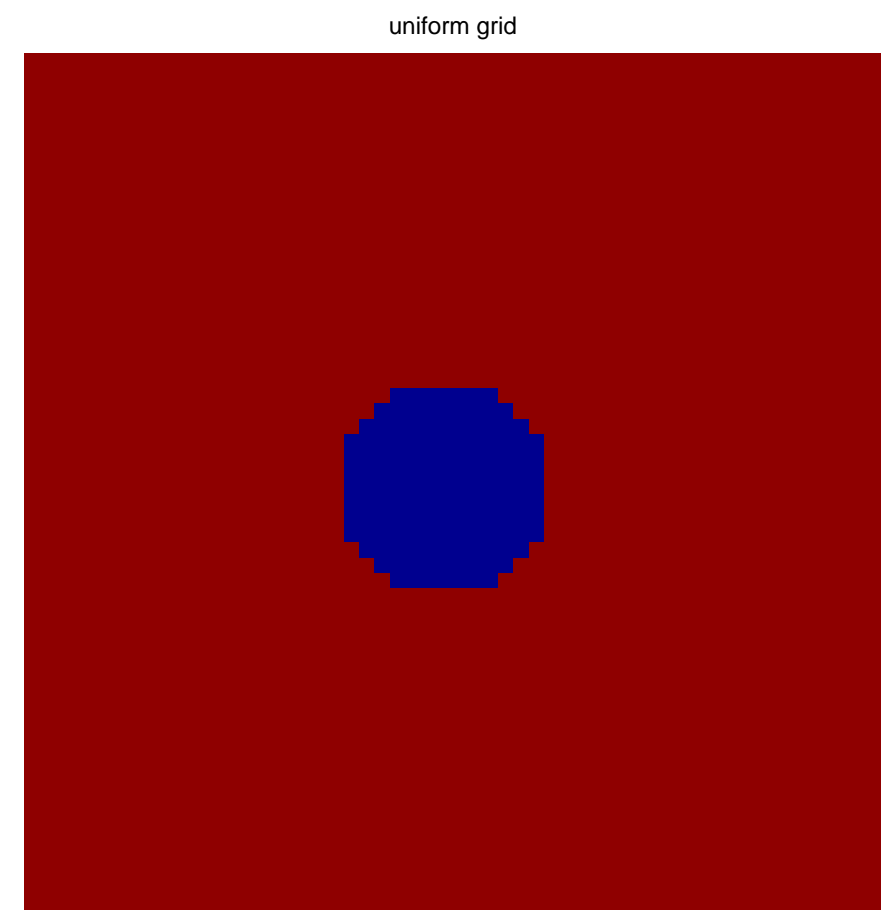

Figure 27: The top view of the discretized borehole using uniform grids with the same grid points used in the stretching grid case (Figure 24). The borehole discretized using the uniform grid mesh is much less smooth and circular than it is in the stretching grid mesh case. 


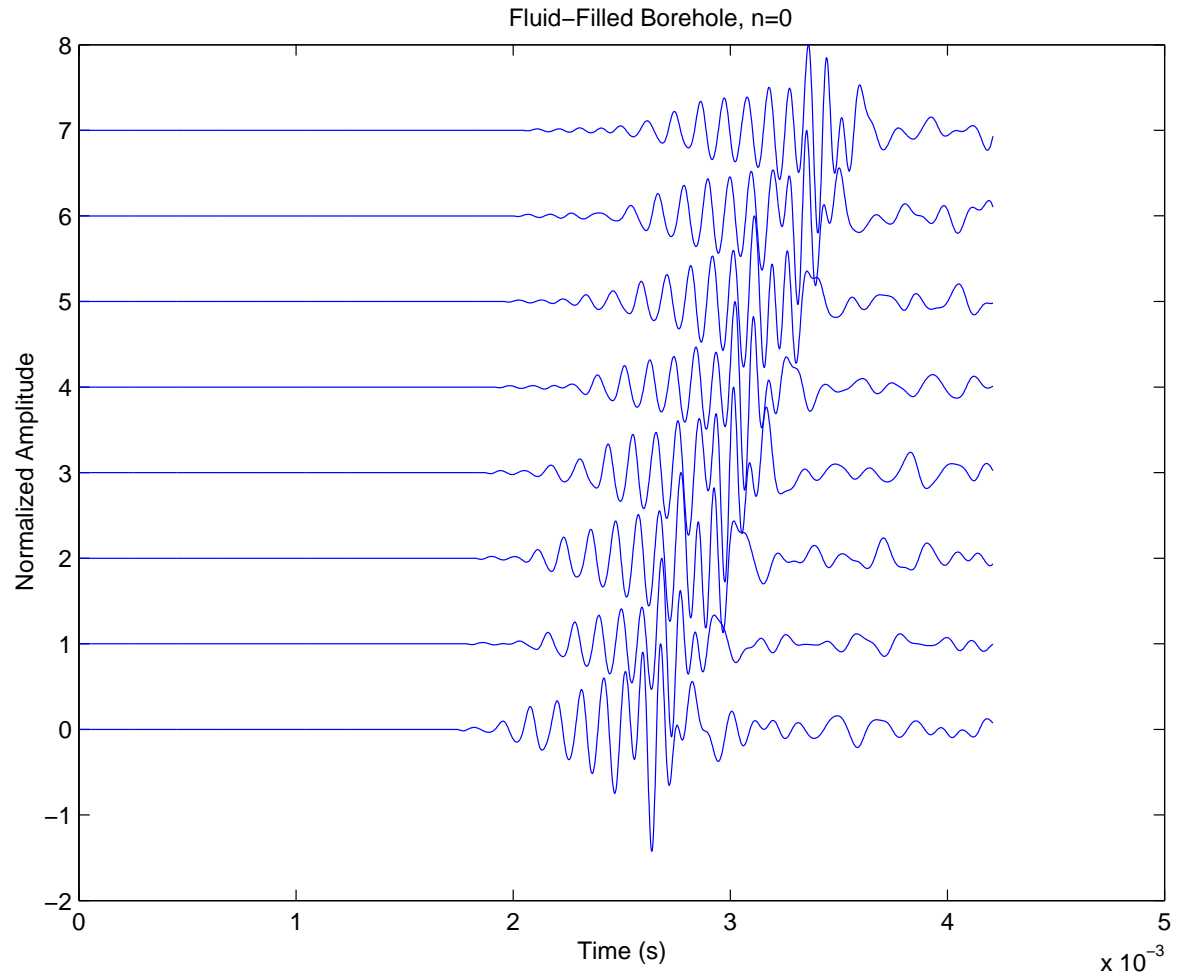

Figure 28: Waveforms from FDTD simulation for the fluid-filled borehole using stretching grids. The spatial differencing scheme is 8 th. The source and receivers are at the $\mathrm{z}$ axis. 


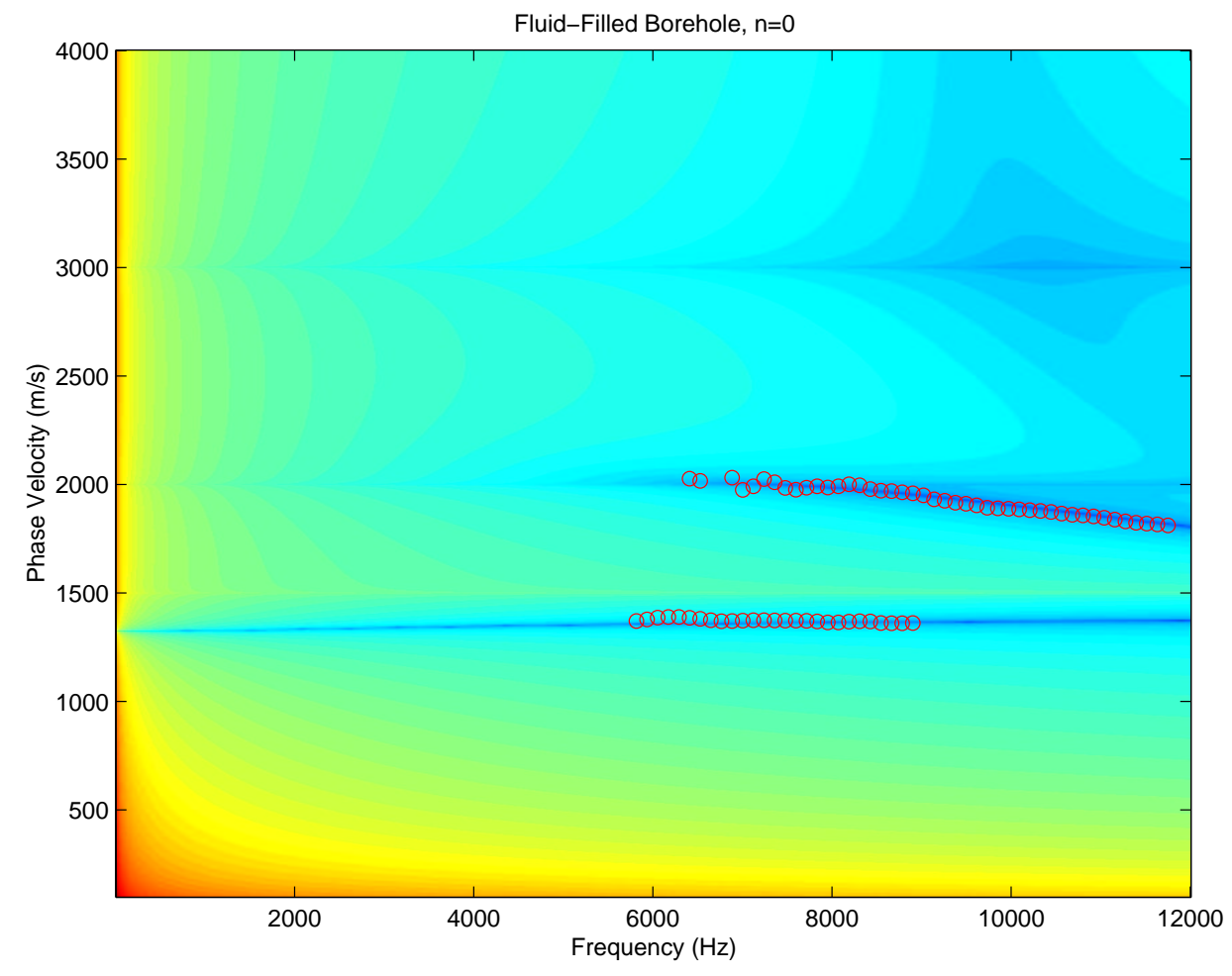

Figure 29: Dispersion curves from analytical solution. The circle lines are from the dispersion analysis of the FDTD results. Two results agree with each other. 


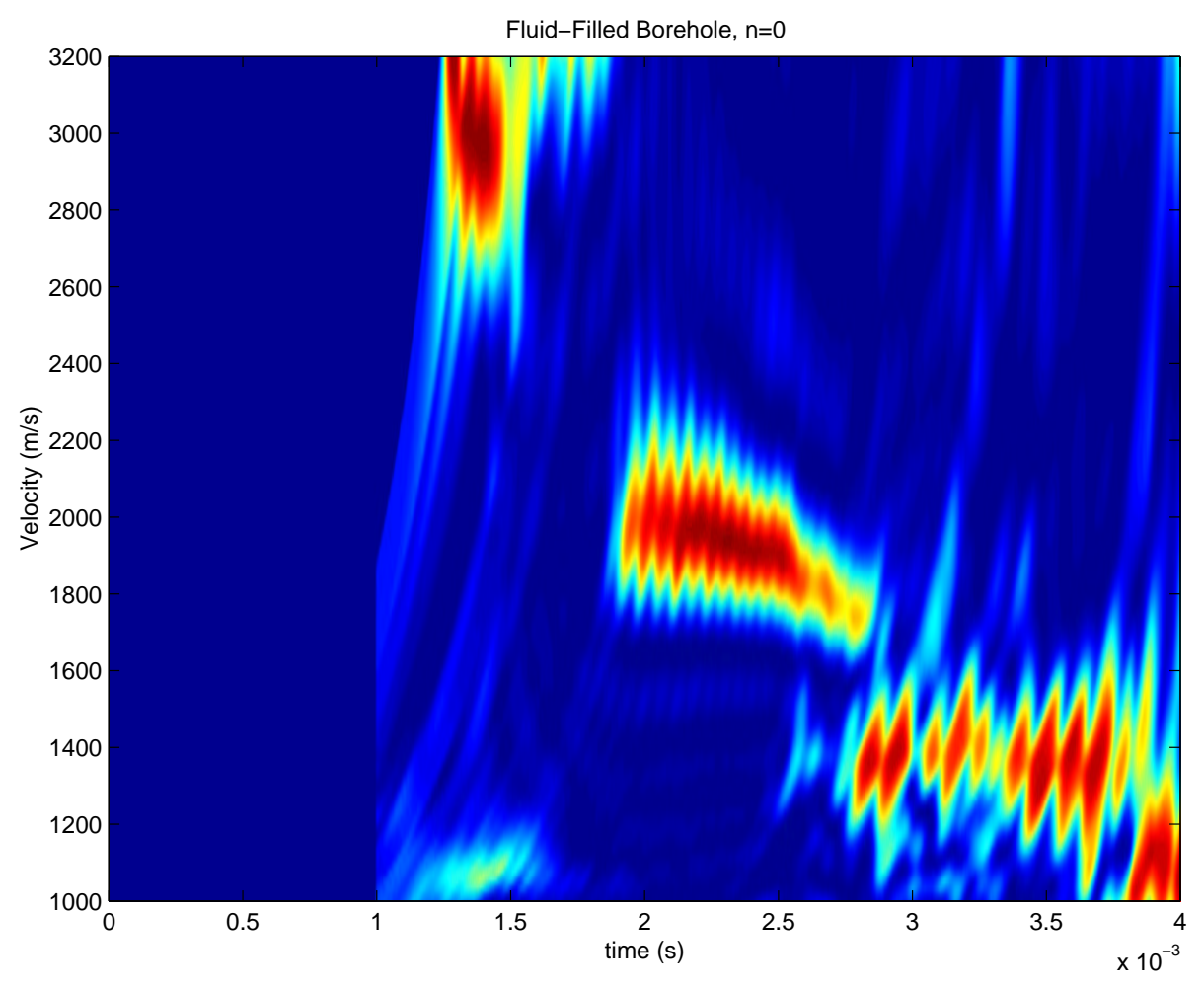

Figure 30: Semblance results of the waveforms obtained from FDTD 28. 


\title{
Simultaneous Inversion for Fast Azimuth and Dispersion of Borehole Flexural Waves Using Cross-Dipole Data
}

\author{
Victoria Briggs, Xiaojun Huang, Rama Rao V.N. and Daniel R. Burns \\ Earth Resources Laboratory \\ Dept. of Earth, Atmospheric, and Planetary Sciences \\ Massachusetts Institute of Technology \\ Cambridge, MA 02139
}

\begin{abstract}
This paper presents an inversion algorithm for obtaining azimuthal angle and borehole flexural wave dispersion in an anisotropic formation. The technique constructs an objective function that can be minimized using standard non-linear inversion methods, which is sensitive to both dispersion and rotation. The method is tested on both synthetic and real borehole data and gives good agreement with traditional processing.
\end{abstract}

\section{Introduction}

Successful petroleum recovery is directly dependent on an accurate reservoir model. With a greater focus in recent years on residual deposits, it becomes increasingly important that reservoir models reflect the true subsurface structure. Imaging techniques such as seismic surveys, give a good representation of the reservoir but the images become meaningless without the proper velocity constraints used for depth conversion. Further, if the medium imaged has a directionally-dependent velocity, the model must be adjusted accordingly.

Formation anisotropy is typically either intrinsic or induced. Hydrocarbon reservoirs in sedimentary basins can consist of large amounts of shale, which is generally intrinsically anisotropic. Anisotropy can also be stress induced by the borehole, by fractures or due to geologic location.

Besides time to depth conversion, anisotropy measurements can be used to calculate 'in-situ' stress fields around the borehole (Huang, 1998) and to determine fracture density and direction (Joyce et al., 1998). Traditionally, anisotropy is estimated from directional borehole measurements which are then corrected before being processed further. Previous work has shown feasability of a joint inversion of the waveforms for formation shear velocities (without dispersion) and azimuth (Tang and Chunduru, 1999) which combines traditional two-step processing into a one-step inversion. In this paper, we describe a method to invert for the full shear wave dispersion curve as well as the azimuth angle.

First, we describe the recorded waveforms in terms of azimuth and formation parameters, and show how the dispersion can be calculated from the array data. Next, we form the objective function that is to be minimized during the inversion and show the results from synthetic data sets. Finally, we invert data from a real tool in a slightly anisotropic clean sand formation.

\section{Cross-Dipole Data}

In order to estimate anisotropy, a directional measurement is essential. Such a measurement can be performed using a cross-dipole acoustic tool. The tool consists of two pairs of acoustic source transducers orthogonally oriented along the $\mathrm{x}$ and $\mathrm{y}$ axes (as shown in Figure 1) and 16 pairs of receivers, with 8 pairs oriented along the $\mathrm{x}$ axis and 8 pairs along the $\mathrm{y}$ axis. This source receiver configuration permits a directional measurement of the formation's acoustic response. Traditional processing involves two steps: first the waveforms are 


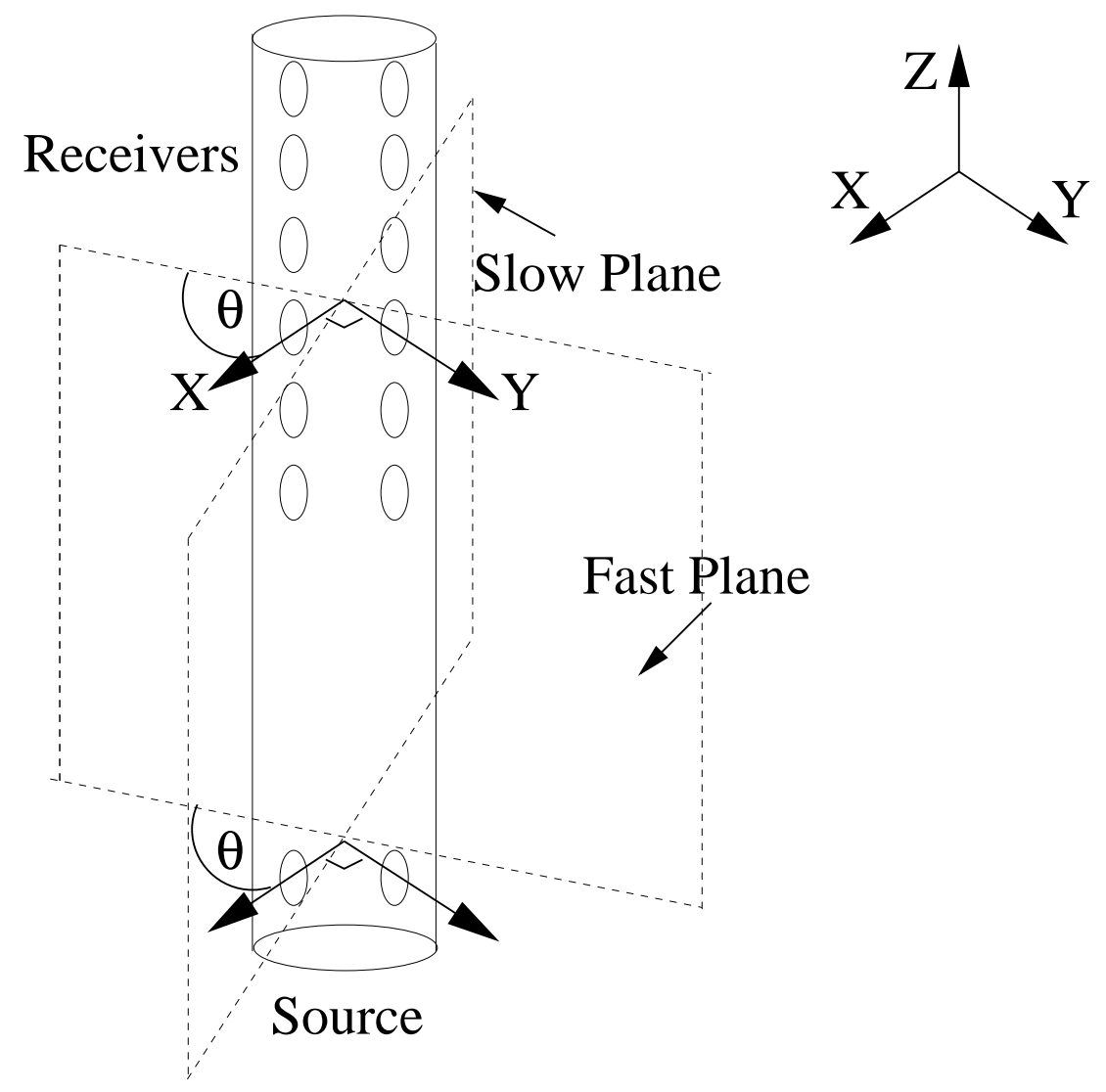

Figure 1: Cross dipole tool. 
rotated to calculate the azimuth and to recompose the data into two principal directions (Alford, 1986); second, the two data sets are used individually to estimate the formation shear properties.

When a dipole source is fired in a borehole a set of antisymmetric borehole-guided modes and the relevant refracted waves (shear/compressional) are excited. The guided modes of lowest cut-off frequency, known as flexural modes, are polarized in the $\mathrm{x}$-y plane, perpendicular to their direction of propagation which is along the z-axis. The flexural mode corresponds to the motion of the borehole flexing from side to side in the formation. When the medium is anisotropic the flexural wave motion splits into a fast and a slow component dependent on the directional velocities of the rock and the frequency component of the excitation.

When the source transducer pair oriented along the $\mathrm{x}$-axis is excited, the displacement vector of the flexural wave generated is also in the $\mathrm{x}$ direction. The fast and slow axes of the formation make an angle $\theta$ with respect to the $\mathrm{x}$ and $\mathrm{y}$ axes so that the displacement vector has projections $\cos \theta$ and $\sin \theta$ on the fast and slow directions, respectively. The fast and slow flexural waves then propagate with their respective (frequency dependent) velocities and are recorded at the receivers. Since the receivers are also oriented along the $\mathrm{x}$ and $\mathrm{y}$ axes, the displacement measured is a second projection of the fast and slow modes back onto the $\mathrm{x}$ and $\mathrm{y}$ axes. For each receiver offset there are two possible source orientations and two possible receiver orientations, from $\mathrm{x}$-source to $\mathrm{x}$-receiver, inline $\mathrm{XX}$, from $\mathrm{x}$-source to $\mathrm{y}$-receiver, crossline $\mathrm{XY}$, and similarly for the $\mathrm{y}$-source, inline $\mathrm{YY}$ and crossline $\mathrm{YX}$.

Let $S_{x}(t)$ and $S_{y}(t)$ represent the source excitation functions for the $\mathrm{x}$ and y oriented dipoles and let $s_{x}(\omega)$ and $s_{y}(\omega)$ be their fourier transforms. Also let $g_{f}^{(n)}(\omega)$ and $g_{s}^{(n)}(\omega)$ be the formation propagation function from the source to the $n^{t h}$ receiver, i.e.,

$$
\begin{aligned}
& g_{f}^{(n)}(\omega)=\exp \left\{i \frac{\omega}{v_{f}(\omega)} z_{n}\right\} \\
& g_{s}^{(n)}(\omega)=\exp \left\{i \frac{\omega}{v_{s}(\omega)} z_{n}\right\}
\end{aligned}
$$

where $v_{f}(\omega)$ and $v_{s}(\omega)$ are the fast and slow frequency dependent phase velocities, respectively, and $z_{n}$ is the distance from the source to the $n t h$ receiver. The signals recorded by the cross dipole tool are therefore given by

$$
\begin{aligned}
x x_{n}(\omega) & =g_{f}^{(n)}(\omega) s_{x}(\omega) \cos ^{2} \theta+g_{s}^{(n)}(\omega) s_{x}(\omega) \sin ^{2} \theta \\
x y_{n}(\omega) & =\left[g_{f}^{(n)}(\omega) s_{x}(\omega)-g_{s}^{(n)}(\omega) s_{x}(\omega)\right] \cos \theta \sin \theta \\
y x_{n}(\omega) & =\left[g_{f}^{(n)}(\omega) s_{y}(\omega)-g_{s}^{(n)}(\omega) s_{y}(\omega)\right] \cos \theta \sin \theta \\
y y_{n}(\omega) & =g_{f}^{(n)}(\omega) s_{y}(\omega) \sin ^{2} \theta+g_{s}^{(n)}(\omega) s_{y}(\omega) \cos ^{2} \theta
\end{aligned}
$$

Assuming the same source function for both the $\mathrm{x}$ and $\mathrm{y}$ transducers, $s_{x}(\omega)=s_{y}(\omega)=s(\omega)$, and letting $f_{n}(\omega)=g_{f}^{(n)} s(\omega)$ and $s_{n}(\omega)=g_{s}^{(n)} s(\omega)$, we can now represent equations 3 to 6 in matrix form

$$
\begin{array}{r}
\left(\begin{array}{ll}
x x_{n}(\omega) & x y_{n}(\omega) \\
y x_{n}(\omega) & y y_{n}(\omega)
\end{array}\right)=\left(\begin{array}{cc}
\cos \theta & -\sin \theta \\
\sin \theta & \cos \theta
\end{array}\right)\left(\begin{array}{cc}
f_{n}(\omega) & 0 \\
0 & s_{n}(\omega)
\end{array}\right) \times \\
\left(\begin{array}{cc}
\cos \theta & \sin \theta \\
-\sin \theta & \cos \theta
\end{array}\right) .
\end{array}
$$

\subsection{Rotation}

We can use equation 7 to create synthetic waveforms for a given source function and dispersion relations. Figures 2 and 3 show the difference between the borehole flexural mode recorded with a tool whose $\mathrm{x}$ and y axes are at an angle $\theta=25^{\circ}$ with the formation fast and slow axes, and the borehole flexural mode recorded with a tool which is aligned with the formation fast and slow axes. In matrix terms, if the tools 

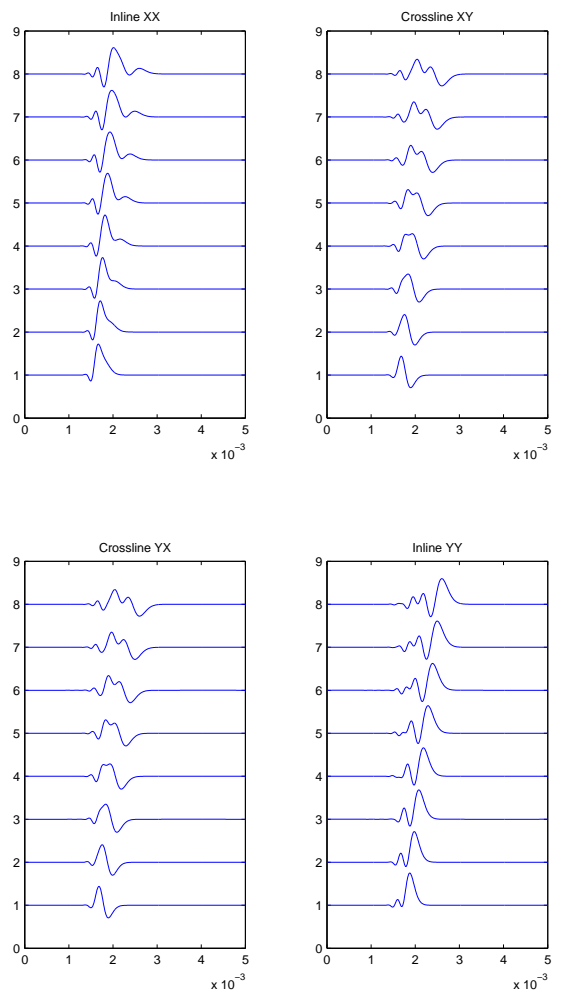

Figure 2: Time series representation of the borehole flexural mode with tool at angle $\theta=25^{\circ}$ with the formation axes.
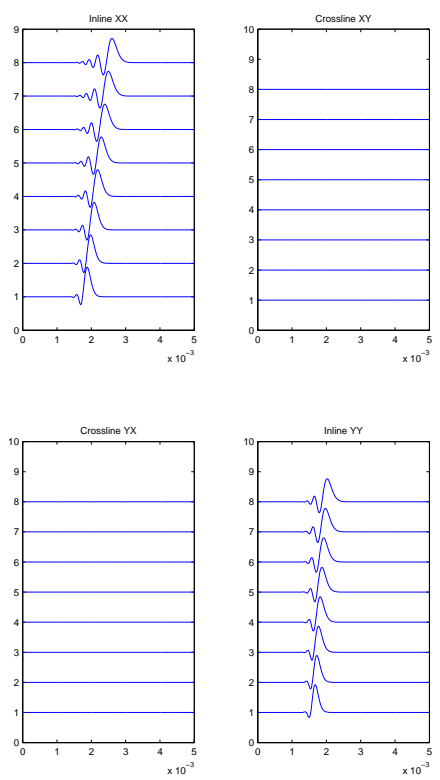

Figure 3: Time series representation of the borehole flexural mode with tool aligned $\left(\theta=90^{\circ}, 0^{\circ}\right)$ with the formation axes . 

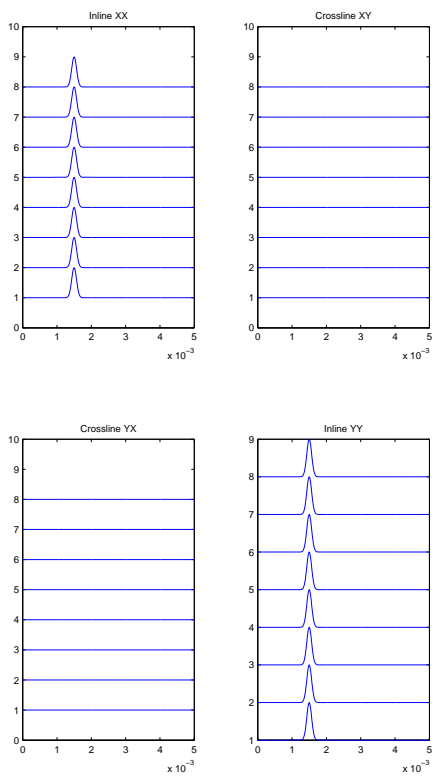

Figure 4: Time series representation of flexural mode after back propagation with the correct dispersion relation .

and formation axes are aligned, the diagonal components of equation 7 are maximized and the off diagonal components vanish.

We can invert 7

$$
\begin{array}{r}
\left(\begin{array}{cc}
f_{n}(\omega) & 0 \\
0 & s_{n}(\omega)
\end{array}\right)=\left(\begin{array}{cc}
\cos \theta & \sin \theta \\
-\sin \theta & \cos \theta
\end{array}\right)\left(\begin{array}{ll}
x x_{n}(\omega) & x y_{n}(\omega) \\
y x_{n}(\omega) & y y_{n}(\omega)
\end{array}\right) \times \\
\left(\begin{array}{cc}
\cos \theta & -\sin \theta \\
\sin \theta & \cos \theta
\end{array}\right)
\end{array}
$$

which gives the fast and slow propagation functions as,

$$
\begin{aligned}
& f_{n}(\omega)=x x_{n}(\omega) \cos ^{2} \theta+\left[x y_{n}(\omega)+y x_{n}(\omega)\right] \sin \theta \cos \theta+Y Y_{n}(\omega) \sin ^{2} \theta \\
& s_{n}(\omega)=x x_{n}(\omega) \sin ^{2} \theta-\left[x y_{n}(\omega)+y x_{n}(\omega)\right] \sin \theta \cos \theta+y y_{n}(\omega) \cos ^{2} \theta .
\end{aligned}
$$

Aditionally it is easy to see from equations 9 and 10 that if $\theta=90^{\circ}$ or $0^{\circ}$ that there will be no $x y_{n}(\omega)$ or $y x_{n}(\omega)$ dependence.

\subsection{Propagation}

Next we undo the effects of propagation through the formation. Figure 4 shows the data from Figure 3 after the propagation has been undone. If the correct dispersion relation is used (i.e., each frequency component is back propagated at the right velocity), only the source function remains at each reciever on the inline arrays and since the correct rotation has been applied, there is no signal on the crossline arrays.

Remembering that $f_{n}(\omega)=g_{f}^{(n)} s(\omega)$ and $s_{n}(\omega)=g_{s}^{(n)} s(\omega)$, we can write equation 8 as,

$$
\begin{aligned}
& \left(\begin{array}{cc}
s(\omega) & 0 \\
0 & s(\omega)
\end{array}\right)=\left(\begin{array}{cc}
e^{-i \frac{\omega}{v_{f}(\omega)} z_{n}} & 0 \\
0 & e^{-i \frac{\omega}{v_{s}(\omega)} z_{n}}
\end{array}\right) \times \\
& \left(\begin{array}{cc}
\cos \theta & \sin \theta \\
-\sin \theta & \cos \theta
\end{array}\right)\left(\begin{array}{ll}
x x_{n}(\omega) & x y_{n}(\omega) \\
y x_{n}(\omega) & y y_{n}(\omega)
\end{array}\right)\left(\begin{array}{cc}
\cos \theta & -\sin \theta \\
\sin \theta & \cos \theta
\end{array}\right) .
\end{aligned}
$$


Which is valid for any receiver $n$.

The propagation function has been moved to the righthand side. We now have a complete expression for the rotation and propagation of a source function to each of the array receivers. In other words, if the correct azimuthal angle and dipserion relations, $v_{f}(\omega)$ and $v_{s}(\omega)$, are used in equation 11, we recover the source function $s(\omega)$ at every receiver.

\section{The Objective Function}

In order to set up the inversion, we need to define an objective function whose minimum is the global best estimate of the parameters we are inverting for. In this case $v_{f}(\omega), v_{s}(\omega)$ and $\theta$. The advantage of combining all the paramters in one objective function is that the inversion searches for the global minimum as opposed to a conditional result. Traditional processing first finds $\theta$, then inverts for velocity. This means that the best estimate for the velocity inversion is constrained by the $\theta$ estimate, thus giving a conditional result.

We know that for the correct azimuth, $\theta$, the back rotated signals $f_{n}(\omega)$ and $s_{n}(\omega)$ will be maximized. We can therfore find the derivative of equations 9 and 10 and set it to zero (or minimize in the case of an inversion) to obtain the azimuthal angle,

$$
\begin{array}{r}
\frac{\partial f_{n}(\omega)}{\partial \theta}=\left(y y_{n}-x x_{n}\right) \sin 2 \theta+\left(x y_{n}+y x_{n}\right) \cos 2 \theta=0 \\
\frac{\partial s_{m}(\omega)}{\partial \theta}=\left(x x_{m}-y y_{m}\right) \sin 2 \theta-\left(x y_{m}+y x_{m}\right) \cos 2 \theta=0
\end{array}
$$

where $n$ amd $m$ refer to the receiver number for the fast and slow arrays, respectively. Since for the correct azimuth, both $\frac{\partial f_{n}}{\partial \theta}(\omega)$ and $\frac{\partial s_{m}}{\partial \theta}(\omega)$ would ideally be zero independently, their difference $\frac{\partial f_{n}}{\partial \theta}(\omega)-\frac{\partial s_{m}}{\partial \theta}(\omega)$ also vanishes. However in order to have an objective function that is sensitive also to correlations in the data i.e., between receivers, we chose to minimize the quantity $\int d \omega\left|\frac{\partial f_{n}}{\partial \theta}(\omega)-\frac{\partial s_{m}}{\partial \theta}(\omega)\right|^{2}$. Note also that when the signal is incorrectly back-rotated, the $f_{n}$ and $s_{n}$ data both contain fast and slow components which are correlated and correctly picked-up by such an objective function

For the propagation, we know that if the correct velocity was used at each frequency, the data at each receiver will be back propagated to the source function. Thus if we undo the propagation correctly and subtract the signal from any receiver pair the absolute value of their difference should vanish.

Therefore our choice of objective funtion is ,

$$
O\left[v_{f}(\omega), v_{s}(\omega), \theta\right]=\int_{\omega} \sum_{n, m}\left|\frac{\partial f_{n}(\omega)}{\partial \theta}-\frac{\partial s_{m}(\omega)}{\partial \theta}\right|^{2}+\left|f_{n}(\omega)-s_{m}(\omega)\right|^{2} .
$$

which is sensitive to both azimuth angle and fast and slow dispersion. Also, this objective function combines all the data from all receivers and it's minimization should therefore enable one to obtain the best fitting parameters (azimuth aswell as fast and slow mode dispersion curves) taking into account the maximum amount of information contained in the data. This is to be contrasted to more traditional methods (first estimate the azimuth independently of dispersion and then seperately analyze the fast and slow mode data) that take only partial account of all the information contained in the data.

Ideally the inversion would search for the best velocity at each frequency as well as the optimal $\theta$. Unfortunately this would lead to a search space with $2 \mathrm{~N}+1$ parameters where $\mathrm{N}$ is the number of samples in frequency. Typically a cross-dipole tool has a source bandwidth of $5 \mathrm{kHz}$ and the number of unknown parameters would be in the order of 1000-10000. Since this is impractical, in terms of inversion, we parametrize the curves $v_{f}$ and $v_{s}$ as a polynomial reducing the inversion space to $2(\mathrm{~N}+1)+1$ dimensions where $\mathrm{N}$ is now the order of polynomial. (Note a first order polynomial has two terms, thus for a straight line fit to the dispersion curve, the inversion has to search for 5 parameters.)

For purposes of this paper we used the Matlab function 'fminsearch.m' to perform the inversion. It uses a Multidimensional unconstrained nonlinear minimization (Nelder-Mead) algorithm. It was used merely for convenience and there is no reason why other non-linear inversion methods should not be sucessful. (least-squares, conjugate gradient or some monte-carlo based method). 
To give some idea of the minimum of the objective function in the inversion space, Figures 5,6 and 7 show results for data generated with $v_{f}(\omega)=4000 \mathrm{~ms}^{-1}$ and $v_{s}(\omega)=2000 \mathrm{~ms}^{-1}$ for all frequencies and $\theta=30^{\circ}$. Using a constant value for the phase velocity as a function of frequency reduces the inversion search space to three, making it easy to visualize.

Using the correct value of $2000 \mathrm{~ms}^{-1}$ for the slow mode, Figure 5 shows the objective function space for the azimuthal angle against the fast mode veloctiy. The minimum can clearly be seen for $v_{f}=4000 \mathrm{~ms}^{-1}$ and $\theta=30^{\circ}$. Figure 6 shows the objective function space for $v_{s}$ and $\theta$ with the correct value for $v_{f}=4000 \mathrm{~ms}^{-1}$. Again the minimum is at the correct values of $2000 \mathrm{~ms}^{-1}$ for $v_{s}$ and $30^{\circ}$ for $\theta$. Similarly Figure 7 shows the minimum in function space for $v_{f}$ and $v_{s}$ with the correct value input for $\theta$.

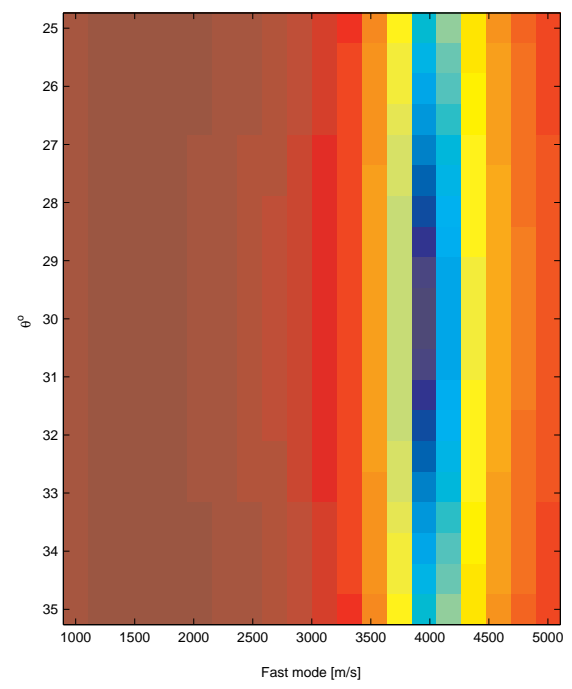

Figure 5: Magnitude of the objective function being minimized: $\theta$ against $v_{f}$ when the correct $v_{s}$ is used. (red is high, blue is low)

\section{Results with Synthetic Data}

In this section we create a synthetic data set to test the inversion. To make the data we simulate a source using a simple Gaussian pulse as a function of time. We transform it to the frequency domain and propagate each frequency component to each receiver using a chosen dispersion relation for both the fast and slow modes. Figure 8 shows the source spectrum overlaid with the dispersion relationships. We have used a second order polynomial for the dispersion curves. It should be noted that the amplitude of the source is not significant. We now have the two principal flexural modes at each inline receiver as a function of frequency. We then use equations 3 to 6 to rotate the principal modes by some angle to get our cross-line components, and finally inverse Fourier transform the signal to get our data as a function of time. The data set used is the same as that in Figure 2, with an azimuthal angle $\theta=25^{\circ}$.

The inversion successfully reproduces the dispersion relationship and finds the correct azimuth, as shown in Figure 9.

\subsection{Synthetic Data with Noise}

Next we added $10 \%$ white noise to the signal in the time domain and the inversion was run again. The inversion now estimates the azimuth to be $22^{\circ}$, which is $3^{\circ}$ less than the true value of $25^{\circ}$. For the low frequencies the inversion matches very well with the true values. At high frequencies the match is not as good, but this is at the tail end of the source frequency spectrum thus there is not much frequency component to constrain the inversion. Figure 11 shows the noisy data after the rotation and back propagation given by 


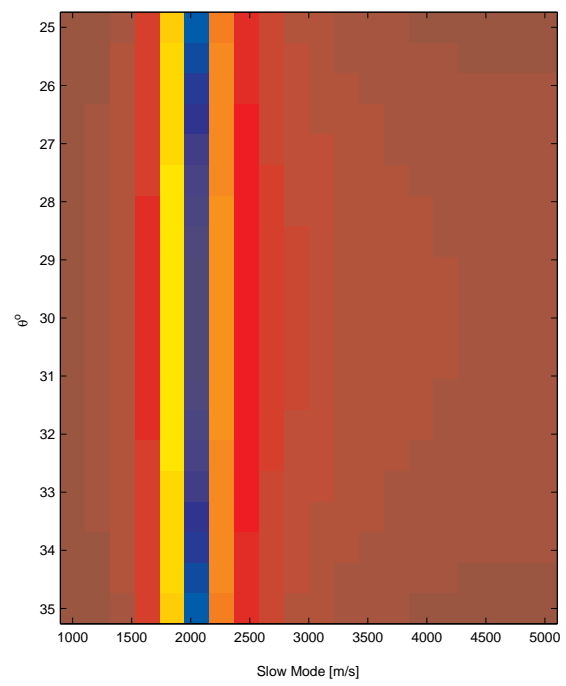

Figure 6: Magnitude of the objective function being minimized: $\theta$ against $v_{s}$ when the correct $v_{f}$ is used.(red is high, blue is low)

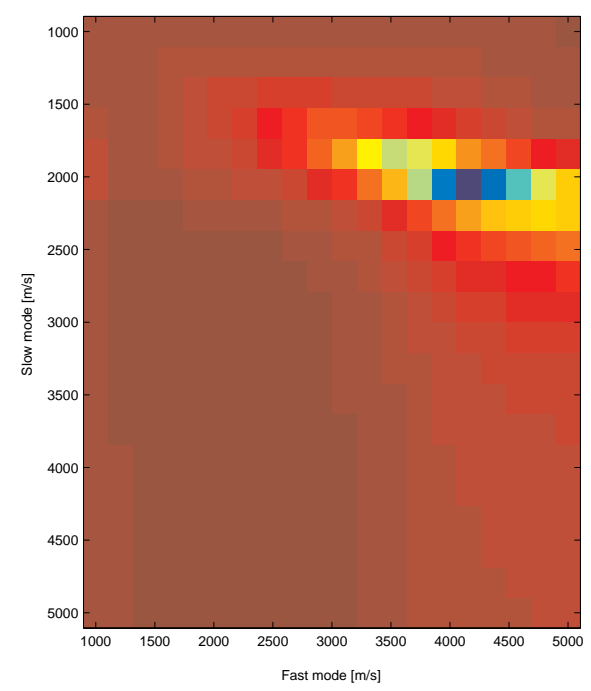

Figure 7: Magnitude of the objective function being minimized: $v_{s}$ against $v_{f}$ when the correct $\theta$ is used. (red is high, blue is low) 


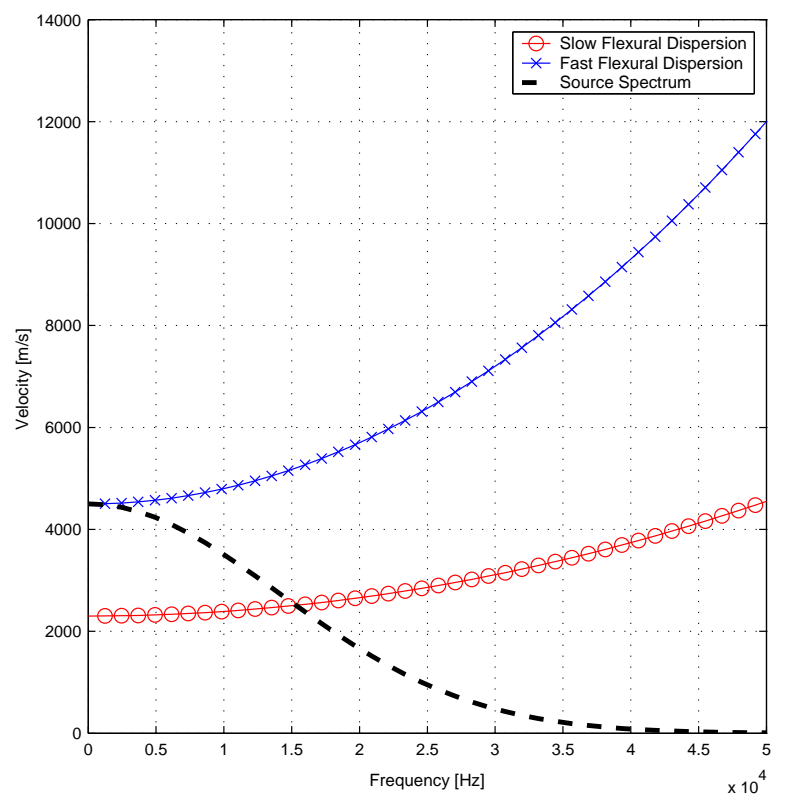

Figure 8: Source spectrum and dispersion relations fpr synthetic data.

the inversion. If the inversion has worked, this figure should look the same as Figure 4, i.e., each inline trace should look like the Gaussian source and the crossline traces should be zero. The effect of the noise remains but otherwise the results are as expected. 

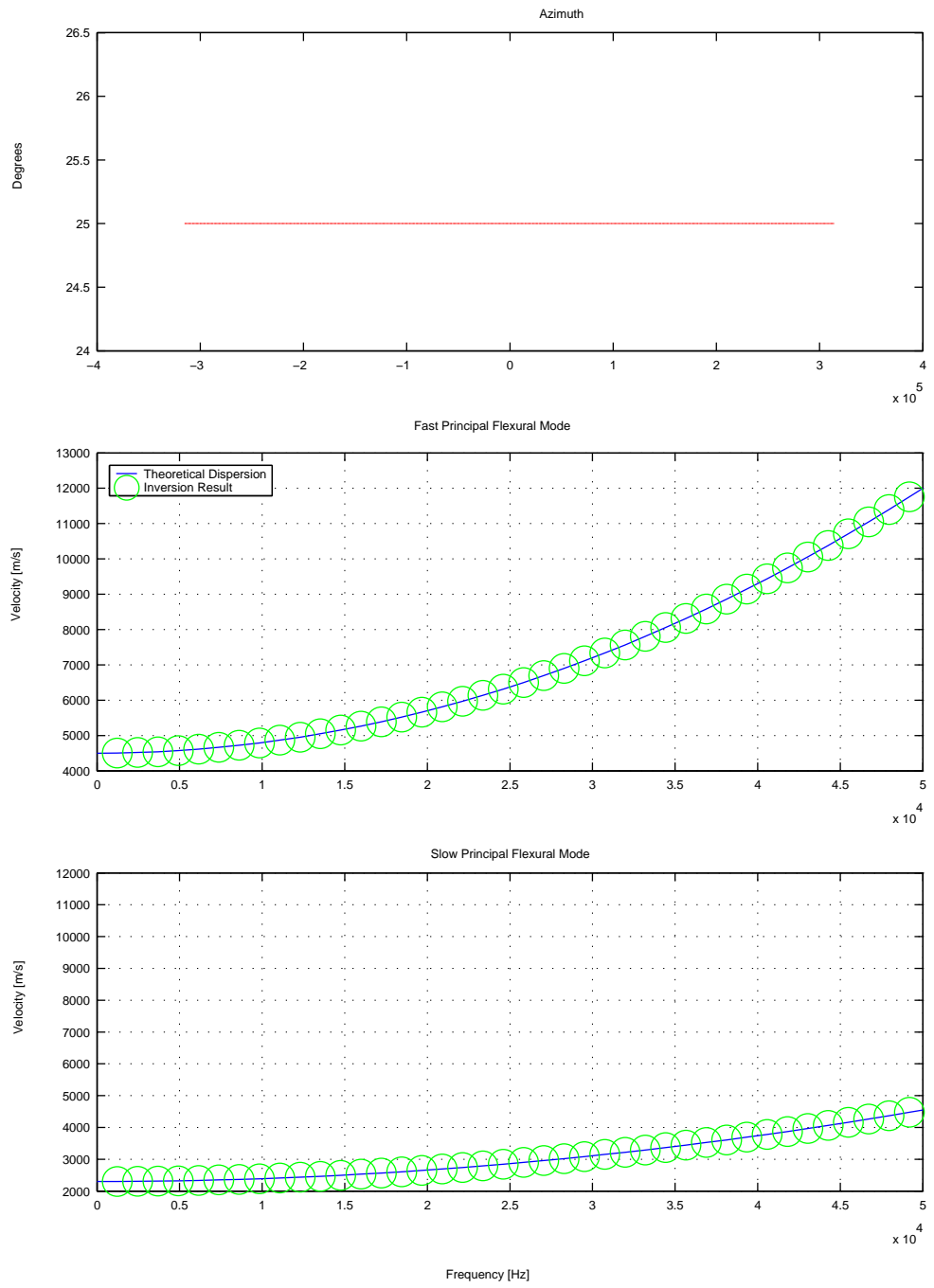

Figure 9: Inversion results. 

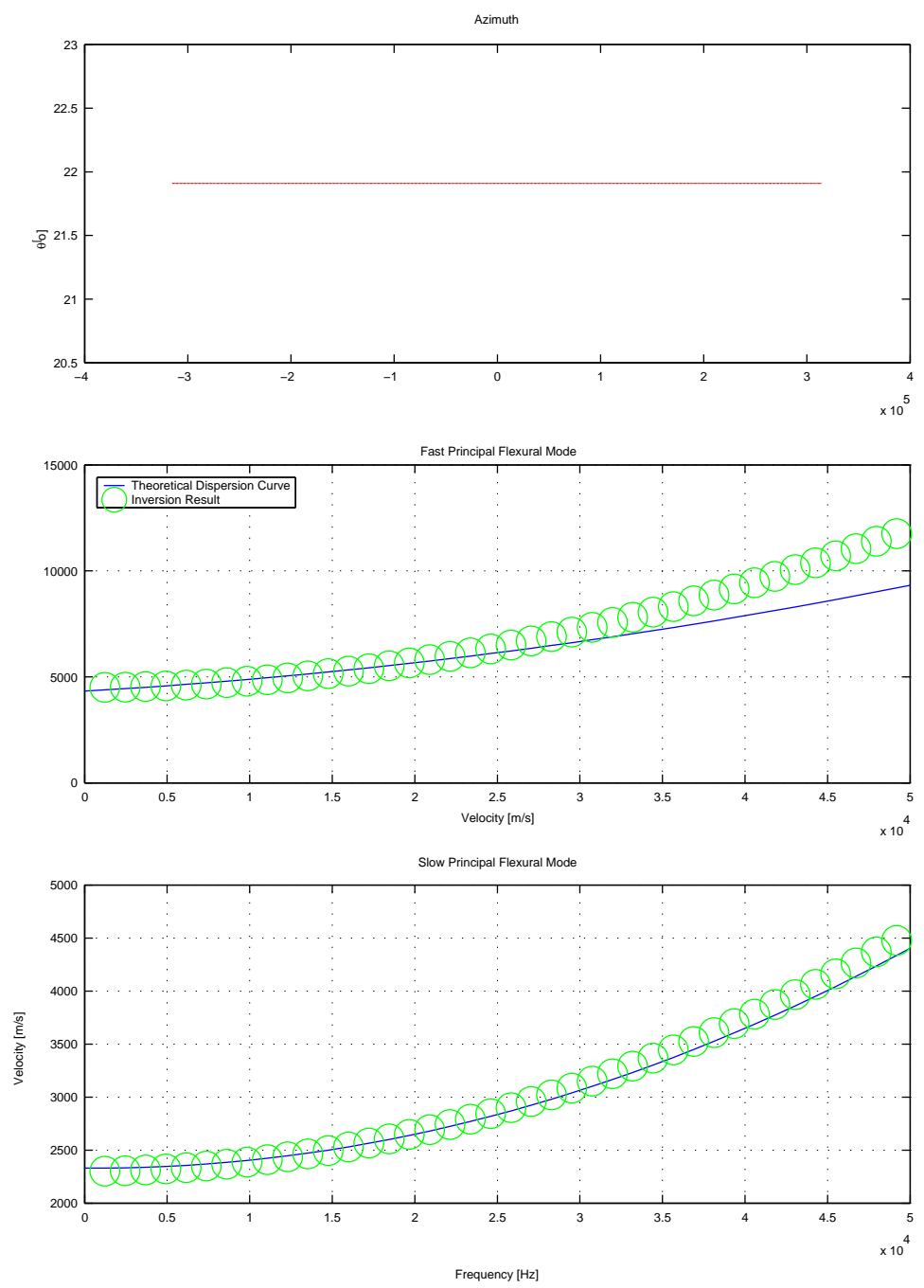

Figure 10: Inversion results for noisy data. 

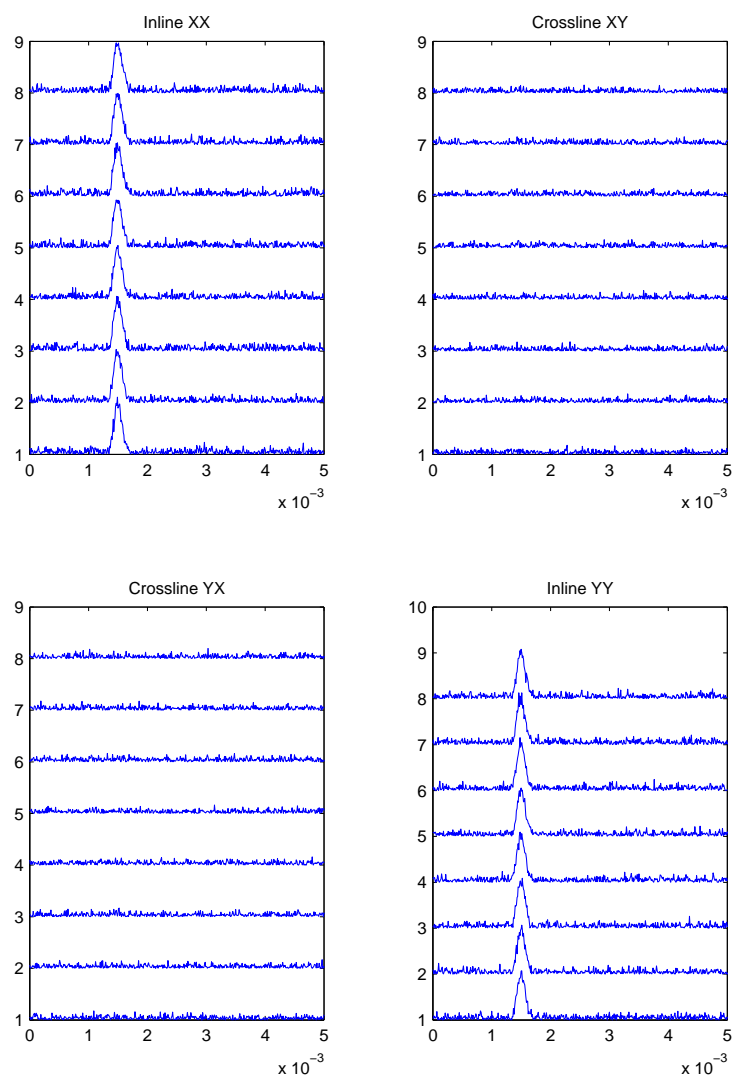

Figure 11: Noisy time series data after being rotated and back propagated with the output from the inversion. 


\section{Inversion of Borehole Data}

In this section we use the inversion algorithm on some real cross-dipole data, Figure 12. The tool used to record the data was Schlumberger's Dipole Shear Sonic Imager (DSI). It contains 8 reciever pairs seperated by $0.1524 \mathrm{~m}$ and two dipole sources with an offset of $3.35 \mathrm{~m}$ from the first reciever pairs. The source has a bandwidth of $80 \mathrm{~Hz}$ to $5 \mathrm{kHz}$. Figure 13 shows the spectrum of the recorded waveforms. The data is from an offshore well in Venezuela and the gamma ray from the same depth suggests a clean sand. Figure 14 shows the waveforms in semblance space. Semblance processing sweeps the data arrays at a fixed time for coherrent energy arriving at a certain velocity, giving an image of phase velocity against time. It can be seen that there are arrivals in the data with phase speeds between 2000 and $3000 \mathrm{~ms}^{-1}$. Schlumberger's processing estimated the formation compressional velocity to be $4419 \mathrm{~ms}^{-1}$ and shear velocity to be $2728 \mathrm{~ms}^{-1}$. Since we have used dipole data for the semblance processing we do not see the compressional arrival.

\subsection{Processing and Results}

Before inverting the waveforms for azimuth and dispersion relations, it is important to isolate the part of the waveform that represents the flexural mode. Since the borehole flexural mode consists if the lowest frequency components and propagates more slowly than the other generated waves, it may be isolated by time windowing and frequency bandpass filtering. Figure 15 shows a short fourier transform of the 1st and last receiver of the XX array. The short fourier transform allows us to identify frequency components arriving at a particular time. The black box on the plots indicates the part of the wave form that was used for the purpose of inversion. The results from the inversion can be seen in Figure 16. The formation exhibits weak anisotropy with the fast and slow flexural modes differing by less than $200 \mathrm{~ms}^{-1}$. Although the borehole flexural mode is highly dispersive in the right frequency interval, it does not change significantly over the frequencies sampled in the data. The inversion results agree very well with the semblance processing of the data, both flexural mode phase speeds fall in the coherrent region on the semblance plot. In addition the inversion results show slight anisotropy which is not apparent from the semblance alone. 

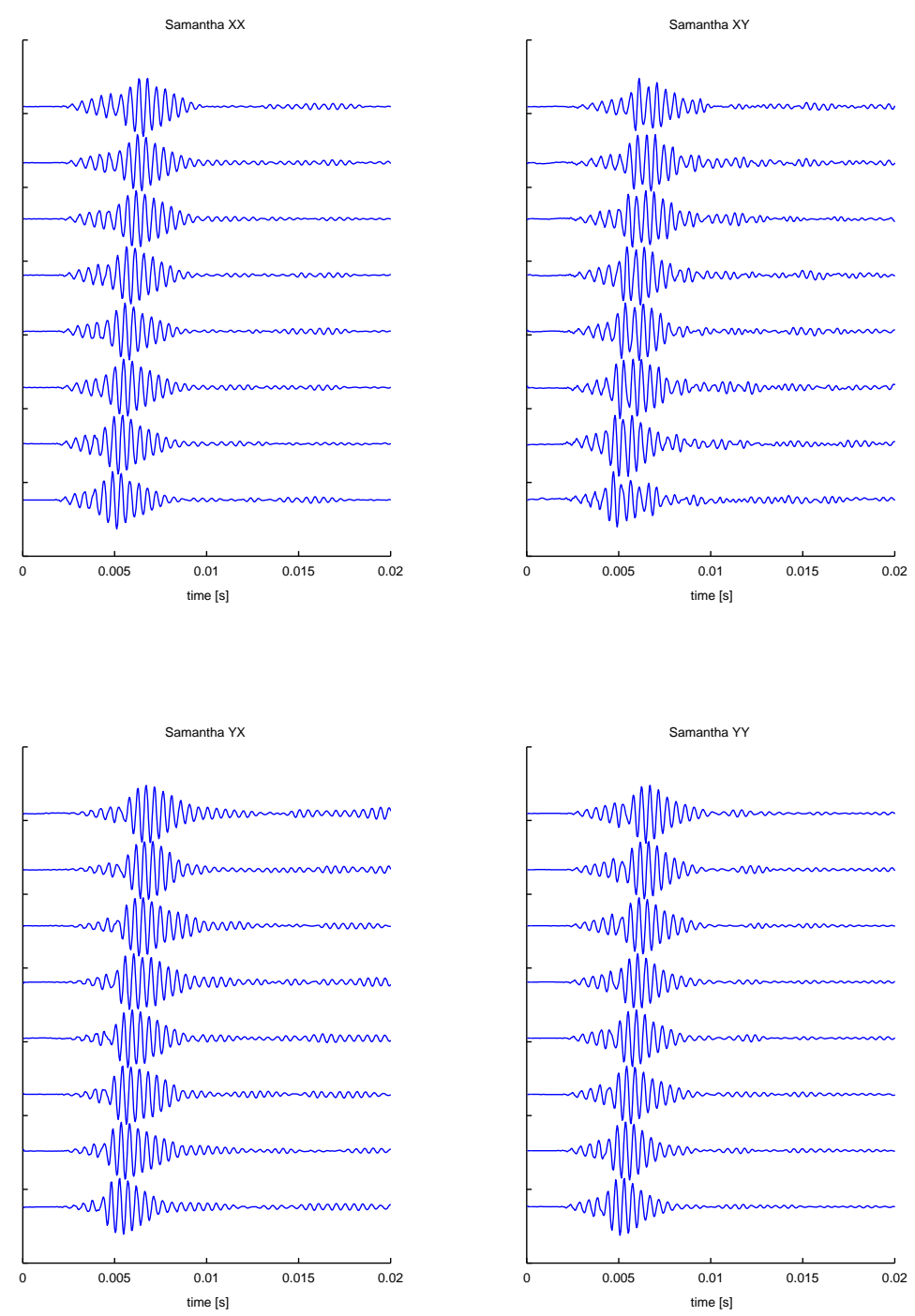

Figure 12: Cross dipole Data recorded by DSI tool in an offshore well in Venezuela. 


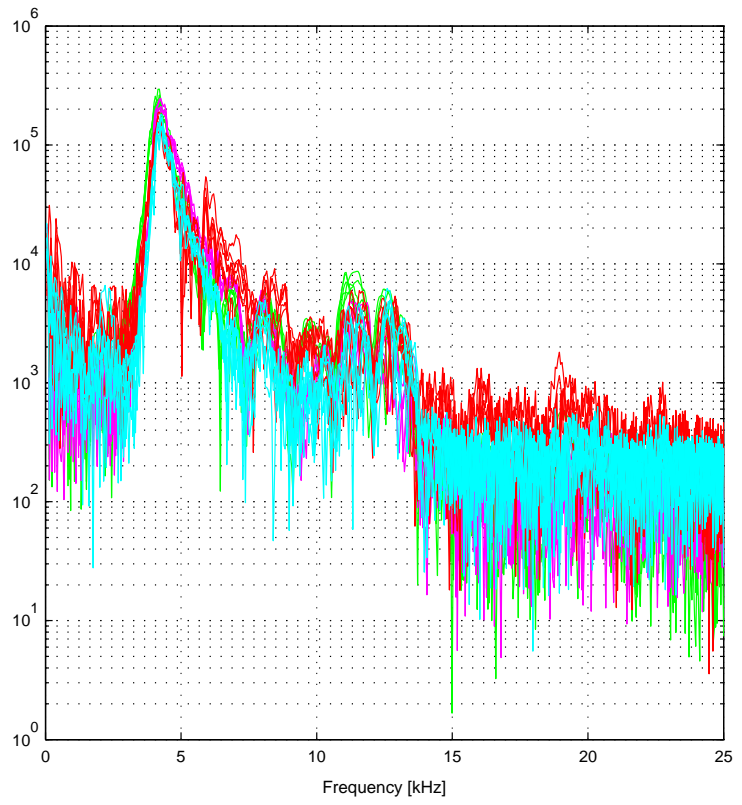

Figure 13: Frequency spectrum of Venezuela data.

\section{Conclusions and Further Work}

We have presented a new method for inverting borehole flexural waveforms, in anisotropic formations, for azimuth and frequency dependent phase velocities. The inversion allows a traditional multi-step processing to be condensed into a one-step inversion thus allowing a global best solution to be found.

The algorithm was shown to successfully invert synthetic data for the exact starting parameters used to form the data set. Results for noisy data also gave very good agreement. The process was also applied to field data and agreed well with traditonal processing methods. The results show a weak anisotropy that was missed by processing the waveforms individually. Further work needs to be done to optimize the starting guess for the inversion procedure as the computation time is directly correlated to the 'goodness' of the starting guess. In this paper we used a polynomial parametrization for the fast and slow dispersion curves, further work can be done to find better representations as this too directly influences the computation time. It would also be interesting to examine the possibility of a multi-mode inversion as this would remove the need to filter the flexural mode from the waveforms which is not always a trivial step.

\section{Acknowledgments}

This work was supported by the Borehole Acoustics and Logging Consortium at the Massachusetts Institute of Technology, and by Department of Energy Grant \#DE-FC26-02NT15346. 

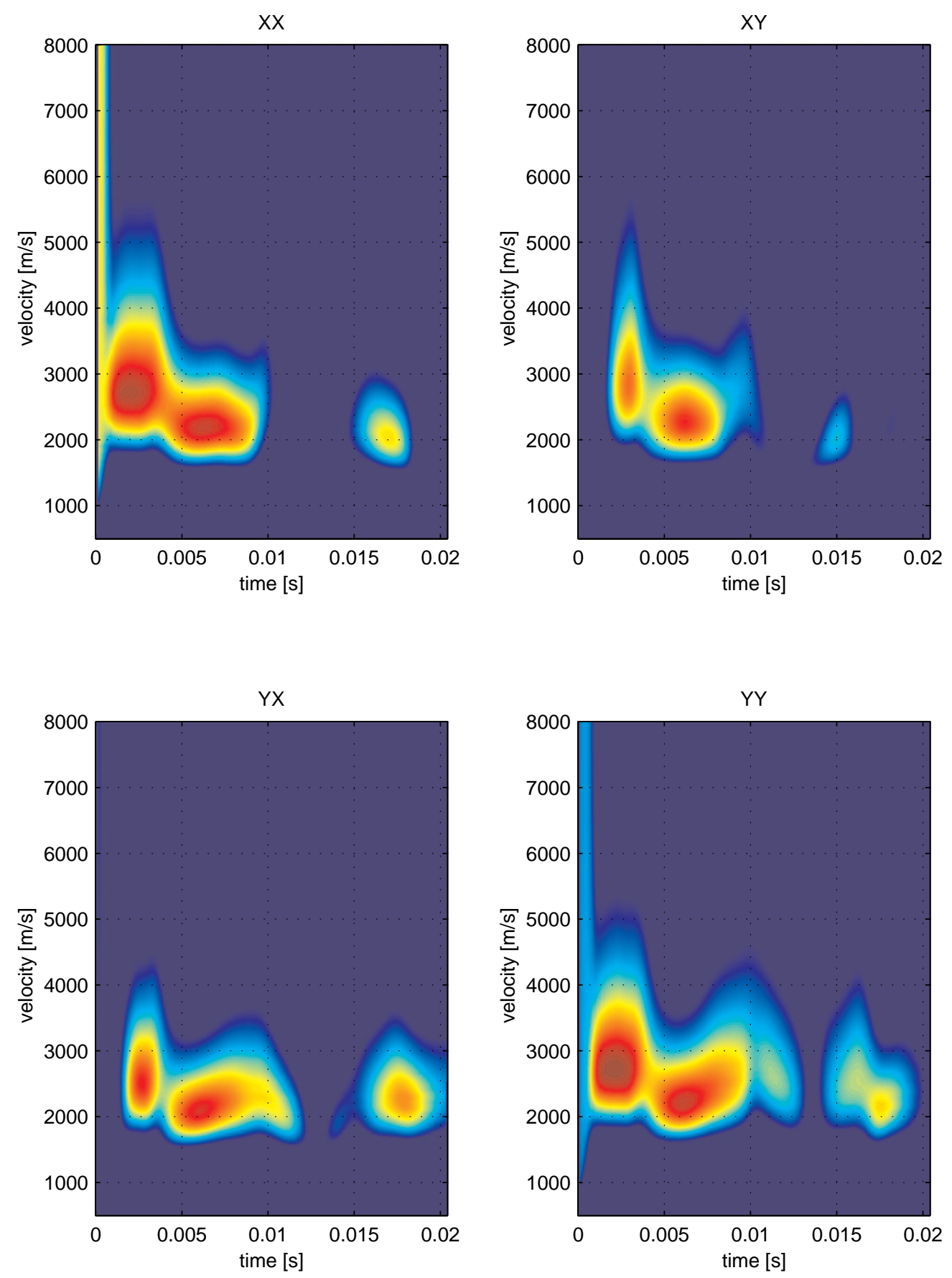

Figure 14: Semblance processing results of Venezuela data. 

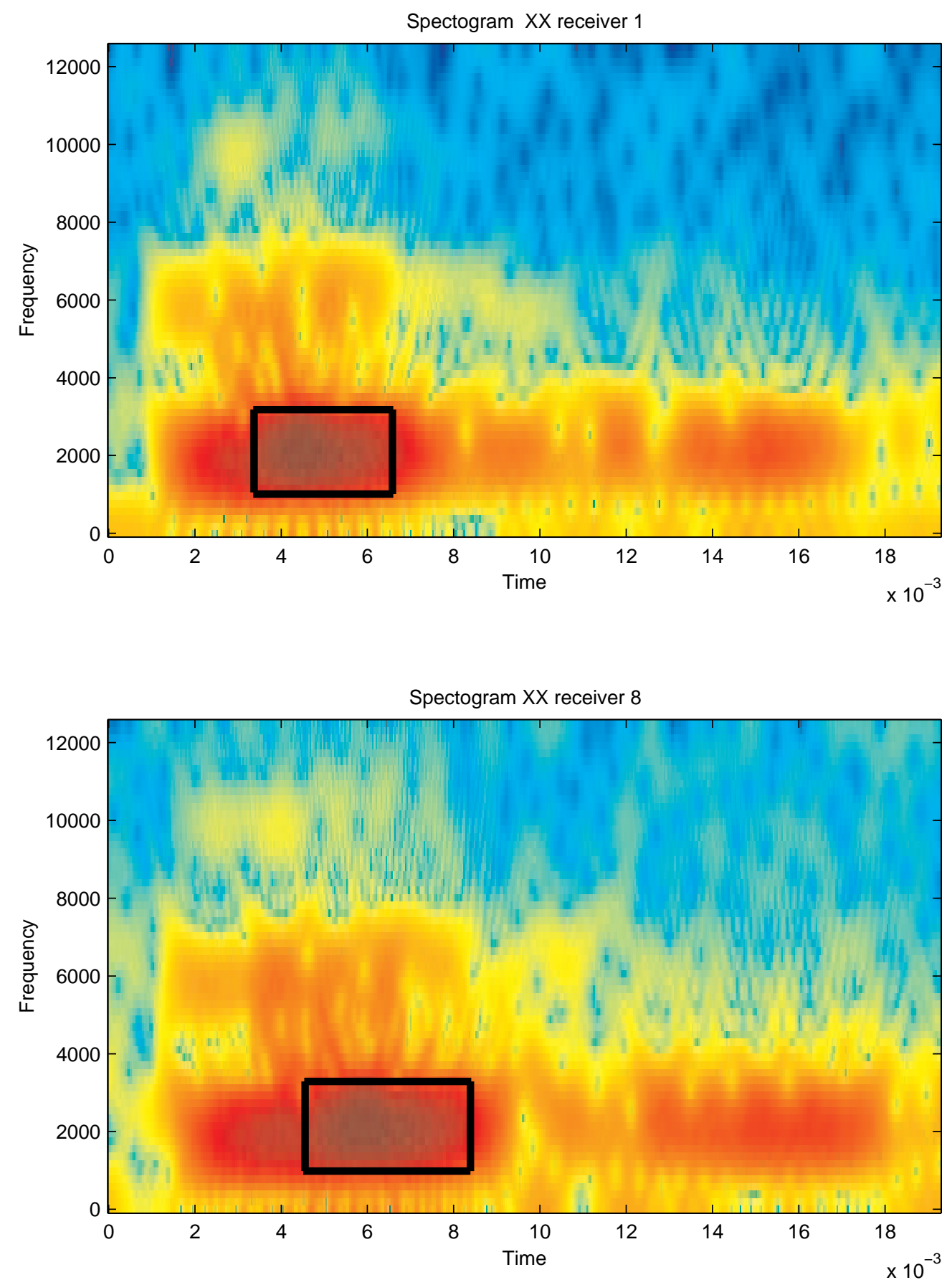

Figure 15: Spectogram of the 1st and last waveforms from the XX array (Venezuela data). 
Azimuth
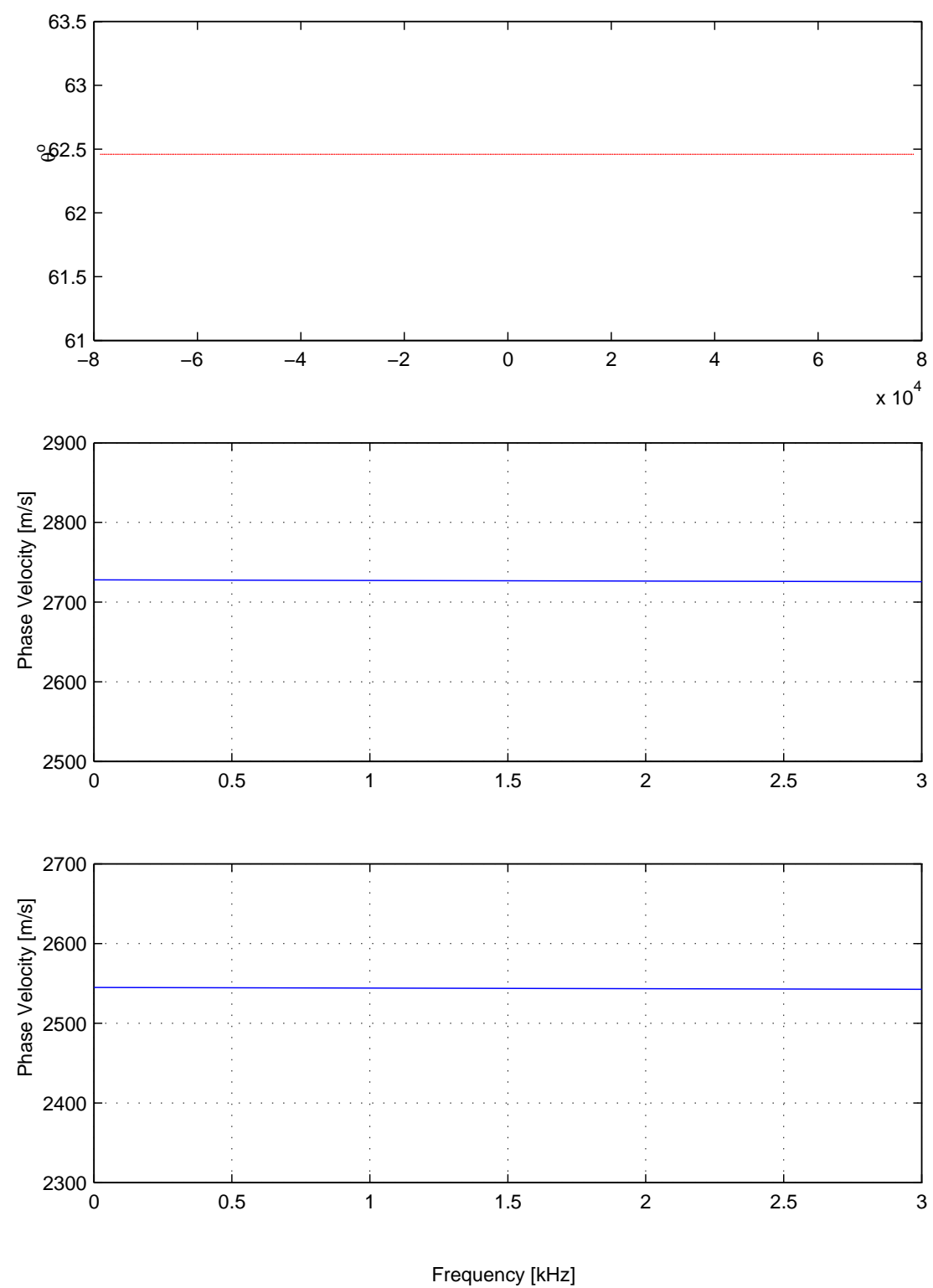

Figure 16: Inversion results when using a 2nd order polynomial to fit dispersion curves (Venezuela data). 


\section{References}

Alford, R.M., 1986, Shear data in the presence of azimuthal anisotropy, 56th Ann. Int. Mtg., Soc. Expl. Geophys., Expanded Abstracts 476-479.

Huang, X., 1999, A study of tectonic stresses in the Earth using standard acoustic logging, Generals Exam Paper, E.A.P.S. M.I.T.

Joyce, R. and Patterson, D., 1998 Advanced interprestation of fractured carbonate reservoirs using fourcomponent cross-dipole analysis, 39th Ann. Mtg., Soc. Prof. Well Log Anal., Paper R.

Tang, X. and Chunduru, R., 1999, Simultaneous inversion of formation shear-wave anisotropy parameters from cross-dipole acoustic-array waveform data, Geophysics, 64 1502-1511. 


\title{
Fluid Flow Simulation in Fractured Reservoirs
}

\author{
Sudipta Sarkar, M. Nafi Toksöz, and Daniel R. Burns \\ Earth Resources Laboratory \\ Department of Earth, Atmospheric, and Planetary Sciences \\ Massachusetts Institute of Technology \\ Cambridge, MA 02139
}

\begin{abstract}
The purpose of this study is to analyze fluid flow in fractured reservoirs. In most petroleum reservoirs, particularly carbonate reservoirs and some tight sands, natural fractures play a critical role in controlling fluid flow and hence production. Uncertainties involved in the understanding of fracture architecture and properties often propagate in the construction of reservoir flow models. The state-of-the-art reservoir simulation packages used widely in the oil industry often do not take into account the complex random geometry of real fracture systems that can vary from one grid-block to another, and sometimes even within a single grid-block. The reason for this is two-fold: there exists no technology as yet to image the micro-fractures in-situ, and most of the reservoir modeling software does not use micro-scale flow equations to model the change of flow variables. Fractures are highly conductive channels for flow among all types of porous-permeable media, Flow through them can vary widely depending on different fracture properties, such as fracture apertures, densities, asperities, etc.

The objective of this study is to understand fluid flow in fractures using a finite difference approach, and to analyze the effects of fracture properties on flow mainly through visualization. Contrary to the conventional macro-scale modeling approach, micro-scale simulation is carried out. The ultimate goal is to incorporate this information into reservoir scale modeling schemes.
\end{abstract}

\section{$1 \quad$ Introduction}

The flow of fluids through fractures in rocks is a process that has importance for many areas of the geosciences, ranging from ground-water hydrology to oil. Research on fluid flow in fractures and in fractured porous media has a history that spans nearly four decades. This research has focused on four principal aspects of fracture flow: 1) development of conceptual models, 2) development of analytical and numerical solution schemes, 3 ) description of fracture hydraulic characteristics in static and deforming media, and 4) development of stochastic techniques to describe fracture flow and hydro-geologic parameter distributions. Bear et al. (1993) reviewed the research on fracture flow phenomena extensively. The thrust of our study is to develop a numerical model based on the basic physics of fluid flow in the earth, and to implement it to simulate fracture flow and analyze different flow properties under varying fracture characteristics.

Several conceptual models have been developed for describing fluid flow in fractured porous media. Fundamentally, each method can be distinguished on the basis of the storage and flow capabilities of the porous medium and the fracture. The storage characteristics are associated with porosity, and the flow characteristics are associated with permeability. Four conceptual models have dominated the research: 1) explicit discrete fracture, 2) dual continuum, 3) discrete fracture network, and 4) single equivalent continuum. In addition, multiple-interacting continua and multi-porosity/multi-permeability conceptual models (Sahimi, 1995) have recently been introduced in the literature. Further distinctions can be drawn on the basis of the spatial and temporal scales of integration, or averaging, of the flow regime. Bear and Berkowitz (1987) describe four scales of concern in fracture flow: 1) the very near field, where flow occurs in a single fracture and porous medium exchange is possible; 2) the near field, where flow occurs in a fractured porous medium and each fracture is described in detail; 3) the far field, where flow occurs in two overlapping continua with mass exchanged through coupling parameters; and 4) the very far field, where fracture flow occurs, on average, in an equivalent porous medium.

Our study can be related to the near field flow - assuming that the details of the flow path are known from some type of images of the fractures. Developments in laboratory imaging of core samples (David, 1993; Fredrich, 1993; Fredrich et al., 1995; Lindquist et al., 1996; Doughty and Tomutsa, 1997) provides us with useful means of obtaining sufficient details of the flow paths of reservoir rock samples in order to model the flow in a single fracture at a pore (micro) scale. 


\subsection{Explicit Discrete Fracture Formulation}

Several investigators have published numerical models incorporating explicit discrete representations of fractures. Like Travis (1984), most of the models incorporate fractures explicitly, but these models are restricted to fractures with vertical or horizontal orientation. The advantage of explicit discrete-fracture models is that they allow for explicit representation of fluid potential gradients and fluxes between fractures and porous media with minimal non-physical parameterization. But the fact that fractures may be very tortuous and may have significant impacts on the characterization of flow is often unaccounted for in these models. The numerical model developed in our study is an attempt to allow analysis of flow through fractures of any arbitrary orientation and shape.

\subsection{Dual-Continuum Formulation}

Dual-continuum approaches were introduced by Barenblatt et al. (1960) and later extended by Warren and Root (1963). Dual-continuum models are based on an idealized flow medium consisting of a primary porosity created by deposition and lithification and a secondary porosity created by fracturing, jointing, or dissolution. The basis of these models is the observation that unfractured rock masses account for much of the porosity (storage) of the medium, but little of the permeability (flow). Conversely, fractures may have negligible storage, but high permeability. The porous medium and the fractures are envisioned as two separate but overlapping continua. Fluid mass transfer between porous media and fractures occur at the fracture-porous medium interface. In some numerical approaches, the mass transfer is lumped at the nodes common to the fracture and porous medium grids. The transfer occurs according to a fluid-potentialdependent coupling parameter. This approach averages, or "smears," the transient response between fracture and porous medium.

\subsection{Discrete Fracture Network}

Discrete fracture network (DFN) models describe a class of dual-continuum models in which the porous medium is not represented. Instead, all flow is restricted to the fractures. This idealization reduces computational resource requirements. Fracture "legs" are often represented as lines or planes in two or three dimensions.

Our approach in this study is different from that of the dual-continuum formulations, but conceptually similar to discrete fracture network models. In our study, we treat fracture like a continuous porous media without any granular material embedded in it. Unlike the dual-continuum formulations, our study allows flow only through fractures, which are surrounded by an impermeable, non-porous rock matrix.

\subsection{Statistical Models for Fractures}

In a series of papers, M. Oda and coworkers presented a statistical approach to describing and modeling the elastic deformation and fluid flow properties of fractured rocks (Oda, 1982; Oda, 1985; Oda et al., 1987). The tensors for each physical property are derived by taking a volume average of the expected effect of each fracture in the population. The volume average contains functions of the fracture orientation, length, and aperture in such a way that long fracture or wide fractures contribute relatively more than their smaller cousins.

Oda (1982) envisioned that a general geometric property of cracked rock, termed the fabric, determines many mechanical properties of geological materials. He developed a mathematical description, a fabric tensor, which considers the following elements of crack geometry: 1) position and density of cracks; 2) shape and dimension of cracks, and 3) orientation of rocks. One of the primary simplifying assumptions in the derivation of the fabric tensor concerns the relative position of fractures. Fractures are assumed to have a random position in the network, and all fractures are distributed uniformly throughout the network, as is often described by a Poisson point process. For example, the exact position of any one fracture with respect to any other is not taken into account. Only the length, orientation, aperture, and stiffness of each fracture are retained in the calculation. Thus, highly conductive clusters of fractures will 
not affect the Oda analysis but may in reality have significant effects on fluid flow. In Oda's model, fractures in the network can be arranged in an infinite number of different ways and still have the same mechanical and fluid flow properties. In our study, we emphasize building a simulation tool that can help us understand the fluid properties of different statistical models of fractures that can be constructed.

Oda (1985) developed a tensor model for the fluid permeability of fractured rock. An assumption required for fluid flow in his derivation requires that the fractures be comprised of smooth parallel plates with a constant separation, or aperture $h$. In that case, fluid flow is described by the parallel plate model, where the volumetric flow rate is proportional to the aperture raised to the third power $\left(h^{3}\right)$. A small variation in aperture results in large variations in fluid permeability (Brown and Bruhn, 1998).

The parallel plate model for fluid flow can only be considered a qualitative description of flow through real fractures. Real fracture surfaces are not smooth parallel plates but are rough and contact each other at discrete points. Fluid will take a tortuous path when moving through a real fracture; thus deviations from the parallel plate model are expected. This is our goal in this study - to simulate fluid flow and visualize its motion affected by tortuousity and varying geometry. It is worth noting that further empirical studies showed a relationship between aspects of the crack tensor and the anisotropy of acoustic wave velocities in laboratory specimens (Oda et al., 1986). This result suggests the potential to develop an inverse method to derive important aspects of fracture geometry from geophysical methods. Hence the importance and future prospect of a simulation tool that would handle the details and complexities of a real fracture and model the flow of reservoir fluids through it is not trivial.

Conventional reservoir flow simulation has its roots in the macroscopic description of fluid flow through Darcy's equation. The mathematical fundamentals of reservoir simulators are based on the substitution of Darcy's equation into the mass balance equation for a system and obtaining expressions for pressure in discrete grid-blocks within that system. The pressure equations contain some terms that are related to the rock and fluid properties. One of the most important, and probably the most uncertain among all is called the transmissibility - a lumped parameter that retains information about the property of the porous medium, the fluid flowing through the medium, the direction of flow, and the position in space.

Because of the tortuousity of the flow paths, the actual fluid velocity will vary from point to point within the rock. But the velocity used in Darcy's equation (often referred to as Darcy velocity) is actually an average velocity over the core, and hence the conventional simulators are limited in scope to analyze the flow at the microscopic (pore) scale. Because actual velocities are difficult to measure, they are rarely used in reservoir-engineering calculations. However, in order to understand fluid flow in fractured media, we need to analyze its motion and hence have a realization of the variation of its velocity at a pore scale. Therefore, the equations used in our study must be different than the conventional reservoir simulation equations.

Another problem with conventional reservoir simulation is how the rock permeability, to be used in the transmissibility term, is assigned to each grid-block. Simulators often use a-priori knowledge of the permeability and is rarely derived rarely from the transmission or conductive capacity of actual flow paths in a particular grid block. The conventional methods sometimes cannot incorporate the proper anisotropy information in the flow model, which results into inaccurate flow simulation. For more accurate reservoir simulations, and particularly for identifying high potential production zones in fractured reservoirs, a a conventional approach of assigning the permeability without having correlated with actual flow paths must be changed. Hence we need tools to visualize and improved models to analyze flow in complicated network of fractures to understand the pattern of flow and thus step forward towards finding more accurate ways of tying permeability information to reservoir simulators. Our study is devoted to this cause: understanding the flow and how it is affected by the geometry of the flow paths at a microscale.

\section{$2 \quad$ Mathematical Model}

The motion of a continuous medium is governed by the principle of classical mechanics and thermodynamics for the conservation of mass, momentum, and energy. Fluids in a reservoir, as elsewhere, obey these principles; their flow can be modeled with equations that balance these intrinsic physical properties within a region of investigation. A brief discussion of the mathematical equations and the underlying assumptions that are generally used to describe the basic physics of fluid flow at a microscopic level in a medium is presented in the following. 
In this study, we will assume that the fluid is incompressible and Newtonian. Among the petroleum reservoir fluids, gas-free oil and water can be treated as incompressible fluids under typical reservoir conditions. The equation of motion for a viscous, incompressible, Newtonian fluid is given by the Navier-Stoke's equation:

$$
\rho\left[\frac{\partial \mathbf{V}}{\partial t}+(\mathbf{V} \bullet \nabla) \mathbf{V}\right]+\nabla P-\mu \nabla^{2} \mathbf{V}=\mathbf{f}_{e}
$$

where $\mathbf{V}$ and is the velocity vector and $P$ is the pressure in the moving fluid at each point; $\mathbf{f}_{\mathrm{e}}$ is the external force per unit volume acting on the flowing fluid; $\rho$ and $\mu$ are density and dynamic (or absolute) viscosity of the fluid, respectively; and $\nabla$ and $\nabla^{2}$ are the divergence and the laplacian, operator respectively.

Flow of oil or water in reservoirs, especially in narrow fractures, can be considered as laminar. Furthermore, it is reasonable to assume that the flow is sufficiently "slow" that inertial effects need not be considered in arriving at a solution of the equations of motion. The relative importance of inertial and viscous effects is determined by a dimensionless number, known as the Reynolds number, $N_{\mathrm{Re}}$, defined as:

$$
N_{\mathrm{Re}}=\frac{\text { fluid density } \times \text { speed } \times \text { size }}{\text { viscosity }}
$$

In slow viscous flows, $N_{\mathrm{Re}}$ is small because viscous forces arising from shearing motions of the fluid dominate over inertial forces associated with acceleration or deceleration of fluid particles. Hence, in describing the motion of fluid through fractures in reservoirs, the inertial terms, $\rho(\mathbf{V} \bullet \nabla) \mathbf{V}$, can be omitted to give the governing equation of motion for the so-called creeping flow, known as the Stoke's equation. In the absence of any body forces, the time-dependent Stoke's equation has the following form:

$$
\frac{\partial \mathbf{V}}{\partial t}=-\frac{1}{\rho} \nabla P+\eta \nabla^{2} \mathbf{V}
$$

where $\eta=\mu / \rho$, is the kinematic viscosity of the fluid.

In the absence of any source/sink inside the system, the equation of mass balance is known as the equation of continuity, and for an incompressible fluid it is expressed as:

$$
\nabla \bullet \mathbf{V}=0
$$

Equations (2) and (3) together provide a description of motion of a (single-phase) fluid through porous-permeable media. Single-phase flow through fractures can also be modeled by this set of equations. For some simple cases, such as the flow through a single fracture that can be approximated as flow through parallel plates, analytical solutions to the Stoke's equation exist. However, when the fracture geometry deviates from the idealization, as observed in the real reservoirs, numerical solutions to these partial differential equations are necessary. Numerical models then need to be constructed to simulate the flow and solve for the pressure and velocity variations with space and time within the fracture.

\section{$3 \quad$ Numerical Model}

To solve equations (2) and (3) numerically, methods such as finite difference (Pozrikidis, 2001; Fletcher, 1991; Hirsch, 1988; Kim and Moin, 1985; Patankar, 1980), and finite element (Peyret and Taylor, 1983; Thomasset, 1981; Chung, 1978) can be applied. In some recent studies, cellular automata have been used to obtain solutions for Navier-Stoke's equation in porous media (Ferreol and Rothman, 1995; Frisch et al., 1986). In our study, finite difference approximation is used. Pressure and the scalar components of velocity in the partial differential equations (2) and (3) can be represented in discrete forms for both space and time through equivalent finite difference equations. Using a forward difference scheme for time derivative, the discrete time finite difference representation of equations (2) and (3) is as follows:

$$
\frac{\mathbf{V}^{n+1}-\mathbf{V}^{n}}{\Delta t}=-\frac{1}{\rho} \nabla P^{n+1}+\eta \nabla^{2} \mathbf{V}^{n+1}
$$




$$
\nabla \bullet \mathbf{V}^{n+1}=0
$$

where, $\Delta t$ is the time interval, and $\mathrm{n}=0,1,2,3 \ldots$ is the discrete time step. It is important to note that the spatial discretizations of variables on the right-hand side of equation (4) and on equation (5) are evaluated at the $n+l^{\text {th }}$ time step instead of the $n^{\text {th }}$ time step. For a time-varying finite difference equation, spatial discretization of a variable at an advanced time step is known as the implicit scheme. The physical significance of this implicit representation of the above is such that a condition is imposed on the velocity to obey the continuity principle at the advanced $n+1^{\text {th }}$ time step, while its change over time, from the time step $n$ to the step $n+1$, is computed from the implicit discretization of the Stoke's equation. The fluid on its path will experience change in pressure due to the path geometry and the existing pressure gradient between the inlet and outlet, and as it moves on its velocity vectors will change depending on the pressure. Equation (4) allows the particles to adjust their velocities and pressure while in motion; equation (5) ascertains that the principle of continuity is obeyed by the particles at the end of any discrete movement.

Flow problems that have solutions, which are independent of time and dependent on space only, are called steady state. In the case of incompressible fluid flow through fractures, the effect of fracture apertures and flow path on the motion of fluid can be better understood from the steady-state flow simulation. Hence, we are only interested in the steady-state solution of pressure and velocity in the flow regime. For the numerical formulation that we proposed, the system can be said to have reached steady state when the maximum change of velocity vector between two time steps drops below a very small number. Computationally it means to terminate the iterations when the following criteria for the maximum norm of velocity change is satisfied:

$$
\left\|\mathbf{V}^{n+1}-\mathbf{V}^{n}\right\|_{\infty} \mid<\varepsilon
$$

where $\varepsilon$ is a threshold value defined depending on the desired accuracy of the solution. When this condition is satisfied, the left-hand side of equation (4) becomes (approximately) zero, and the steady-state Stoke's equation is recovered:

$$
\nabla P=\mu \nabla^{2} \mathbf{V}
$$

Our problem requires simulation of fluid flow in rock fractures driven by an applied pressure gradient between the two ends. Initially when there is no fluid in the fracture, the velocity field is zero within the control volume. This is the initial condition of the difference equation: at $n=0, \mathbf{V}^{0}=0$. The fluid can be thought to enter the simulation region (or control volume) through the inlet with a velocity that is initially unknown to us. Then as it moves further in the medium, the motion of the fluid will depend on the pressure gradient that it experiences. Hence, the velocity field will change spatially and with time as the fluid moves from one end to another. Thus we need to derive expressions for pressure and velocities that would model these interactions and changes. For this purpose, we start with taking the divergence of both sides of equation (4):

$$
\frac{\left(\nabla \bullet \mathbf{V}^{n+1}\right)-\left(\nabla \bullet \mathbf{V}^{n}\right)}{\Delta t}=-\frac{1}{\rho} \nabla^{2} P^{n+1}+\eta \nabla^{2}\left(\nabla \bullet \mathbf{V}^{n+1}\right)
$$

Substituting equation (5) into equation (8), the following is obtained:

$$
\nabla^{2} P^{n+1}=\frac{\rho}{\Delta t}\left(\nabla \bullet \mathbf{V}^{n}\right)
$$

Again, equation (4) can be re-arranged to obtain the following form:

$$
\mathbf{V}^{n+1}-(\eta \Delta t) \nabla^{2} \mathbf{V}^{n+1}=\mathbf{V}^{n}-\frac{\Delta t}{\rho} \nabla P^{n+1}
$$

Starting with initial condition $\mathbf{V}^{n}=\mathbf{V}^{0}$, an update of pressure, $P^{n+1}$, is computed from equation (9). Then equation (10) is used to compute the updated velocity, $\mathbf{V}^{n+1}$. The new velocity, $\mathbf{V}^{n+1}$ is then used as $\mathbf{V}^{n}$ for the next step, and updated values for pressures and velocities thereafter are computed once again from equations (9) and (10) respectively. Thus, iterations continue until a solution for velocities is reached for which the inequality condition (6) is satisfied. When inequality (6) is satisfied at the $K^{\text {th }}(K=n+1)$ step, $P^{K}$ and $\mathbf{V}^{K}$ give the desired steady state solution for pressure and velocity, respectively. 
Scalar forms of equations (9) and (10) are used for computation purposes. In this study we define the problem and limit the solutions in 2-D. This method can be applied to 3-D problems as well. Here we use Cartesian coordinates $x$ and $y$; and the components of velocity vector $\mathbf{V}$ in these two perpendicular directions are given by $u$ and $v$,respectively (Figure 1a). For spatial discretization of the equations, we use staggered grids (Figure 1b), where $\mathrm{P}$ is defined at the center of each finite difference grid-cell, and the velocity components $u$ and $v$ are defined on the faces of each cell in a way such that they are perpendicular to each face. The spatial discretization spacing $(\Delta x$ and $\Delta y)$ and the variable indices at the discrete steps used in two perpendicular directions are shown in Figure 1c.

Using central difference approximation for spatial derivatives of both $P$ and velocity components $u$ and $v$, the finite difference form of equation (9) is as follows:

$$
\frac{P_{i-1, j}^{n+1}-2 P_{i, j}^{n+1}+P_{i+1, j}^{n+1}}{(\Delta x)^{2}}+\frac{P_{i, j-1}^{n+1}-2 P_{i, j}^{n+1}+P_{i, j+1}^{n+1}}{(\Delta y)^{2}}=\frac{\rho}{\Delta t}\left[\frac{u_{i+\frac{1}{2}, j}^{n}-u_{i-\frac{1}{2}, j}^{n}}{2\left(\frac{\Delta x}{2}\right)}+\frac{v_{i, j+\frac{1}{2}}^{n}-v^{n}{ }_{i, j-\frac{1}{2}}}{2\left(\frac{\Delta y}{2}\right)}\right]
$$

Further simplification and rearrangement of terms of equation (11) yields the following:

$\alpha P_{i, j+1}^{n+1}+P_{i-1, j}^{n+1}-2(1+\alpha) P_{i, j}^{n+1}+P_{i+1, j}^{n+1}+\alpha P_{i, j-1}^{n+1}=\frac{\beta}{\Delta x}\left(u_{i+\frac{1}{2}, j}^{n}-u_{i-\frac{1}{2}, j}^{n}\right)+\frac{\beta}{\Delta y}\left(v_{i, j+\frac{1}{2}}^{n}-v_{i, j-\frac{1}{2}}^{n}\right)$

where, $\alpha=\frac{(\Delta x)^{2}}{(\Delta y)^{2}}$, and $\beta=\frac{\rho(\Delta x)^{2}}{\Delta t}$ are constants.

Equation (12) is applied to each of the finite difference grids $(i, j)$ and a system of equations is obtained which can be expressed in matrix notation as follows:

$$
\underline{\underline{\mathbf{A}}} \underline{\boldsymbol{P}}=\underline{\boldsymbol{d}} \ldots
$$

where $\underline{\boldsymbol{P}}$ is the unknown $m \times 1$ vector ( $\mathrm{m}$ is the total number of grid-cell center points where the pressure $P$ is defined), $\underline{\underline{\mathbf{A}}}$ is the $m \times m$ coefficient matrix, and $\underline{\boldsymbol{d}}$ is the known $m \times 1$ vector (since the quantities on the right hand side of equation (12) are evaluated at the present time step $n$, they are known). Depending on the size of the grids and the available computer power, direct or iterative solution methods (Hirsch, 1988) can be used for solving the system given by equation (13). Commonly used direct methods are forward, backward substitution, and LU decomposition. Commonly used iterative methods are Jacobi, GaussSeidel, SOR, and multigrid. If iterative solutions are required, different acceleration and convergence techniques, e.g. Krylov-subspace methods (Kasenally, 1992; Saad, 1981) can be applied to improve the solution. Once $P^{n+1}$ at all the grid points are computed from equation (13), they are used in the computation of $u^{n+1}$ and $v^{n+1}$ from the scalar form of equation (10). Since these variables are staggered in space, they are not evaluated at the same points of the grids. However, this is not a major source of error, as the finite difference schemes applied to the partial derivatives of all the variables are consistent and congruous with each other. Moreover, a simple averaging technique can bring them all to the same point. For example, the following averaging can be done to compute a single velocity for an individual grid-cell:

$$
\begin{gathered}
u_{i, j}=\frac{u_{i+\frac{1}{2}, j}+u_{i-\frac{1}{2}, j}}{2} \\
v_{i, j}=\frac{v_{i, j+\frac{1}{2}}+v_{i, j-\frac{1}{2}}}{2}
\end{gathered}
$$

The $n+l^{\text {th }}$ step solutions for $u$ and $v$ in the entire flow regime are computed from the scalar finite difference approximations of equation (10). The total number of $u$ 's and $v$ 's to solve for will not only depend on the discretization step size, but also on the pattern of connection of the grid-cells. One of the limitations of our finite difference scheme is that it has to approximate the curved boundaries of the fractures with smallest 
possible straight line segments; the entire simulation region will be comprised of rectangular grid cells with the smallest possible size. On this matrix of tiny cells, if there are $r$ number of connected cells in a row, then we will have $r+1$ number of $u$ 's to solve for that row of connected cells. Similarly, if a column has $c$ number of connected cells, then we will have $c+1$ number of $v$ 's to solve for that column of connected cells. Applying central difference schemes at each face of the cells separately, the finite difference approximation of the scalar components of equation (10) yields systems of equations similar to equation (13) for both $u$ and $v$. An example of how this discretization is carried out is given in the following, where the $x$-component of equation (10) is applied to a point $\left(i+\frac{1}{2}, j\right)$ on the edge of a cell where $\mathrm{u}$ is defined and the $y$-component of equation (10) is applied to a point $\left(i, j-\frac{1}{2}\right)$ on the edge of a cell where $v$ is defined:

$$
\begin{aligned}
& \left.\alpha u_{i+\frac{1}{2}, j+1}^{n+1}+u_{i-\frac{1}{2}, j}^{n+1}-[2(1+\alpha)+\gamma]\right]_{i+\frac{1}{2}, j}^{n+1}+u_{i+\frac{3}{2}, j}^{n+1}+\alpha u_{i+\frac{1}{2}, j-1}^{n+1}=-\gamma u_{i+\frac{1}{2}, j}^{n}+\frac{\Delta x}{\mu}\left(P_{i+1, j}^{n+1}-P_{i, j}^{n+1}\right) \\
& \alpha v_{i, j+\frac{1}{2}}^{n+1}+v_{i-1, j-\frac{1}{2}}^{n+1}-[2(1+\alpha)+\gamma] v_{i, j-\frac{1}{2}}^{n+1}+v_{i+1, j-\frac{1}{2}}^{n+1}+\alpha v_{i, j-\frac{3}{2}}^{n+1}=-\gamma v_{i, j-\frac{1}{2}}^{n}+\frac{\Delta y}{\mu}\left(P_{i, j}^{n+1}-P_{i, j-1}^{n+1}\right)
\end{aligned}
$$

where, $\gamma=\frac{(\Delta x)^{2}}{\eta \Delta t}$ is a constant.

\section{Boundary Conditions}

The flow we want to simulate is through rock fractures of any known geometry. Physically the flow is bounded by no-flow solid boundaries or rigid walls everywhere, except for some openings for the fluid to enter the system (termed as inlet) and to leave the system (termed as outlet). The $(x, y)$ positions and geometry of the inlet(s) and outlet(s) have to be known, and they may be situated anywhere on the system boundaries. There is no additional source or sink within the system and a continuous stream of flow is maintained through the system so that a steady-state situation can be simulated.

Mathematically, the following boundary conditions can be posed for our problem, as shown in Figure (2):

a) $u=v=0$ at the no-flow boundaries.

b) $P=P_{a}$ at inlet/outlet $a$.

However, for the purpose of numerical computation with central finite difference methods, these boundary conditions are not enough. When the finite difference equations (12), (16), and (17) are applied to the grid cells that have at least one face coinciding with the physical boundary of the system, terms outside the flow region appear in the difference equations which do not exist physically. As discussed earlier, finite difference equations, as we apply to all the grid-cells in an orderly fashion in our solution method, will generate a system of equations similar to equation (13) for each of the variables. However, it is required that the coefficient matrix be square for a well-posed problem. In order to fulfill this requirement we need to find additional conditions for those additional nonexistent terms so that more equations can be added to make the number of equations equal to the number of unknowns in the system of equations. This task is often accomplished by superimposing imaginary nodes outside the boundary, which is often referred to as reflection points or ghost-grids or image-grids (Aziz and Settari, 1979).

\subsection{BC at Fracture Walls}

The rigid walls in our problem, which prevents cross flow of fluid between the fracture and rock matrix, exhibit a no-slip boundary condition, a condition that requires that the fluid in contact with a container's 
wall be at rest. These no-flow rigid walls in our 2-D problem are restricted in orientation so that they lie along the boundaries of the finite-difference grid cells. A vertical wall therefore passes through the horizontal velocity $(u)$ mesh points, and the $u$-velocities at those points vanish at all times $(u=0)$. In our staggered grid formulation, a vertical wall does not pass through vertical-velocity $(v)$ mesh points, but the calculation requires the values of $v$ at mesh points lying just outside of the wall, as explained earlier. If $v^{\prime}$ is denoted as the vertical velocity beyond the rigid wall, then for a no-slip wall the boundary condition is $v^{\prime}=-v$ (Harlow and Welch, 1965). Figure 3(a) summarizes the numerical boundary conditions around a vertical wall. Since the vertical velocities are simply reversed across the wall $\left(v=-v^{\prime}\right)$ and $\nabla \bullet \mathbf{V}=0$ in the fluid cell, it follows that the vanishing of $\nabla \bullet \mathbf{V}^{\prime}$ (divergence of velocity in the imaginary cell right outside the wall) is accomplished only if $u^{\prime}=+u_{1}$.

Analogous boundary conditions for velocity are applied at a horizontal wall (Figure 3(b)). To summarize the velocity boundary conditions at no-flow boundaries it can be stated that for a no-slip wall, normal velocity remains the same, while tangential velocity reverses.

Wall boundary conditions for pressure are also needed for the numerical solution of the pressure equation (12). While it is not necessary to calculate the changes in normal velocity for points lying on the wall, the boundary conditions for $P$ must be consistent with the identical vanishing of that velocity. The relationship between pressures outside the wall (in imaginary grids) and in the flow region is found by substituting velocity boundary conditions on equations (16) and (17), which are simply the discretized scalar forms of the Stoke's equation (equation 4).

For a vertical wall (as shown in Figure 3a), the substitution on equation (16) yields the following:

$$
P^{\prime n+1}=P_{1}^{n+1} \pm 2 \mu \frac{u_{1}^{n+1}}{\Delta x}
$$

where the sign is "+" for fluid to the left of the wall and "-" for fluid to the right of the wall. For a no-slip wall that is horizontal, pressures are related as:

$$
P^{\prime n+1}=P_{1}^{n+1} \pm 2 \mu \frac{v_{1}^{n+1}}{\Delta y} \ldots \ldots
$$

A difficulty arises in implementing this pressure boundary condition to the numerical solution scheme that we devised. The starting point of our numerical solution is equation (12); in each time-step the velocity can be updated only after an update in pressure is computed. However, equations (18) and (19) require that the pressures in the ghost-grids beyond the wall are related to that in the fluid side by velocity of fluid at the same time instance. The way we treat the update of each variable at a time through separate equations makes it impossible to have a-priori knowledge of velocity at $n+1^{\text {th }}$ time step when we compute pressure at $n+l^{\text {th }}$ time step. To circumvent this problem and simplify our numerical computations, we first consider the fact that velocity in a unidirectional Stoke's flow has an analytic solution that resembles a parabola. From the parabolic velocity profile as the one shown in figure (4), it is reasonable to infer that the fluid particles very close to the boundary are almost stationary. Provided that our grids are sufficiently small, the errors generated due to an approximation of vanishing near wall velocities $\left(u_{1} \approx 0 ; v_{1} \approx 0\right)$ only when an expression for $P^{\prime}$ needs to be found, would be negligibly small in a high-resolution finite difference scheme. Hence, at any time step $K$, for computational purposes, we can make use of the following relationship:

$$
P^{\prime}=P_{1}
$$

This is in fact a discrete form (using central difference formula) of a very well known boundary condition used in reservoir engineering: $\frac{d P}{d s}=0$, where $s$ is the normal to the boundary wall. In reservoir simulation, by specifying a pressure gradient normal to the boundary (Neumann type BC), one can prescribe the flux (or velocity) normal to the boundary. A special case often encountered is the no-flow boundary where the flux vanishes everywhere on the boundary. If flow across the boundary does not exist, this implies that the pressure gradient across the boundary is also zero (Ertekin et al., 2001; Aziz and Settari, 1979) and can be easily proved from Darcy's equation. A volumetric reservoir with completely sealed outer boundaries is equivalent to a zero pressure gradient across its outer boundaries. This is exactly 
the same as in our case, where we have fluids neither entering, nor leaving the system through the solid/noslip boundary walls. Hence, apart from being a source of some infinitesimal and insignificant numerical error, equation (20) is a valid and appropriate condition to use at the solid wall (no-flow) boundaries in our problem.

\subsection{BC at Inlet and Outlet}

Specifying the pressure boundary conditions at the inlet and outlet is straightforward. Constant pressures are prescribed for inlets and outlets (Figure 5). This type of boundary condition, known as Dirichlet type $B C$, occurs in reservoirs that are constantly charged by a strong water influx so that the pressure at the interface between the hydrocarbon reservoir and the supporting aquifer remains constant. In our problem the constant pressures are maintained at the inlets and the outlets, which can be thought to remain connected with constant pressure reservoirs/sources/sinks.

Assuming that we do not know the inlet and outlet flow rates from which we can stipulate the velocity boundary conditions at these nodes, we put some restriction as to how fluid can enter or leave the system. As we faced at no-flow boundaries, inlets and outlets also require expressions for variables at ghost/imaginary cells, either numerical or some relation with those at the real cells in the control volume. We assume that the fluid enters and leaves the system, respectively, in directions normal to the inlet and outlet. This allows us to equate the tangential velocity components in the imaginary cell outside the control volume to zero. Hence, to satisfy continuity in the imaginary cell, the normal component has to remain unchanged within that cell, and from that we obtain suitable expressions to substitute for ghost-cell velocities in our difference equations at the inlets and outlets.

\section{Computer Model}

A computer program, ERLFRAC, is developed to simulate fluid flow through rock fractures by computing pressure and velocity at closely spaced grid-cells. The code requires a description of the flow path and it overlays a finite difference grid on it, regardless of the geometrical complexity of the flow path. A limitation in this is that it approximates the curved boundaries with linear sections; however, the higher the grid resolution, the finer the boundary discretization and the lesser the difference between real and numerical boundaries. The computer program can also accept as input binary image of core samples with rock matrix and pore spaces identified. The program also requires specifying the inlet and outlet locations; it can handle multiple inlets and outlets anywhere on the boundary. The static pressures at the inlets and outlets also need to be specified. The termination criteria, $\varepsilon$, has to be provided, and depending on the accuracy need, it may be changed from problem to problem. Having known all these, the program starts its iterations with initial $\mathbf{V}=0$ and continues until condition (6) is satisfied. Apart from computing velocity vectors and pressures at each iteration, the program also monitors the amount of mass flow in and out of the system. As we do not have any fluid injection or production interior to our system, it is imperative that there is no net accumulation in the system (mass in-mass out $\approx 0$ ). Finally, the program plots out the steady-state solution - it shows the fluid motion in the pressure field. The velocity components in each direction along the fracture are also plotted out. The plots generated by the computer program serve an important purpose: a better understanding of flow in fractures through visualization.

Currently ERLFRAC is capable of simulating flow in two dimensions. Although real flows are in three dimensions, 2-D studies are often of great importance because they are relatively simpler to model and are very effective in understanding and evaluating the solutions. Work is in progress to extend this computer program to make it a complete micro-scale 3-D flow simulation package in fractures of known geometry. The numerical model will conceptually remain the same, except there will be an addition of another velocity component and pressure gradient to the existing set of equations. Computationally, it will increase the efforts of bookkeeping and may significantly increase the need of computer power.

\section{Model Validation}

The computer model developed for this study is used to simulate flow through different fracture-like geometries in two dimensions. The program solves for velocity vectors and pressure in the flow field. As 
for fluid properties, $\mu=2 c p$ and $\rho=0.8 \mathrm{~g} / \mathrm{cc}$ are used. For all the cases studied, spatial grids $\Delta x=\Delta y=$ $1 \times 10^{-4} \mathrm{~m}$ are used. For time discretization, $\Delta t$ for each problem is chosen separately, such that relatively faster convergence to steady state can be obtained with meaningful velocity values allowing for very small percentage error in mass continuity. Depending on the overall accuracy of solutions required for each case and looking at the order of magnitudes of velocities and convergence rates in individual problems, different termination conditions $\varepsilon$ are applied to different cases.

The simplest fracture geometry that can be considered is probably a region bounded by two parallel plates (Figure 4). We consider a unidirectional flow between the parallel plates with known height/spacing (fracture aperture $h$ constant along its length $L$ ). We assume that both the plates are fixed (no-slip boundaries) and a steady flow is set up by a constant applied pressure gradient. Analytical solutions of Stoke's equation (Fox and McDonald, 1998) exist for this type of set-up. The velocity in the $y$ direction $(v)$ is zero, and the velocity in the $x$-direction $(u)$ is a function of an applied pressure gradient in the $x$-direction and its distance from the boundary walls:

$$
u=-\frac{1}{2 \mu} \frac{d P}{d x}\left[\frac{h^{2}}{4}-\left(y-\frac{h}{2}\right)^{2}\right]
$$

The velocity profile is thus parabolic (Figure 4). The flow is fastest in the center and zero on the sidewalls. The areal flow rate (equivalent to volumetric flow rate in $3-\mathrm{D}) Q$, and the mean velocity $\bar{u}$ in this case can be computed as:

$$
\begin{aligned}
& Q=\int_{o}^{h} u d y=-\frac{h^{3}}{12 \mu} \frac{d P}{d x} \\
& \bar{u}=\frac{1}{h} \int_{0}^{h} u d y=-\frac{h^{2}}{12 \mu} \frac{d P}{d x}
\end{aligned}
$$

In order to test whether our numerical model can produce a solution that agrees well with the available analytic solution of Stoke's equation, a test simulation is carried out on a parallel plate type fracture geometry. A constant pressure gradient $(\mathrm{dP})$ of $10 \mathrm{kPa}$ is applied over a $500 \times 10^{-4} \mathrm{~m}$ long fracture that has a constant aperture of $20 \times 10^{-4} \mathrm{~m}$ along its entire length. The visual description of the flow through this fracture is given in Figure (6). It is observed that the velocity component in the $x$-direction has a parabolic shape with velocity maximum at the centerline and gradually decreasing towards the boundary walls. For the given fracture length and aperture, an analytical solution predicts maximum $u$ to be $50 \mathrm{~m} / \mathrm{s}$ and our simulation computes maximum $u$ as $49.95 \mathrm{~m} / \mathrm{s}$. The analytical and our computed values of mean $u$ are $33.333 \mathrm{~m} / \mathrm{s}$ and $33.433 \mathrm{~m} / \mathrm{s}$, respectively. Velocity component in the $y$-direction is theoretically zero in this case and our computation also yields that result. Analytical and computed values for $Q$ are both $6.7 \times 10$ ${ }^{2} \mathrm{~m}^{2} / \mathrm{s}$.

Since density is constant in our system, to check if mass is conserved in the system, or in other words to check if mass entering the system per unit time is equal to the mass leaving the system per unit time, the following condition should be satisfied:

$$
\sum Q_{\text {in }}=\sum Q_{\text {out }}
$$

For the simplest geometry, described as in case \#1, equation (23) is exactly satisfied. However, for irregular geometries described later, some errors will be introduced due to different gridding at the inlets and outlets. The percentage error in the mass continuity in our numerical scheme can be defined as:

$$
\frac{\left|\sum Q_{\text {in }}-\sum Q_{\text {out }}\right|}{Q_{\text {in }}} \times 100 \%=q
$$

In our subsequent simulations, we seek solutions such that $q$ is less than $1 \%$.

Our simulation results for case\#1 match the analytic solutions of the problem very well. Moreover, in our numerical scheme the maximum change in $\mathbf{V}$ decreases exponentially with iterations and the 
solutions are highly accurate. Hence, our numerical model is valid and we proceed to use it for simulation of flow in rather complicated fracture networks.

\section{Flow Simulation: Case Studies}

Eight different cases, each representing a different scenario in the reservoir in terms of variation of flow paths, are studied for this paper. The simulation results of the cases obtained from ERLFRAC are given in Figures (6) through (13). The axes of the plots are scaled for the fracture lengths $\left(500 \times 10^{-4} \mathrm{~m}\right.$ for cases \#1 -

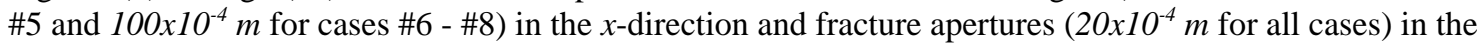
$y$-direction. The reverse $y$-scales are just to facilitate the plots - the models actually follow the cartesian coordinate system adopted in this study (Figure 1a). All the case-specific information, and some significant observations, are listed in Table (1) for comparison and discussions. Also to get an idea about the convergence rate for each problem, additional plots are provided in Figures (14) and (15).

The cases studied here have at least one inlet at the left-most side, and at least one outlet on the right-most side. To enhance the complexity of the flow, we have placed additional inlets and outlets on the upper and lower boundaries in cases \#6, \#7, and \#8. For all the cases, pressure boundary conditions 1000 $\mathrm{KPa}$ are used at the inlets, and $990 \mathrm{KPa}$ are used at the outlets for cases \#1 - \#5, and $995 \mathrm{KPa}$ for cases \#6 \#8. Although for flow simulation of incompressible fluids, pressure difference $\Delta P$ is all that is required. However, the high order of magnitude used here is an attempt to represent real reservoir conditions. In Figures (6) through (13), the pressure variation and velocity variation in the fractures are represented by gradation of color schemes on the top and bottom panels, respectively. The arrows on the top panel in each plot represent the velocity vectors of fluid at discrete points in the fracture - their directions indicate the direction of motion of the fluid and their size indicates the relative magnitude of velocity.

The choice of $\Delta x$ and $\Delta y$ to be used for the numerical scheme depends on the resolution of the solution required. The selection of these FD spatial discretization parameters is often a tradeoff between required resolution and available computer power. For our problems, we chose $\Delta x=\Delta y=1 x 10^{-4} \mathrm{~m}$. We also ran our computer program for some cases with other choices of spatial discretization, although the plots are not shown on this paper. We observed that decreasing $\Delta x$ and $\Delta y$ resulted in better control of mass flux (lower $q$ ) in our numerical scheme, however there was no way of saying how far we needed to decrease the spatial grid sizes in order obtain the best or most accurate solutions for velocity and pressure. This particular issue needs to be studied further.

Another important FD discretization parameter is $\Delta t$. A proper choice of $\Delta t$ is also important for the stability of the numerical scheme. To determine the best $\Delta t$ for our problems, we started with finding a $\Delta t$ such that the following condition is satisfied:

$$
\Delta t \leq \frac{(\Delta x)^{2}}{2 D}
$$

where $D$ is the dimension of the problem. Condition (25) is a generalized stability condition that can be derived by applying Von-Neumann or Fourier series stability analysis on finite-difference schemes of partial differential equations (Hirsch, 1988; Smith, 1978; Lax and Richtmeyer, 1956; DuFort and Frankel, 1953). Then we reduced $\Delta t$ further and for individual problems chose an optimum value of $\Delta t$ for speed of convergence and guarantee of these two conditions -1) maximum change in velocity vectors should gradually decrease with iterations, and 2) $q$, as defined in equation (24) should be less than $1 \%$.

Semilog plots of how the maximum changes in computed velocity and pressure change with iterations in our computational scheme are provided in Figures (14) and (15). The plots for all the cases indicate that our computational model is quite stable, although the convergence speed to required accuracy varies from case to case. It was observed that the maximum absolute change in pressure that could be obtained was in the order of $10^{-13}$. Beyond that accuracy, a steady pressure solution was obtained meaning that the solution for pressure would not improve any further. When the steady pressure values were reached, velocity change was also observed to have ceased, except for case \#1 where velocity kept on changing despite a steady pressure solution was obtained only after five iterations. Numerically, the recurrent velocity change is due to the tendency of the algorithm to construct the finest parabola in each of the tiniest parallel plate sections of the fracture. However, for practical considerations, in most of our problems we terminated the computations after a reasonable accuracy in the solutions were achieved (stipulated by choice of $\varepsilon$ ), rather than waiting till the end. 
As we observe in all the results (Figures 6 through 13), the direction and magnitude of pressure drop controls the direction and magnitude of the flow. Depending on the path geometry, we may have fluid flowing in any direction internally. However, the overall direction of the flow depends on the position of the inlets and outlets and the prevailing pressure gradient between them. Also observed is the effect of inlet aperture to the amount of flow. At the inlet, the amount of fluid entering and its flow velocity would be proportional to the fracture aperture $h$ - the wider the opening, the higher the flow. A good illustration for this is case \#6. The flow is the highest (both in magnitude and in the amount of fluid flux) through the channel that has entrance between $\mathrm{x}=60$ and $\mathrm{x}=72\left(\mathrm{~h}=12 \times 10^{-4} \mathrm{~m}\right)$ compared to the other channels. In terms of total mass flux through the system, flow geometry as in case \#6 has the highest capacity to transmit fluid among all the cases studied, mainly because of this wide channel and a high pressure gradient in the $y$-direction along it, and the existence of multiple inlets on the fractured rock. Another important observation is the preferential flow of fluid through wider channels towards the outlet. For example, in case \#3, fluid has the highest velocity along the center when it enters the rock, but when it encounters three different fracture legs as paths to flow to the outlet, it tends to move to the sides towards the wider legs. All the three legs have the same length and are kept at the same pressures at the outlets, but their apertures vary and the flow rate (or flow velocity) is proportional to the opening $h$. In case \#3, the flow velocity is almost thrice in the side-legs which both have almost three times larger flow opening than the middle one. Such a phenomenon can be helpful in understanding production from fractured reservoirs. Case \#4 reveals another important fact about fluid flow in fractures. Fluids tend to avoid the dead ends, irrespective of the apertures of the dead-end pore spaces. The reason is that the pressure gradient gets distributed in such a way that there is almost no pressure change in those sections of the fracture where the ends do not turn into any outlet. Although these dead-end sections entrap fluids, if there is a fair amount of pressure drop between the inlet and outlet, then fluid could even make drastic change in its course and bypass such dead-ends and flow towards the sections where there is a possible exit.

If there is sudden change of aperture, the fluid readjusts its velocities according to the varying apertures in order to maintain the continuity in the flow. As seen in case \#2, the middle section gets narrower and again widens up further down the stream. There are three different flow apertures along a continuous path, and the velocity values in these different sections reflect the effect of channel crosssections. Velocity of fluid increases by the amount of decrease in flow aperture (cross-section) and vice versa, if the same fluid passes through regions of varying cross-sections along its path. This observation is also supported mathematically, as equation of continuity in fluid dynamics require $Q=\mathbf{V} \bullet \mathbf{A}=$ constant along the entire flow path.

In reservoir simulation, the transmissive property of rock, namely permeability, is expressed through Darcy's equation. Although the set of equations that we use in our micro-scale study do not explicitly contain the term permeability, yet by analogy to Darcy's equation an equivalent conductivity term can be defined for parallel plate models, which is equal to $\frac{h^{2}}{12}$. The concept of computing equivalent/overall conductivity for a network of fractures with each section having geometry like that of a parallel plate model and all the sections in the network being connected in series or in parallel or both ,is very similar to the principle of computing equivalent resistance of electrical circuits. However, in this study, since we tend to deviate from the linear geometry of the fracture network, we do not implement the network models (Madden, 1976; Dullien 1992; Snow, 1969) to compute the overall conductivity of the rock. Unfortunately, neither could we find an appropriate method to facilitate the computation of permeability tensor in micro-scale. Hence, we turn to Darcy's equation in order to analyze the relative conductance for the cases studied. We compute an average permeability $k$ for the fractures as follows:

$$
\begin{aligned}
& k_{x}=-\frac{\bar{u} \mu}{d P / d x} \\
& k_{y}=-\frac{\bar{v} \mu}{d P / d y}
\end{aligned}
$$


where, $k_{x}$ and $k_{y}$ are the average overall permeability in $x$ and $y$ direction respectively. As the position of inlets and outlets may not necessarily fall along a straight line, $\frac{d P}{d x}$ and $\frac{d P}{d y}$ is just an overall pressure change observed between the outlet and inlet over a straight line distance between the two sides where the inlets and outlets are located in either direction. Hence, the magnitudes of $k_{x}$ and $k_{y}$ computed in our examples do not represent the actual conductivity, but can be used to analyze the relative conductance of the different fracture geometries studied.

As expected for fractures, all the computed permeability values (expressed in darcy units; $1 \mathrm{~m}^{2}=$ $1.01325 \times 10^{12} d$ ) turned to be extremely high. Although there are internal flows in both directions in most of the cases studied, we choose to compute and report permeability values only for those directions where there is actual transmission of fluid through the rock. The values obtained suggest that permeability is inversely proportional to the complexity of the flow path. Although the computation (equations 25 \& 26) does not account for the path tortuousity, the computed values, when compared against the path geometries, clearly indicate that permeability is a strong function of the easiness of the flow through a fracture, which is also true intuitively. This also supports the empirical models for permeability proposed by Kozeney (1927) and Carman (1937).

\section{Conclusions}

We have simulated two-dimensional single-phase fluid flow for eight different fractures. The computer program ERLFRAC meets both computational and visualization needs of important fluid flow properties at micro-scale. The simple examples provide good insight into fluid flow in fractures and its dependence of fracture properties.

\section{Acknowledgments}

This work was supported by the Earth Resources Laboratory Founding Members, the Department of Energy Grant numbers DE-FG0s-00ER15041 and DE-FC26-02NT15346, and ENI S.p.A. AGIP. 


\section{References}

Aziz, K., and Settari, A.: Petroleum Reservoir Simulation, Applied Science Publishers Ltd., London. (1979).

Barenblatt, G.E., Zheltov, I.P., and Kochina, I.N.: "Basic concepts in the theory of seepage of homogeneous liquids in fissured rocks," Journal of Applied Mathematics (USSR), 24(5), pp.12861303. (1960).

Bear, J.: "Modeling flow and contaminant transport in fractured rocks," in Flow and Contaminant Transport in Fractured Rock, Bear, J., C.-F. Tsang, and G. de Marsily (editors), Academic Press, New York, N.Y. (1993).

Bear. J., and Berkowitz, B.: "Groundwater flow and pollution in fractured rock aquifers," in Development of Hydraulic Engineering, vol. 4, P. Novak (editor), Elsevier Applied Science, Oxford, England. (1987).

Brown, S.R., and Bruhn, R.L.: "Fluid Permeability of deformable fracture networks", Journal of Geophysical Research, Vol. 103, no. B2, pp 2489-2500. (Feb 10, 1998).

Carman, P.C.: "Fluid flow through granular beds," Trans. Inst. Chem. Engrs., London, 15, 150. (1937).

Chung, T.J.: Finite Element Methods in Fluid Dynamics, McGraw-Hill, New York. (1978).

David, C.: "Geometry of flow paths for fluid transport in rocks," J. Geophys. Res., 98, pp. 12267-12278. (1993).

Doughty, D.A., and Tomutsa, L.: "Imaging pore structure and connectivity in high resolution NMR Microscopy," Int. J. Rock Mech. \& Min. Sci., 34:3-4. (1997).

DuFort, E.C., and Frankel, S.P.: "Stability conditions in the numerical treatment of parabolic differential equations," Math Tables, Natl. Research Council, Washington, DC, 7, 135. (1953).

Dullien, F.A.L.: Porous Media: Fluid Transport and Pore Structure, Second Edition, Academic Press, San Diego. (1992).

Ertekin, T., Abou-Kassem, J.H., and King, G.R.: Basic Applied Reservoir Simulation, SPE textbook series vol. 7, Society or Petroleum Engineers, Richardson, Texas. (2001).

Ferreol, B., and Rothman, D.H.: "Lattice-Boltzmann simulations of flow through Fontainebleau sandstone," Transport in Porous Media, 20, pp. 3-20. (1995).

Fletcher, C.A.J.: Computational Techniques for Fluid Dynamics, Springer-Verlag, New York. (1991).

Fox, R.W., and McDonald, A.T.: Introduction to Fluid Mechanics, $5^{\text {th }}$ ed. J. Wiley, New York. (1998).

Fredrich, J.T., Greaves, K.H., and Martin, J.W.: "Pore geometry and transport properties of Fontainebleau sandstone,” Int. J. Rock Mech. Min. Sci., 30, pp. 691-697. (1993).

Fredrich, J.T., Menendez, B., and Wong, T.-f.: "Imaging the pore structure of geomaterials," Science, 268, pp. 276-279. (1995).

Frisch, U., Hasslacher, B., and Pomeau, Y.: "Lattice-gas automata for the Navier-Stokes equation," Phys. Rev. Lett., 56, pp. 1505-1508. (1986).

Harlow, F.H., and Welch, J.E.: "Numerical calculation of time-dependent viscous incompressible flow of fluid with free surface," The Physics of Fluids, vol. 8, no. 12, pp. 2182-2189. (1965).

Hirsch, C.: Numerical Computation of Internal and External Flows, Vol. 1 \& 2, Wiley \& Sons, New York. (1988).

Kasenally, E.M.: “Analysis of some Krylov subspace methods for large matrix equations," IRC-PSE Report, No. C93-14, Imperial College, University of London. (1992).

Kim, J., and Moin. P.: “Application of a fractional-step method to incompressible Navier-Stokes equations," Journal of Computational Physics, 59, pp. 308-323. (1985).

Kozeny, J.: “Ober kapillare leitung des wassers in boden," S. Bev. Wiener Akad., Abt. Iia, 136, 271. (1927).

Lax, P.D., and Richtmeyer, R.D.: "Survey of the stability of linear finite difference equations," Communications on Pure Applied Mathematics, 9, 267. (1956).

Lindquist, W.B., Lee, S.-M., Coker, D.A., Jones, K.W., and Spanne, P.: "Medial axis analysis of void structure in three-dimensional tomographic images of porous media," J. Geophys. Res., 101, pp. 8297-8310. (1996).

Madden, T.R.: "Random networks and mixing laws,” Geophysics, 41 (6A), pp. 1104-1125. (1976). 
Mok. U., Bernabe, Y., and Evans, B.: "Permeability, porosity and pore geometry of chemically altered porous silica glass," Journal of Geophysical Research - Solid Earth, Vol. 107, No. B1, (January 2002).

Oda, M.: "Fabric tensor for discontinuous geological materials," Soils Found., 22, pp. 96-108. (1982).

Oda, M.: "Permeability tensor for discontinuous rock masses," Geotechnique, 35, pp. 483-495. (1985).

Oda, M., Hatsuyama, Y. and Onish, Y.: "Numerical experiments on permeability tensor and its application to jointed granite at Stripa Mine, Sweden," J. Geophys. Res., 92, pp. 8037-8048. (1987).

Oda, M., Yamabe, T., and Kamemura, K.: “A crack tensor and its relation to wave velocity anisotropy in jointed rock masses,” Int. J. Rock Mech. Min. Sci. Geomech. Abstr., 23, pp. 387-397. (1986).

Patankar, S.V.: Numerical Heat Transfer and Fluid Flow, Hemisphere Publishing Corporation, U.S.A. (1980).

Peyret, R., and Taylor, T.D.: Computational Methods for Fluid Flow, Springer-Verlag, New York. (1983).

Pozrikidis, C.: Fluid Dynamics: Theory, Computation and Numerical Simulation; accompanied by the software library FDLIB. Kluwer Academic. (2001).

Saad, Y.: "Krylov subspace methods for solving large unsymmetric linear systems," J. Mathematics of Computation, Vol 37, pp. 105-126, (1981).

Sahimi, M.: Flow and Transport in Porous media and Fractured Rock: From Classical Methods to Modern Approaches, VCH, Weinheim. (1995).

Smith, G.D.: Numerical Solution of Partial Differential Equations: Finite Difference Methods, Oxford Applied Mathematics and Computing Science Series, Oxford U. Press, Oxford. (1978).

Snow, D.T.: "Anisotropic permeability of fractured media," Water Resources Research, v.5, no.6, pp.12731289. (1969).

Thomasset, F.: Implementation of Finite Element Methods for Navier-Stokes Equations, Springer-Verlag, New York. (1981).

Travis, B.J.: "TRACR3D: A Model of Flow and Transport in Porous/Fractured Media,” LA-9667-MS, Los Alamos National Laboratory, Los Alamos, N.M., (1984).

Warren, J.E., and Root, P.J.: “The Behavior of Naturally Fractured Reservoirs," Society of Petroleum Engineers Journal, pp. 245-255. (Sept. 1963). 


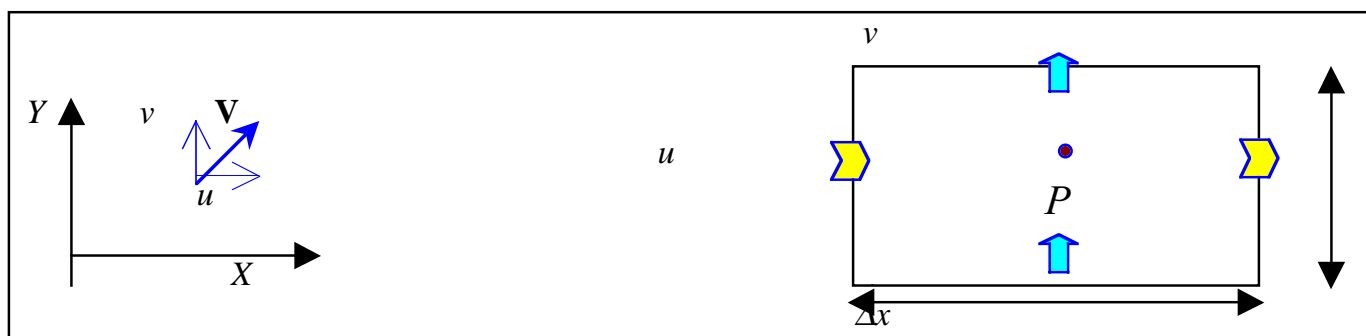

(a)

(b)

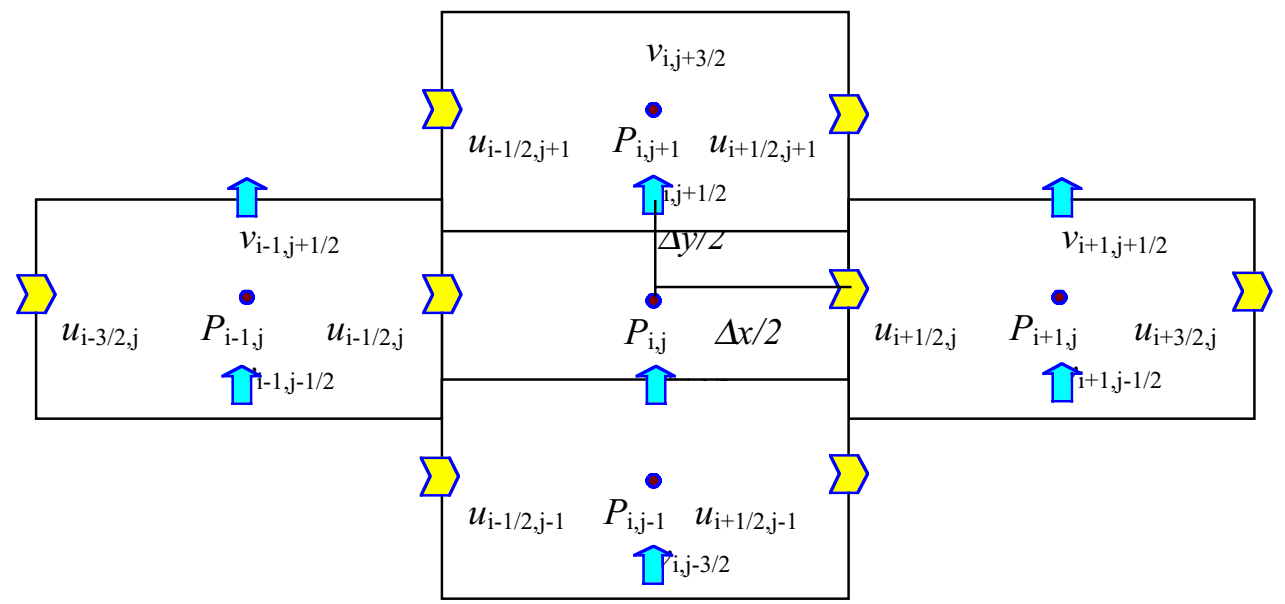

(c)

Figure 1. (a) Coordinate system used.

(b) Use of a staggered grid scheme to define variables.

(c) FD discretization 


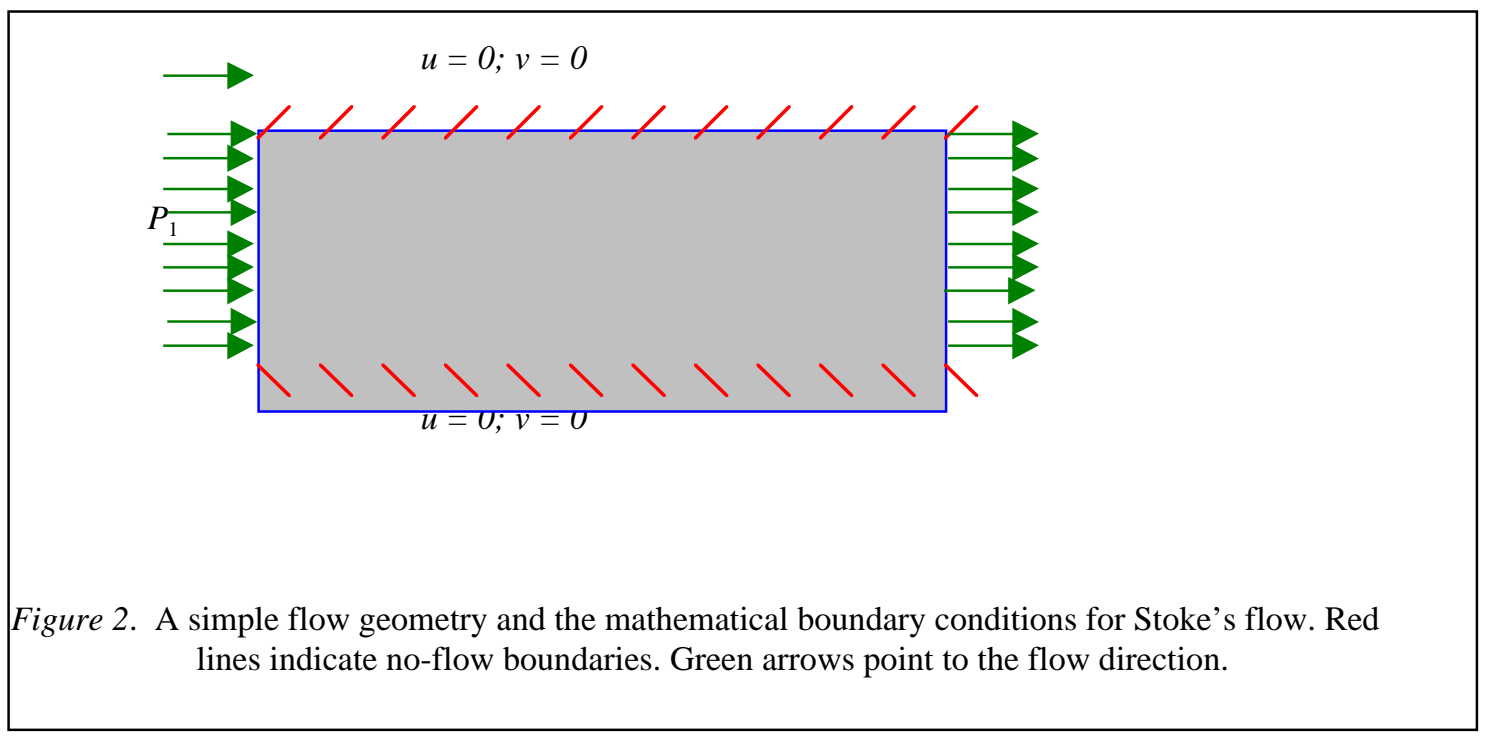




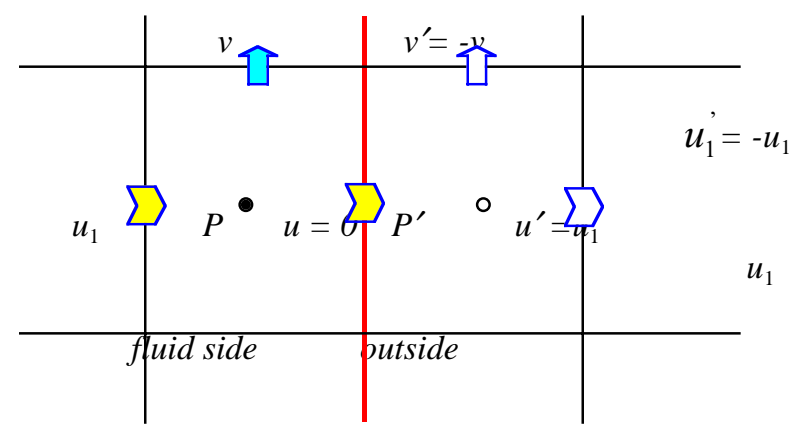

(a)

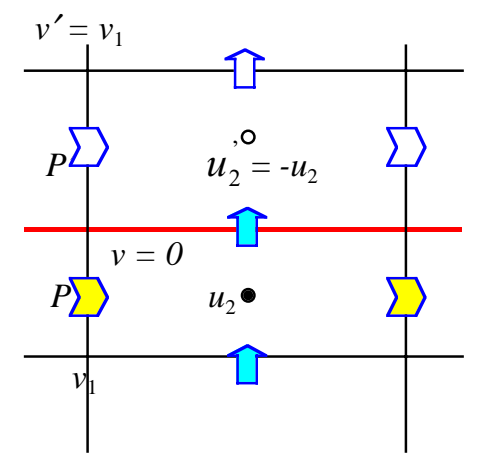

(b)

Figure 3. Boundary conditions around (a) vertical wall, and (b) horizontal wall. The red thick line represents no-flow boundary and outside it are the reflection grids. 


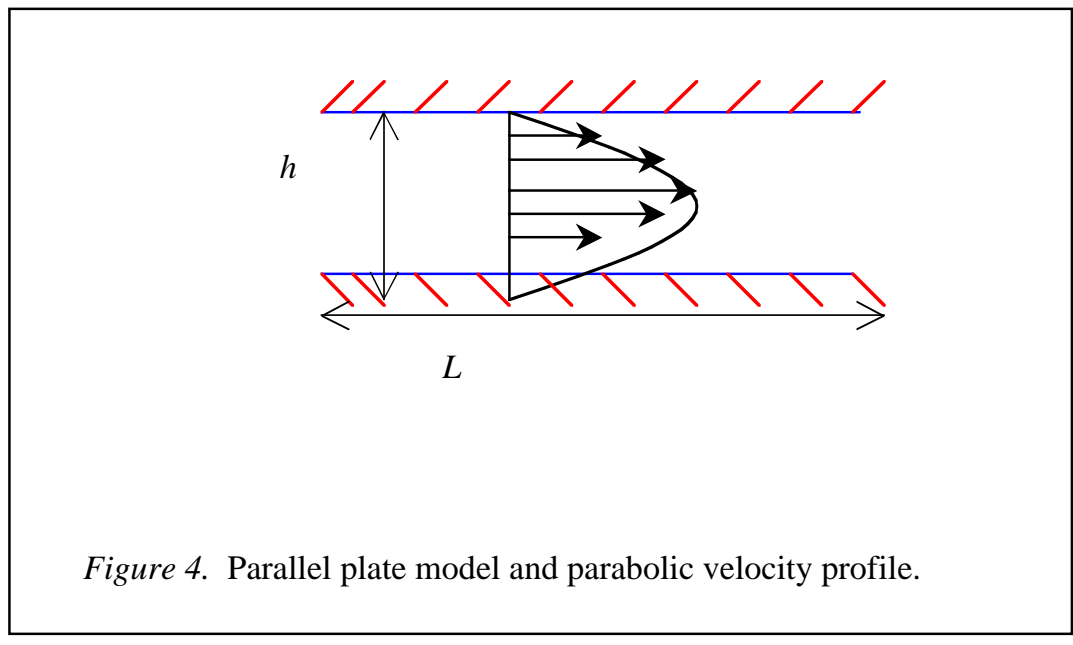




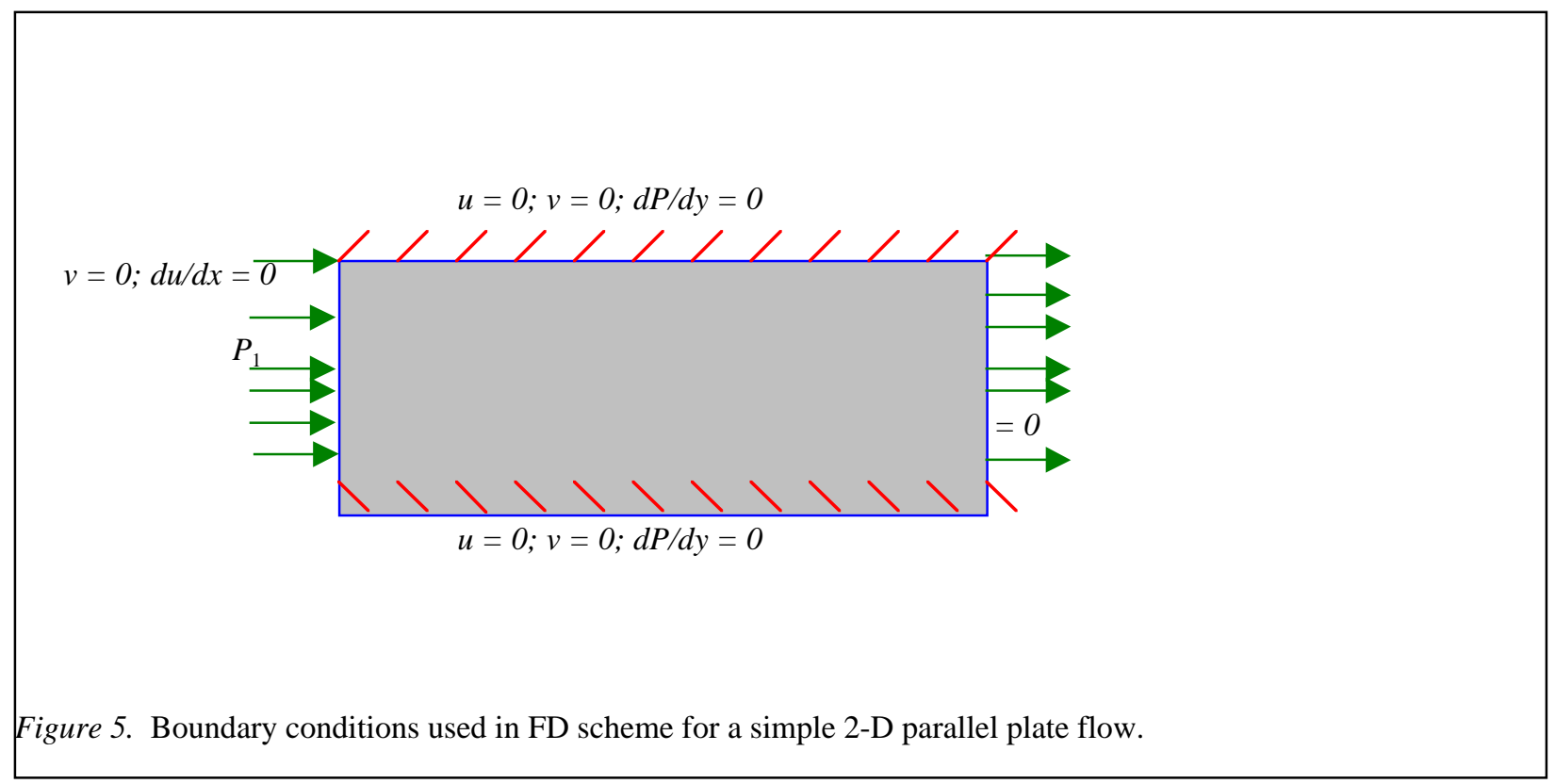




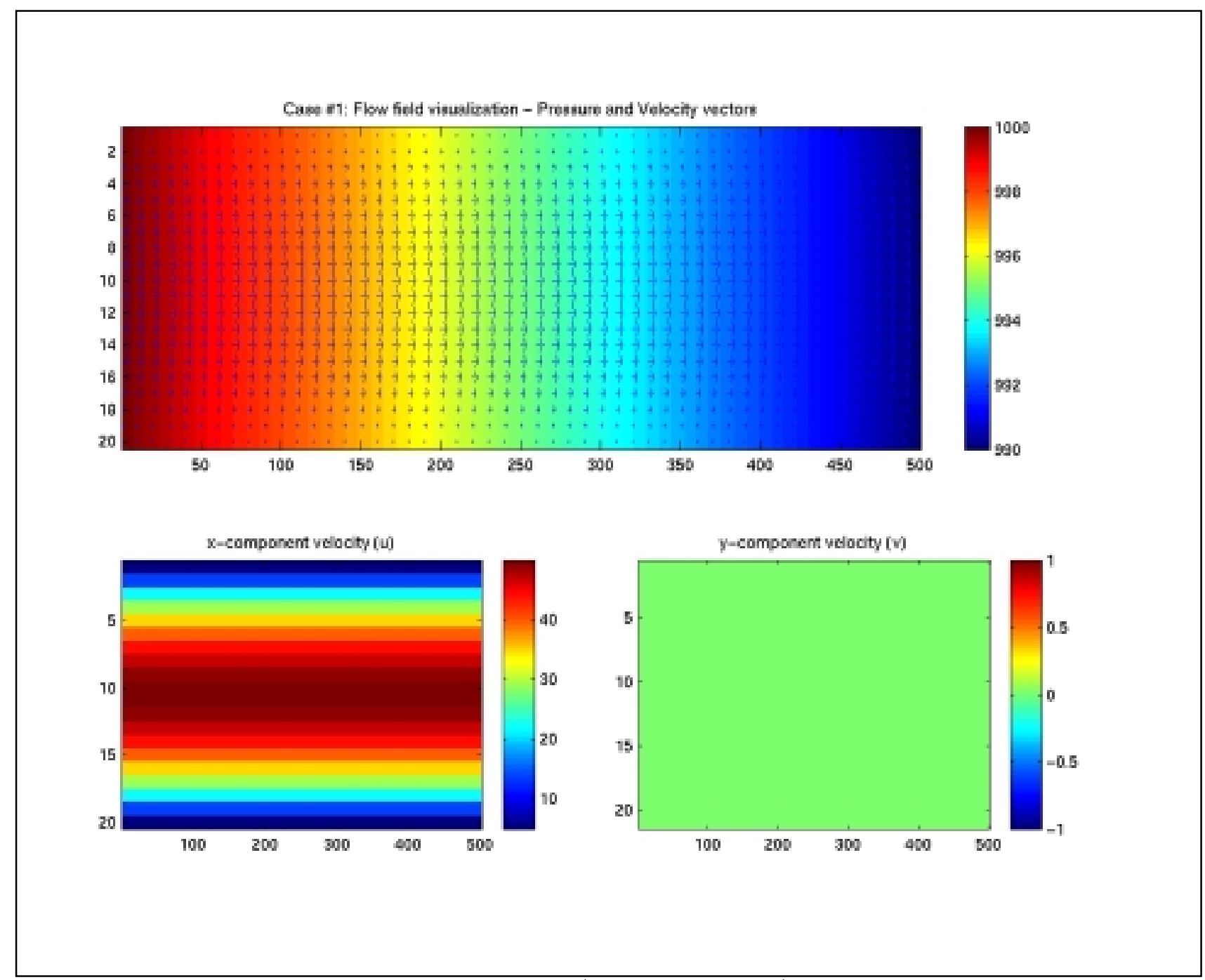

Figure 6. Flow simulation for case \#1. $\mathrm{X}=0 \rightarrow 500 \times 10^{-4} \mathrm{~m} ; \mathrm{Y}=0 \rightarrow 20 \times 10^{-4} \mathrm{~m}$. 

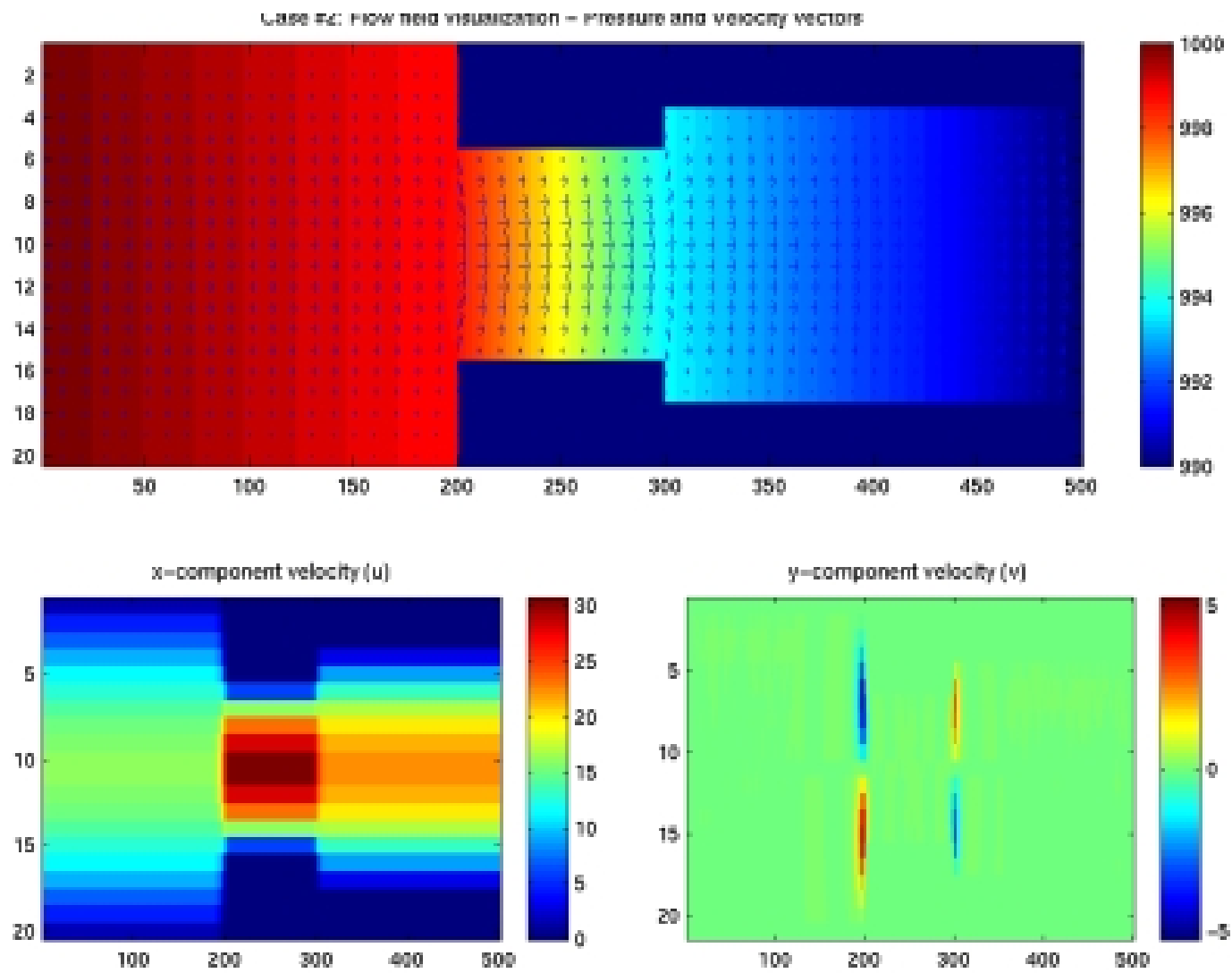

Figure 7. Flow simulation for case \#2. $\mathrm{X}=0 \rightarrow 500 \times 10^{-4} \mathrm{~m}$; $\mathrm{Y}=0 \rightarrow 20 \times 10^{-4} \mathrm{~m}$. 

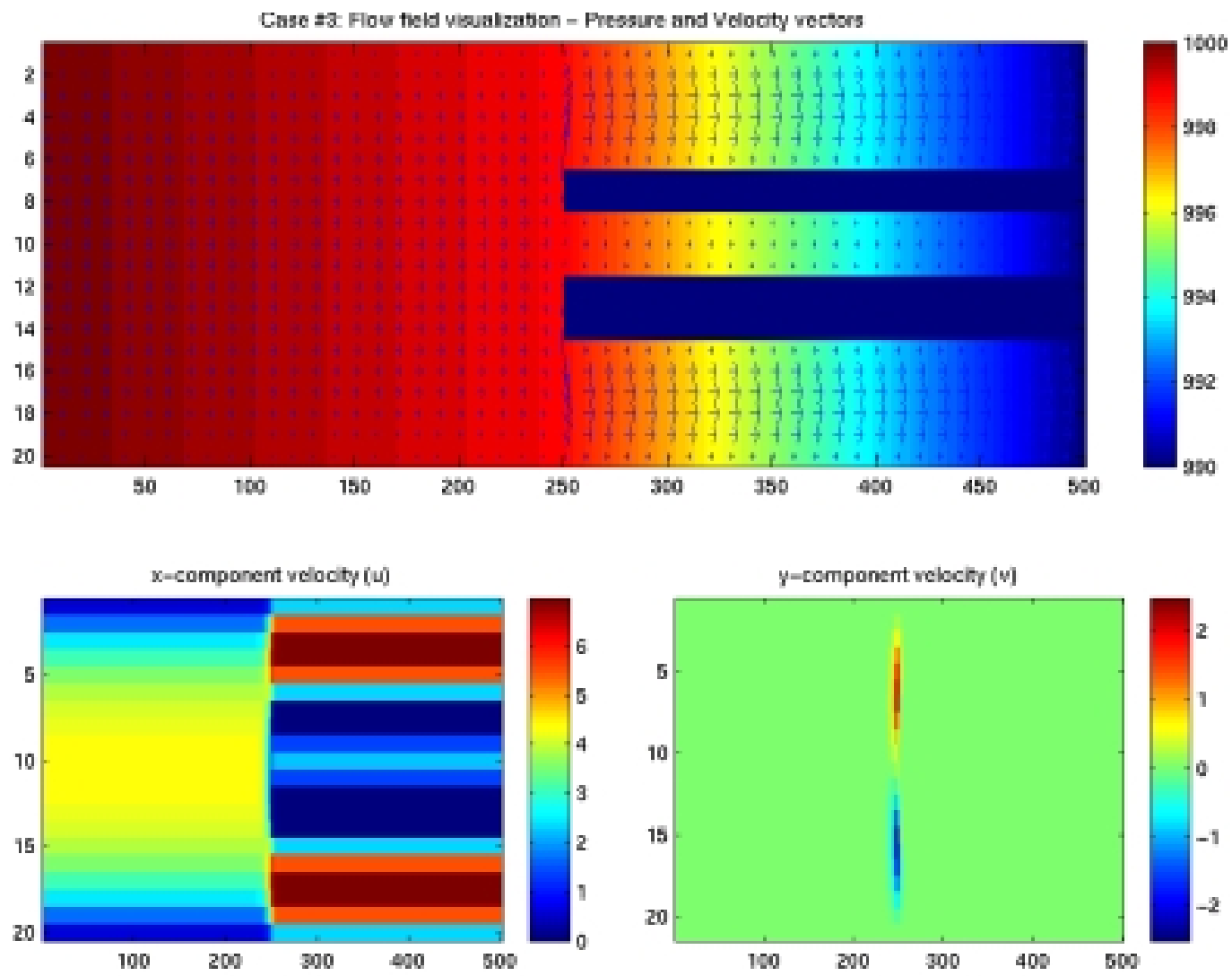

Figure 8. Flow simulation for case \#3. X $=0 \rightarrow 500 \times 10^{-4} \mathrm{~m} ; \mathrm{Y}=0 \rightarrow 20 \times 10^{-4} \mathrm{~m}$. 

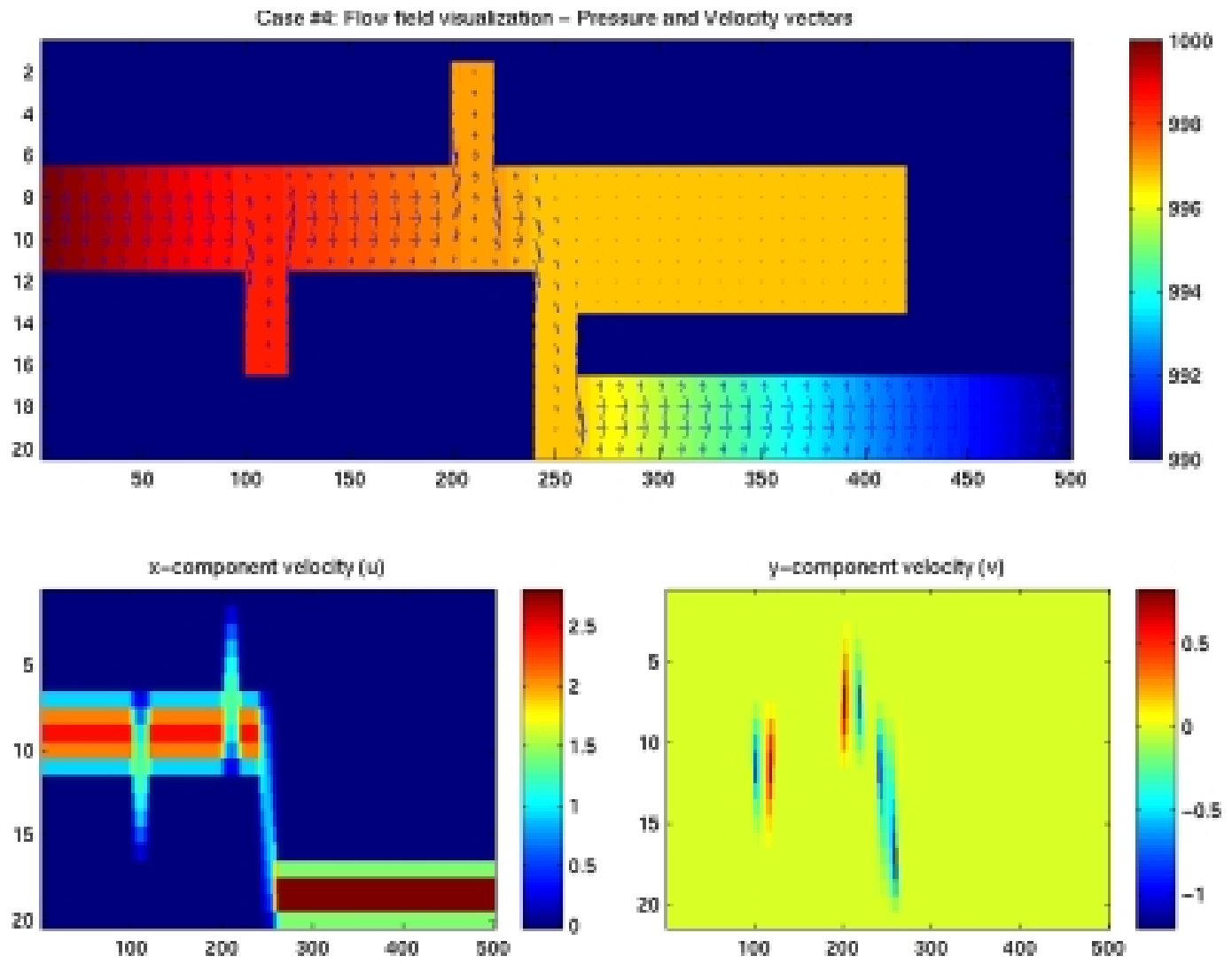

Figure 9. Flow simulation for case \#4. $\mathrm{X}=0 \rightarrow 500 \times 10^{-4} \mathrm{~m} ; \mathrm{Y}=0 \rightarrow 20 \times 10^{-4} \mathrm{~m}$. 

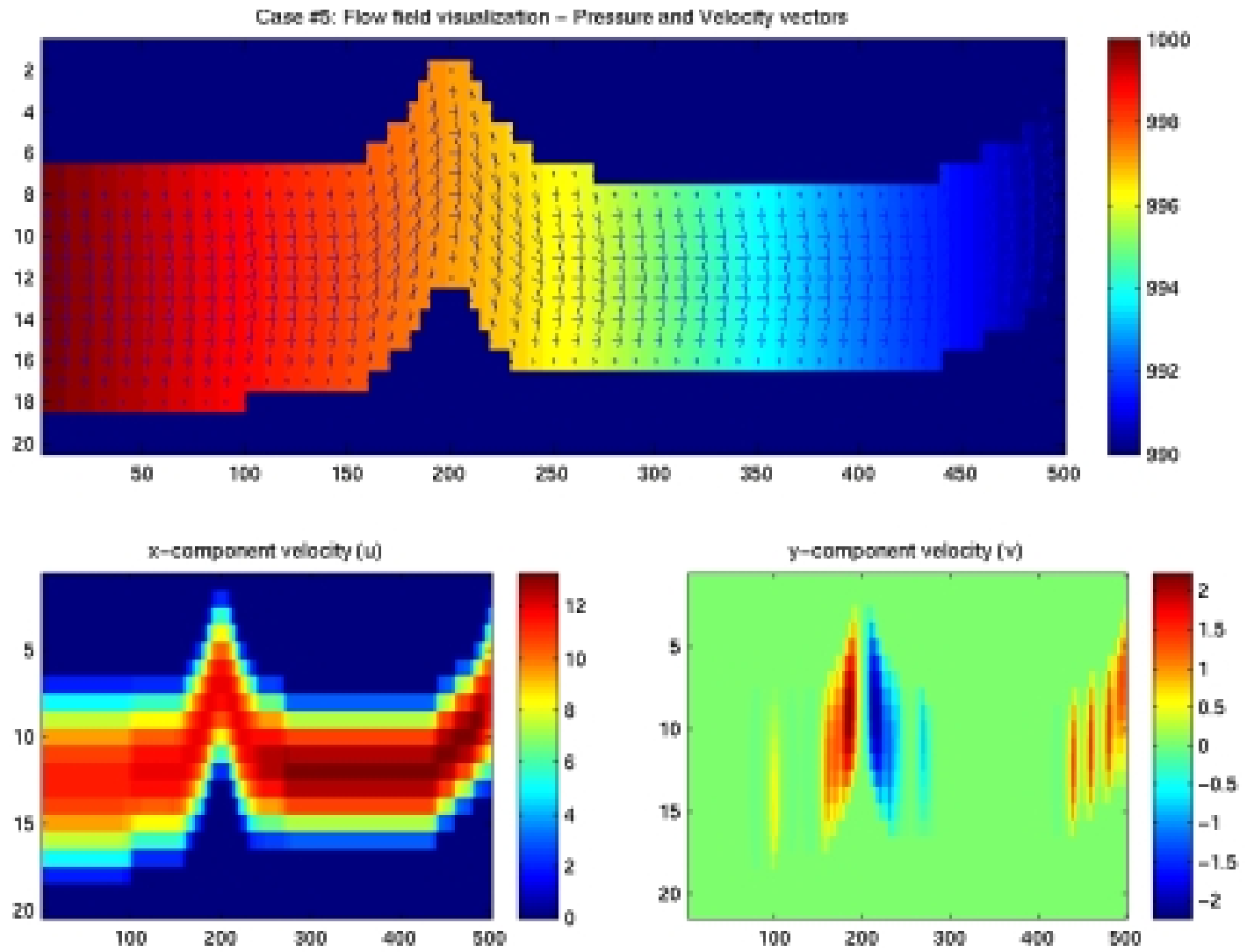

Figure 10. Flow simulation for case \#5. X $=0 \rightarrow 500 \times 10^{-4} \mathrm{~m} ; \mathrm{Y}=0 \rightarrow 20 \times 10^{-4} \mathrm{~m}$. 

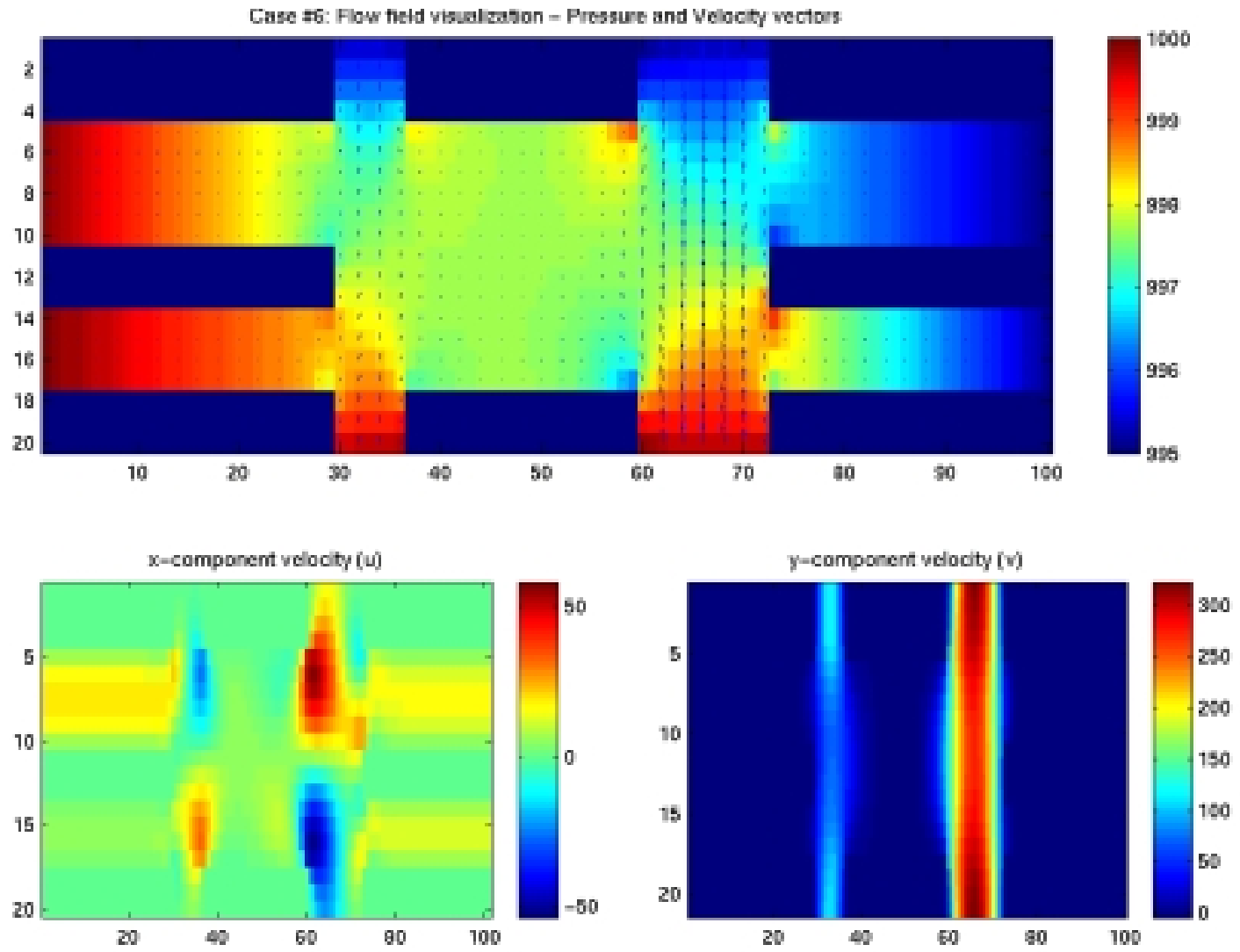

Figure 11. Flow simulation for case \#6. $\mathrm{X}=0 \rightarrow 100 \times 10^{-4} \mathrm{~m} ; \mathrm{Y}=0 \rightarrow 20 \times 10^{-4} \mathrm{~m}$. 


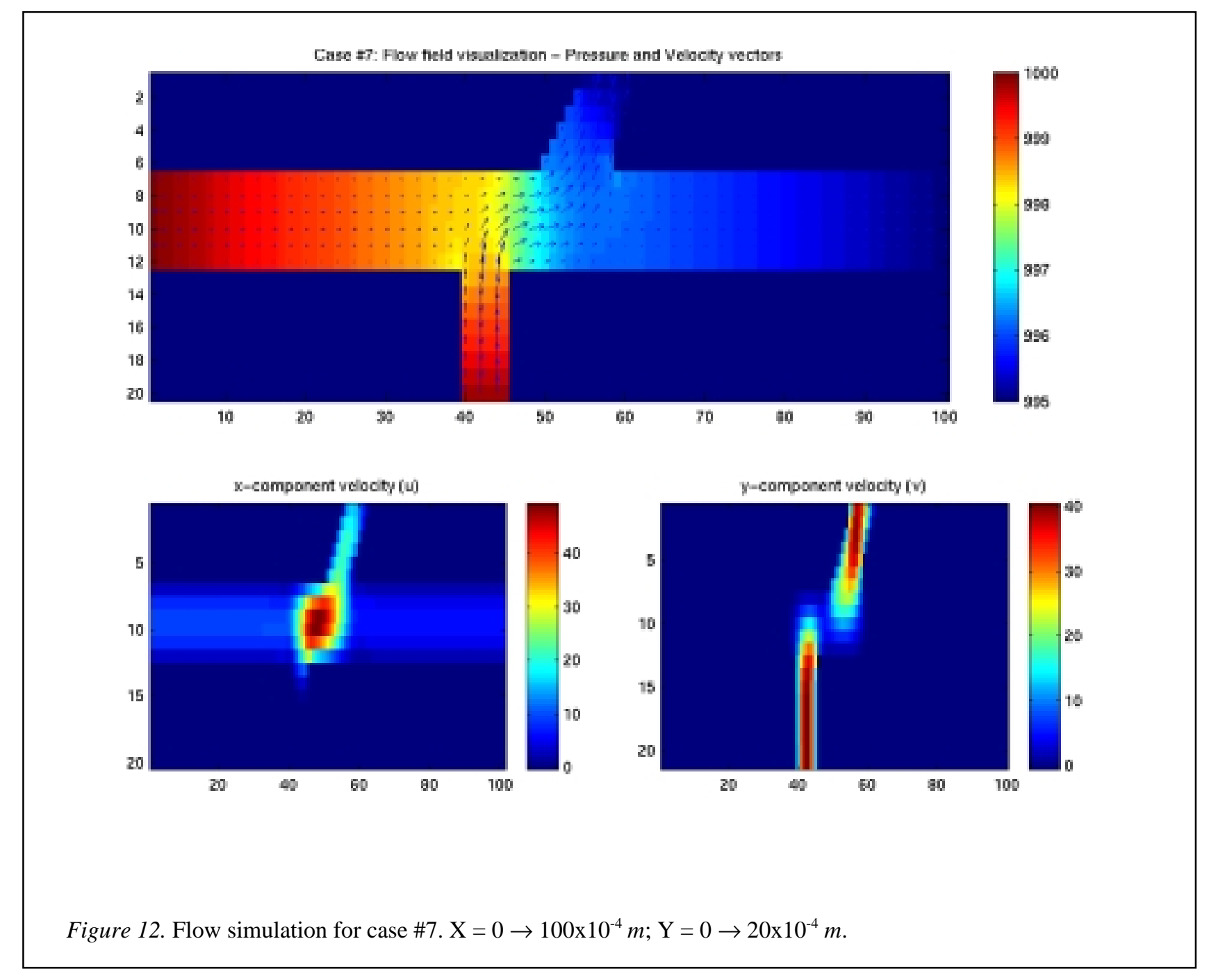



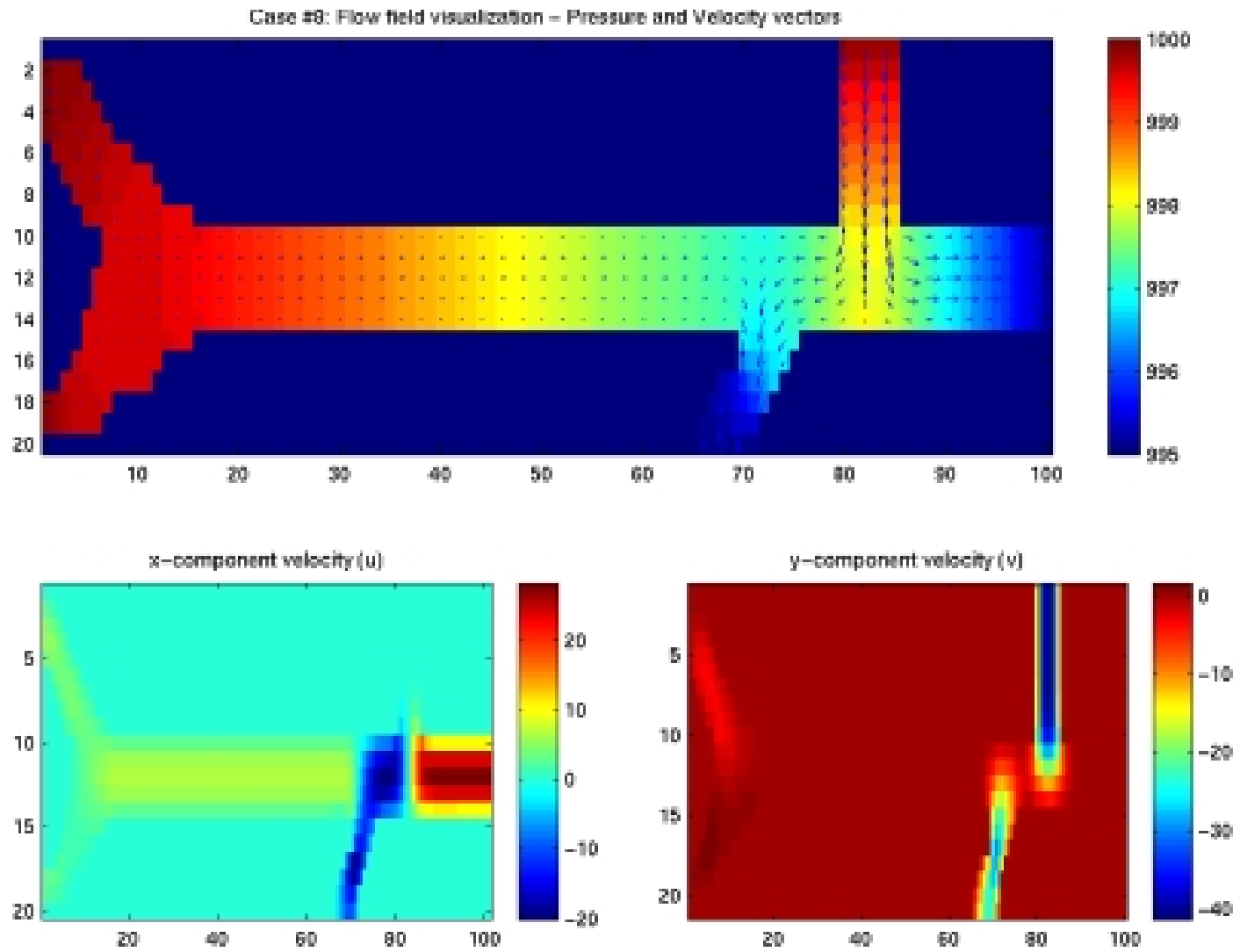

Figure 13. Flow simulation for case \#8. $\mathrm{X}=0 \rightarrow 100 \times 10^{-4} \mathrm{~m} ; \mathrm{Y}=0 \rightarrow 20 \times 10^{-4} \mathrm{~m}$. 

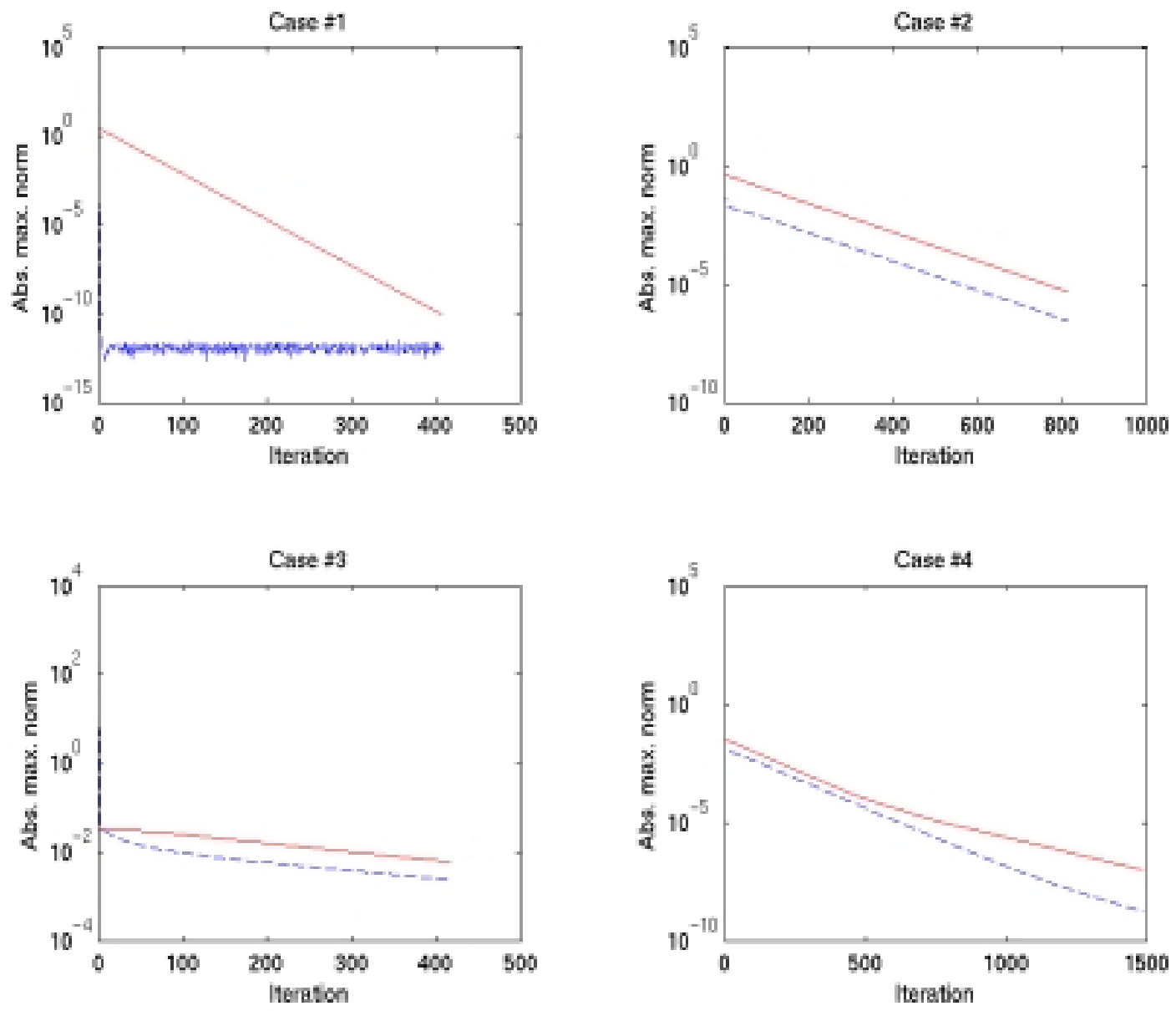

Figure 14. Maximum change of variables with iterations for cases \#1 - \#4. Solid red line indicates maximum velocity change and dashed blue line represents maximum pressure change. 

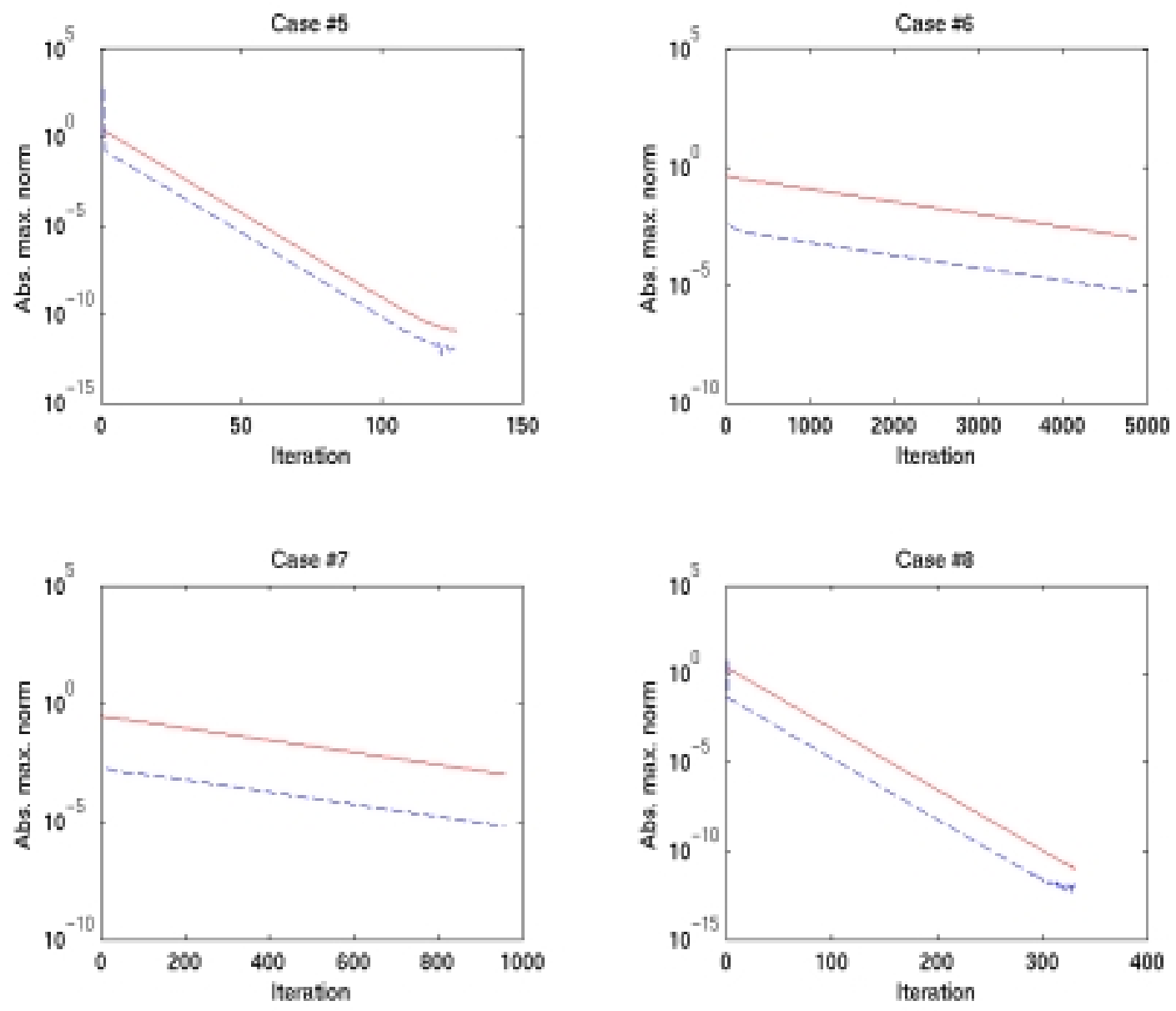

Figure 15. Maximum change of variables with iterations for cases \#4 - \#8. Solid red line indicates maximum velocity change and dashed blue line represents maximum pressure change. 
Table 1: Flow simulation summary

\begin{tabular}{|c|c|c|c|c|c|c|c|c|c|}
\hline $\begin{array}{l}\text { Run } \\
\text { specs }\end{array}$ & Case \# & 1 & 2 & 3 & 4 & 5 & 6 & 7 & 8 \\
\hline \multirow{2}{*}{$\begin{array}{l}a \\
\tilde{0} \\
\frac{a}{0} \\
\tilde{\Xi} \\
\tilde{a}\end{array}$} & $\begin{array}{l}\text { Max. length } \\
\left(10^{-4} \mathrm{~m}\right)\end{array}$ & 500 & 500 & 500 & 500 & 500 & 100 & 100 & 100 \\
\hline & $\begin{array}{l}\text { Max. aperture } \\
\left(10^{-4} \mathrm{~m}\right)\end{array}$ & 20 & 20 & 20 & 20 & 20 & 20 & 20 & 20 \\
\hline \multirow{3}{*}{ 全. } & $\Delta \mathrm{x}\left(10^{-4} \mathrm{~m}\right)$ & 1 & 1 & 1 & 1 & 1 & 1 & 1 & 1 \\
\hline & $\Delta \mathrm{y}\left(10^{-4} \mathrm{~m}\right)$ & 1 & 1 & 1 & 1 & 1 & 1 & 1 & 1 \\
\hline & $\Delta \mathrm{t}(\mathrm{sec})$. & 0.01 & 0.001 & 0.0001 & 0.0001 & 0.01 & 0.0001 & 0.0001 & 0.001 \\
\hline \multirow{3}{*}{ } & Criteria, $\varepsilon$ & $1 \times 10^{-10}$ & $5 \times 10^{-6}$ & $6 \times 10^{-3}$ & $1 \times 10^{-7}$ & $1 \times 10^{-11}$ & $1 \times 10^{-3}$ & $1 \times 10^{-3}$ & $1 \times 10^{-11}$ \\
\hline & $\begin{array}{l}\text { Total no. of } \\
\text { iterations }\end{array}$ & 407 & 814 & 417 & 1486 & 127 & 4875 & 958 & 332 \\
\hline & $\begin{array}{l}\text { Elapsed time } \\
\text { (sec.) }\end{array}$ & $3.69 \times 10^{4}$ & $2.45 \times 10^{4}$ & $2.02 \times 10^{4}$ & $1.48 \times 10^{4}$ & $1.5 \times 10^{3}$ & $5.64 \times 10^{4}$ & 410.85 & 252.24 \\
\hline \multirow{4}{*}{ 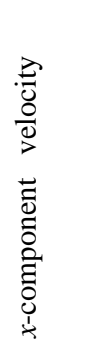 } & $\operatorname{Min} u(m / s)$ & 0.0 & -0.05 & 0.0 & -0.0201 & 0.0 & -53.7 & -0.3 & -20.14 \\
\hline & $\operatorname{Max} u(m / s)$ & 49.95 & 30.79 & 6.99 & 2.8102 & 12.3 & 58.25 & 49.42 & 28.33 \\
\hline & Mean $u(m / s)$ & 33.43 & 13.593 & 3.68 & 1.1116 & 8.28 & 6.3978 & 8.6027 & 3.5821 \\
\hline & $\operatorname{Var} u$ & 218.45 & 50.63 & 3.12 & 1.0055 & 13.37 & 159.26 & 86.4 & 69.17 \\
\hline \multirow{4}{*}{ 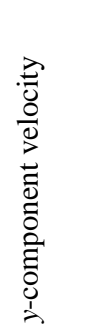 } & $\operatorname{Min} v(m / s)$ & 0.0 & -5.24 & -2.5 & -1.2040 & -2.04 & -4.835 & -0.5 & -41.31 \\
\hline & $\operatorname{Max} v(m / s)$ & 0.0 & 5.24 & 2.5 & 0.8272 & 2.18 & 322.60 & 40.49 & 1.55 \\
\hline & Mean $v(m / s)$ & 0.0 & 0 & 0 & -0.0182 & 0.07 & 49.9795 & 4.6405 & -3.9915 \\
\hline & $\operatorname{Var} v$ & 0.0 & 0.15 & 0.02 & 0.0166 & 0.2388 & 7872.11 & 103.69 & 87.05 \\
\hline \multirow{3}{*}{ 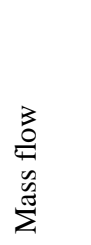 } & $\Sigma \mathrm{Q}_{\mathrm{in}}\left(10^{-4} \mathrm{~m}^{2} / \mathrm{s}\right)$ & 668.66 & 213.93 & 64.64 & 8.45 & 91.22 & 3494.65 & 211.51 & 195.83 \\
\hline & $\Sigma \mathrm{Q}_{\text {out }}\left(10^{-4} \mathrm{~m}^{2} / \mathrm{s}\right)$ & 668.66 & 212.31 & 64.10 & 8.41 & 91.76 & 3494.45 & 212.01 & 195.67 \\
\hline & $\%$ Error & 0.0 & 0.76 & 0.84 & 0.47 & 0.59 & 0.0057 & 0.24 & 0.08 \\
\hline \multirow{2}{*}{ 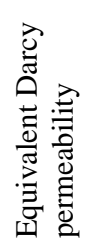 } & $\mathrm{k}_{\mathrm{x}}(\mathrm{d})$ & 338729.5 & 137731.1 & 37287.6 & 11263.3 & 83897.1 & 25930.3 & 34866.7 & 14518.3 \\
\hline & $\mathrm{k}_{\mathrm{y}}(\mathrm{d})$ & --- & --- & --- & --- & --- & 40513.4 & 3761.6 & $3235.5 \downarrow$ \\
\hline
\end{tabular}

